\title{
CHRONIC EXPOSURE TO ARSENITE ENHANCES INFLUENZA VIRUS INFECTION IN CULTURED CELLS
}

\author{
by \\ Eva Amouzougan \\ Copyright $\odot$ Eva Amouzougan 2019 \\ A Dissertation Submitted to the Faculty of the \\ DEPARTMENT OF PHARMACOLOGY AND TOXICOLOGY \\ In Partial Fulfillment of the Requirements \\ For the Degree of \\ DOCTOR OF PHILOSOPHY \\ In the Graduate College \\ THE UNIVERSITY OF ARIZONA
}

2019 


\section{THE UNIVERSITY OF ARIZONA}

\section{GRADUATE COLLEGE}

As members of the Dissertation Committee, we certify that we have read the dissertation prepared by Eva Amouzougan, titled Chronic Exposure to Arsenite Enhances Influenza Virus Infection in Cultured Cells and recommend that it be accepted as fulfilling the dissertation requirement for the Degree of Doctor of Philosophy.

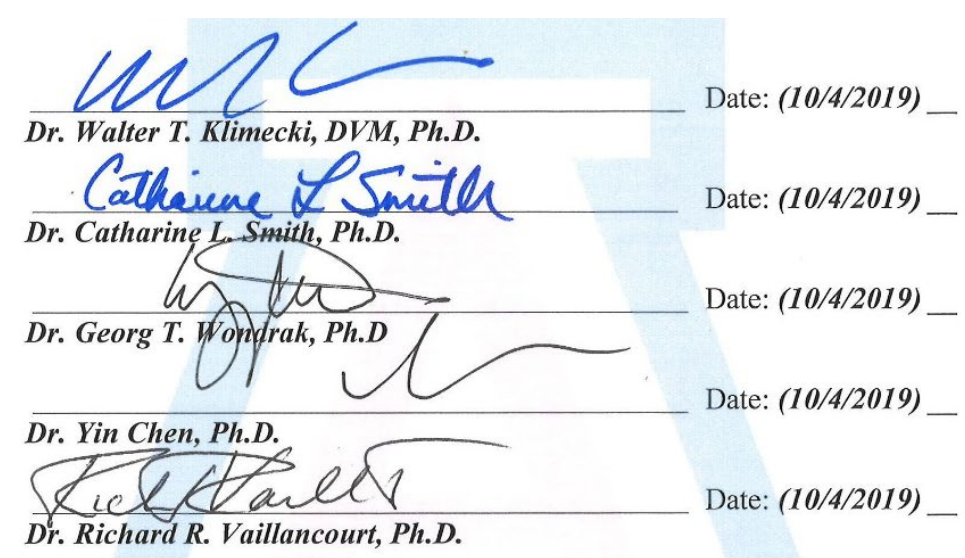

Final approval and acceptance of this dissertation is contingent upon the candidate's submission of the final copies of the dissertation to the Graduate College.

I hereby certify that I have read this dissertation prepared under my direction and recommend that it be accepted as fulfilling the dissertation requirement.

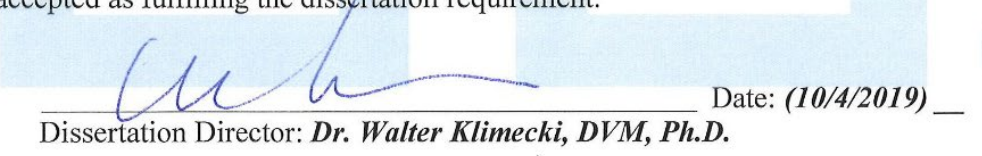




\section{STATEMENT BY AUTHOR}

This dissertation has been submitted in partial fulfillment of the requirements for an advanced degree at the University of Arizona and is deposited in the University Library to be made available to borrowers under rules of the Library.

Brief quotations from this dissertation are allowable without special permission, provided that an accurate acknowledgement of the source is made. Requests for permission for extended quotation from or reproduction of this manuscript in whole or in part may be granted by the head of the major department or the Dean of the Graduate College when in his or her judgment the proposed use of the material is in the interests of scholarship. In all other instances, however, permission must be obtained from the author.

SIGNED: Eva Amouzougan 


\section{ACKNOWLEDGMENT}

I would like to thank my mentor, Dr. Walter Klimecki for his mentorship, guidance, knowledge, expertise, encouragement, leadership, and the support he has provided me during my time in the program. Thank you for pushing me towards excellence and for turning me into the independent scientist that I am today. I am forever grateful for having an amazing mentor that took the time to mentor me and provided me with the resources I needed to succeed.

I would like to thank all my committee members, Dr. Catherine Smith, Dr. Georg Wondrak, Dr. Yin Chen, and Dr. Richard Vaillancourt for their expertise, guidance, advice, knowledge, and support. I am so grateful and honored to have my work evaluated by highly esteemed scientists. I am appreciative of all the time you took to evaluate my work and give constructive feedback to improve.

I would like to thank all the past members of the Klimecki's laboratory. Especially; Dr. Scott Malm, Jason Town, Anoop Hunjan, and Ricky Lira for all their support during my time in Klimecki's laboratory. I am thankful for the students, staff members, and faculties in the Department of Pharmacology and Toxicology. Next, I would like to acknowledge Sonya Basurto for being so wonderful to me during my time in the program. Thank you for your help, encouragement, wisdom, and constant support during my time in the program. I will always be grateful to you. Lastly, a thank you to Jo Eversole for all her support. Thank you for always making sure that I have such an amazing selection of my favorite teas in the office to keep me going. 
To my family, thank you for all your love, support, and encouragement throughout this journey. I especially would like to thank my parents William and Ayina Amouzougan for always being there for me and encouraging me to stay strong during hard times. It is your love and enthusiasm that has carried me through to the end. I am grateful for my amazing siblings who have supported me throughout this journey. Thank you for motivating me to pursue my dreams and for giving me a reason to keep going.

Additionally, a very special thank you to Kevin Haight for always being there for me throughout this journey. Your love, wisdom, and support helped me through this challenging journey. I am forever grateful for your kind soul and truly blessed to have you in my life. I would also like to thank Dr. Benjamin Katchman for being an amazing support system in my life and for making this journey enjoyable. I am lucky to have such an amazing person in my life. And finally, I would like to thank Julia Cheng, Dr. Anika Dzierlenga, Dr. Jessica Sapiro, Dr. Usir Younis, and Dr. Shayesteh Ferdosi for supporting me and making this journey fun. 


\section{DEDICATION}

I would like to dedicate my dissertation work to my lovely and humble parents, William Semenou Amouzougan and Ayina Amouzougan, who have sacrificed everything so that my siblings and I could have better opportunities in life. There are no words to express how grateful I am to have your love and support throughout this journey. 
1 Contents

CHRONIC EXPOSURE TO ARSENITE ENHANCES INFLUENZA VIRUS INFECTION IN CULTURED CELLS.................................. 1

LIST OF TABLES

LIST OF FIGURES

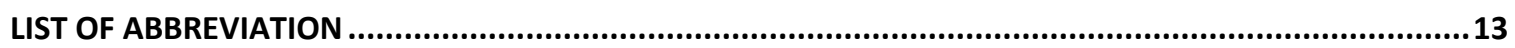

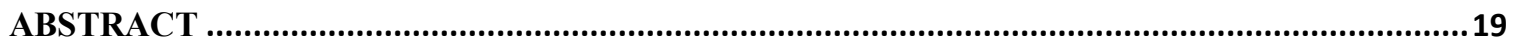

1. CHAPTER 1: INTRODUCTION, RESEARCH QUESTION, AND SPECIFIC AIMS .............21

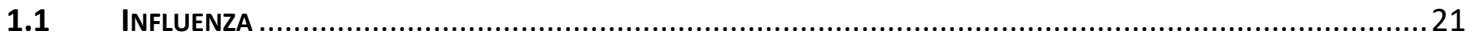

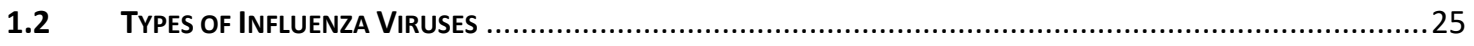

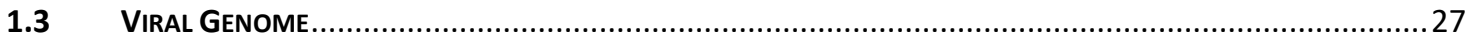

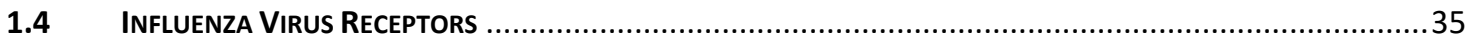

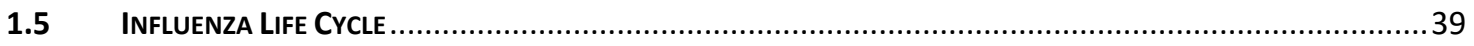

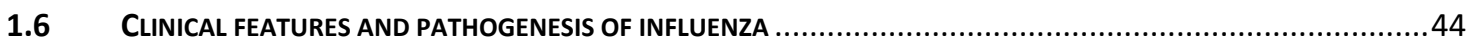

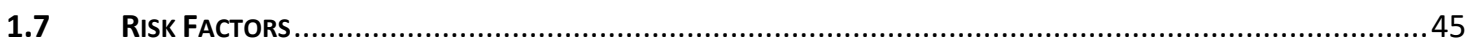

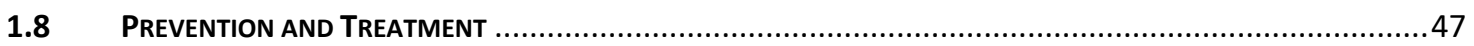

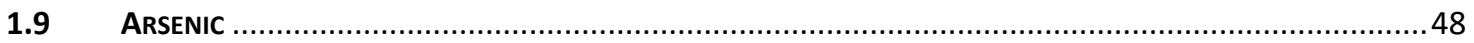

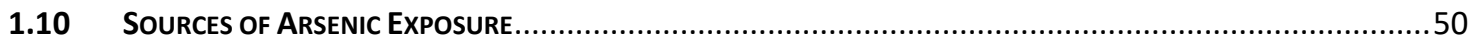

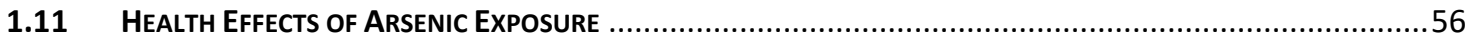

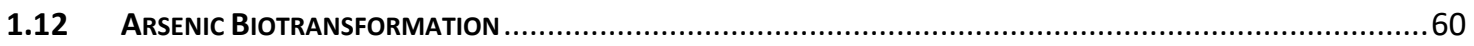

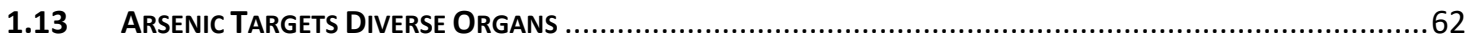

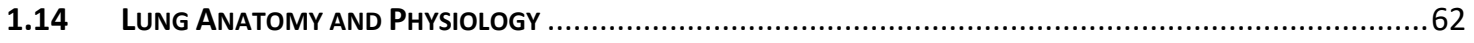

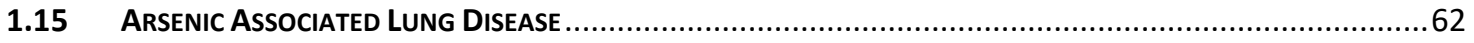

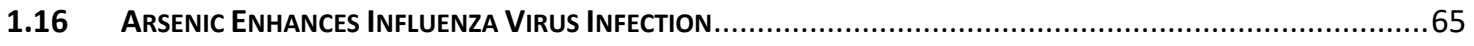




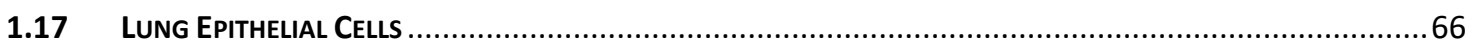

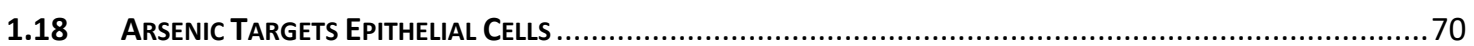

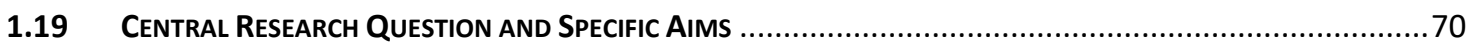

2 CHAPTER 2: CHRONIC EXPOSURE TO ARSENITE ENHANCES INFLUENZA VIRUS INFECTION IN

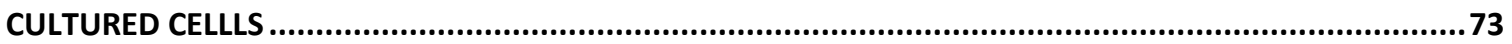

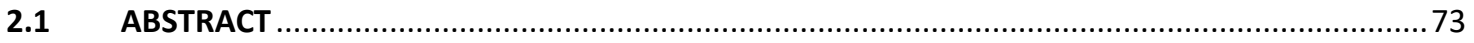

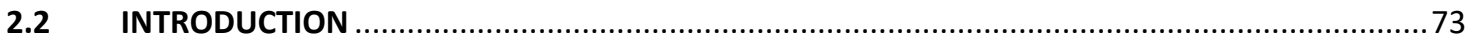

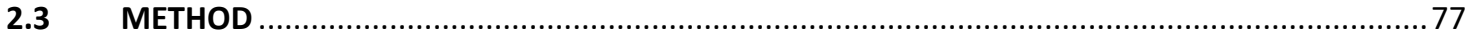

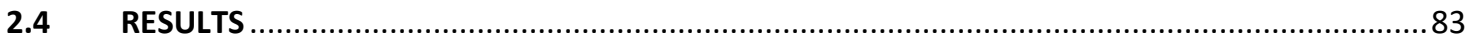

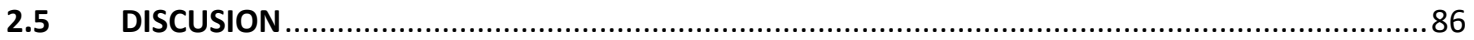

3 CHAPTER 3: CHRONIC ARSENITE EXPOSURE ENHANCES INFLUENZA VIRUS INFECTION IN HUMAN

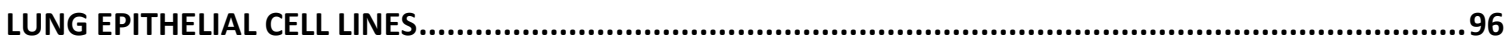

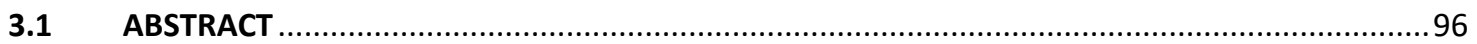

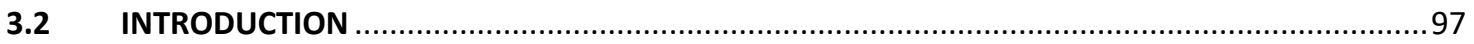

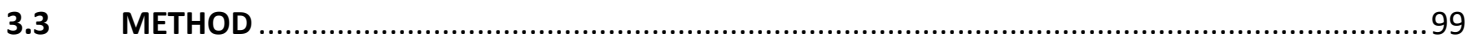

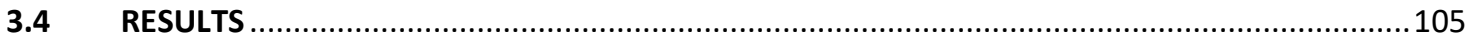

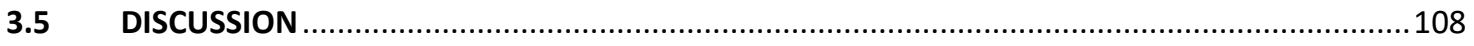

4 APPENDIX A: ARSENITE EXPOSURE MEDIATES INHIBITION OF PI3K/AKT SIGNALING PATHWAY IN

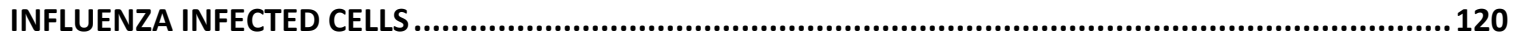

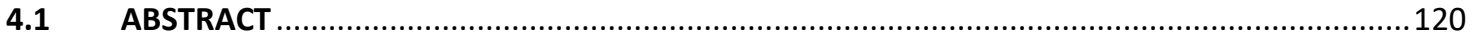

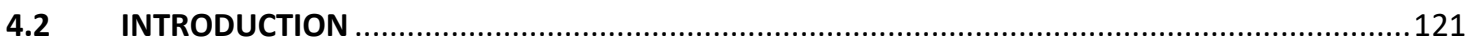

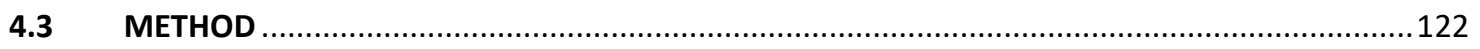

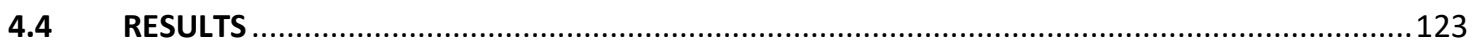

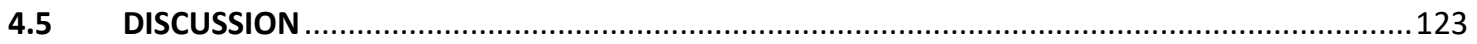


5 APPENDIX B: ARSENITE EXPOSURE INHIBITS TRIM22 AND MX1 PROTEINS EXPRESSION IN BEAS-2B

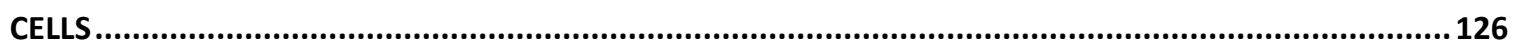

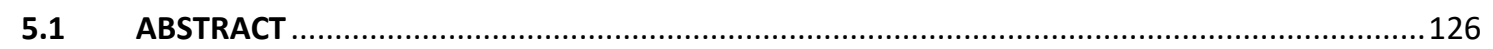

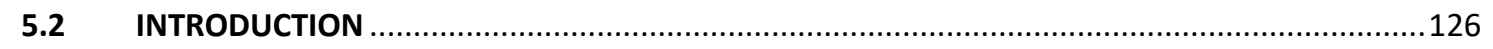

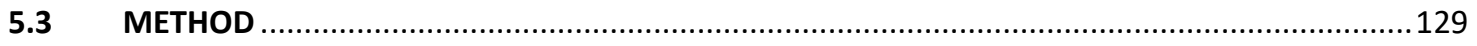

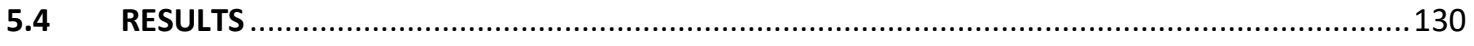

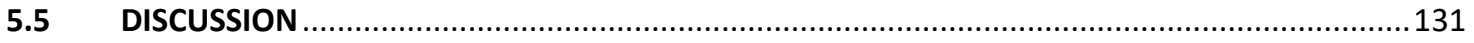

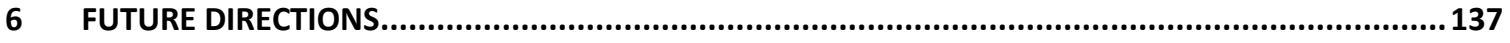

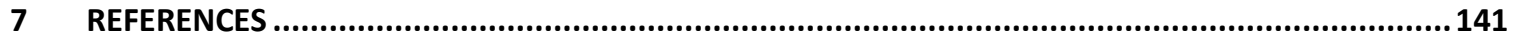




\section{LIST OF TABLES}

Table 1: List of influenza pandemics from the past one hundred years (Saunders-Hastings and

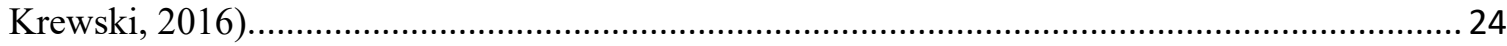




\section{LIST OF FIGURES}

Figure 1: Cartoon representation of influenza $C$ and $B / A$ virus particles........................... 33

Figure 2: Cartoon representation of influenza D virus particles........................................ 34

Figure 3: Types of sialic acids and glycosidic bonds.................................................... 38

Figure 4: Cartoon representative of influenza A virus structure and influenza virus life

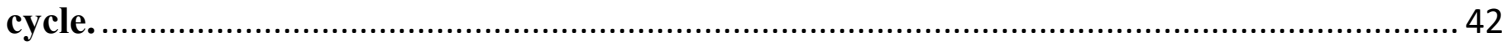

Figure 5: Inorganic arsenic biotransformation pathway showing the reduction of arsenate to arsenite and oxidative methylation to pentavalent and trivalent forms.

Figure 6: Cartoon representation of the epithelial cells acting as a barrier against

respiratory viruses.

Figure 7: Exposure to sodium arsenite enhanced influenza A/WSN/33 (H1N1) virus

infection in MDCK cells 91

Figure 8: Sodium arsenite exposure leads to increased plaque formation in MDCK cells at 48h p.i with influenza A/WSN/33 (H1N1) virus.

Figure 9: Sodium arsenite reduced the efficacy of anti-viral drug oseltamivir.

Figure 10: Exposure to sodium arsenite enhanced influenza A/WSN/33 (H1N1) virus attachment in MDCK cells. 94

Figure 11: Exposure to sodium arsenite increases $\alpha-2,3$-linked sialic acid quantity in MDCK cells.

Figure 12 : Sodium arsenite exposure leads to increased plaque formation in MDCK cells at 48h p.i with influenza A/WSN/33 (H1N1) virus. 114

Figure 13: Exposure to sodium arsenite enhanced influenza A/Udorn/72 (H3N2) virus infection in BEAS-2B cells 
Figure 14: Exposure to sodium arsenite enhanced influenza A/Udorn/72 (H3N2) virus infection in $\mathbf{A 5 4 9}$ cells. .

Figure 15: Exposure to sodium arsenite enhanced influenza A/Udorn/72 (H3N2) virus attachment in BEAS-2B cells.

Figure 16: Exposure to sodium arsenite increases $\alpha-2,3-$ linked sialic acid quantity in BEAS$2 B$ cells 118

Figure 17: $\alpha-2,6-$ linked sialic acid quantity in BEAS-2B cells 119

Figure 18 : Exposure to sodium arsenite correlates with decreased phospho-Akt expression in MDCK cells 125 Figure 19: Exposure to sodium arsenite correlates with decreased TBK1 and Mx1 protein expression in BEAS-2B cells 134

Figure 20: Exposure to sodium arsenite correlates with decreased TRIM22 mRNA quantity BEAS-2B cells. 135 


\section{LIST OF ABBREVIATION}

\begin{tabular}{|c|c|}
\hline ALRI & acute lower respiratory infection \\
\hline AS & arsenic \\
\hline AS3MT & arsenicmethyltransferase \\
\hline ATF4 & activation transcription factor 2 \\
\hline BEAS-2B & bronchial epithelial cell \\
\hline BM2 & M2 protein of influenza B \\
\hline CBER & center for biologics evaluation and research \\
\hline $\mathrm{Cd}$ & cadmium \\
\hline $\mathrm{CHOP}$ & C/EBP homologous protein \\
\hline CLR & c-type lectin receptor \\
\hline CM2 & $\mathrm{M} 2$ protein of influenza $\mathrm{C}$ \\
\hline COPD & obstructive pulmonary disease \\
\hline cRNA & complementary ribonucleic acid \\
\hline Cy5 & cyanine-5 \\
\hline $\mathrm{DC}$ & dendritic cells \\
\hline DEP & diesel exhaust particles \\
\hline DM & diabetes mellitus \\
\hline $\mathrm{DMA}^{\mathrm{III}}$ & dimethylarsinous acid \\
\hline $\mathrm{DMA}^{\mathrm{V}}$ & dimethyl arsenic acid \\
\hline DM1 & M1 protein of influenza D \\
\hline DM2 & M2 protein of influenza D \\
\hline DPHE & department of public health and engineering \\
\hline
\end{tabular}




\begin{tabular}{ll} 
ECMV & encephalomyocarditis virus \\
EDTA & ethylenediaminetetraacetic acid \\
eIF2 $\alpha$ & eukaryotic translocation initiation factor 2 subunit $\alpha$ \\
EGFR & epidermal growth factor receptor \\
EMT & epithelial-to-mesenchymal transition \\
EPA & environmental protection agency \\
ER & endoplasmic reticulum \\
EU & European union \\
FBS & fetal bovine serum \\
FDA & food and drug administration \\
FEV & forced expiratory volume \\
FITC & fluorescein isothiocyanate \\
FVC & forced vital capacity \\
GAPDH & glyceraldehyde 3-phosphate dehydrogenase \\
GSH & glutathione \\
HA & haemagglutinin \\
HBE & human airway epithelial cells \\
HBV & hepatis B virus \\
HEF & hemagglutinin esterase-fusion \\
HO-1 & heme oxygenase-1 \\
HIV-1 & human immunodeficiency virus 1 \\
IAV & influenza A virus \\
ICV & influenza C virus \\
\hline
\end{tabular}




\begin{tabular}{|c|c|}
\hline IDV & influenza $\mathrm{D}$ virus \\
\hline IFN & interferon \\
\hline IFN- $\alpha$ & interferon-alpha \\
\hline IFN- $\beta$ & interferon-beta \\
\hline IFN- $\gamma$ & interferon-gamma \\
\hline IFN- $\lambda$ & lambda interferon \\
\hline IIV & inactivated influenza vaccine \\
\hline IL-2 & interlurkin-2 \\
\hline IL-6 & interlurkin-6 \\
\hline IRF & interferon regulatory factor \\
\hline IRF-3 & interferon regulatory factor-3 \\
\hline IRF-7 & interferon regulatory factor-7 \\
\hline ISG & interferon-stimulated gene \\
\hline ISG15 & interferon-stimulated gene 15 \\
\hline JAK & janus kinase \\
\hline JAK/STAT & janus kinase signal transducers \\
\hline JNK & c-Jun N-terminal protein kinase \\
\hline LAIV & live attenuated influenza vaccine \\
\hline M1 & matrix protein 1 \\
\hline M2 & matrix protein 2 \\
\hline MALII & maakia amuresis \\
\hline MCL & maximum contaminant level \\
\hline MCP-1 & monocyte chemoattractant protein- \\
\hline
\end{tabular}




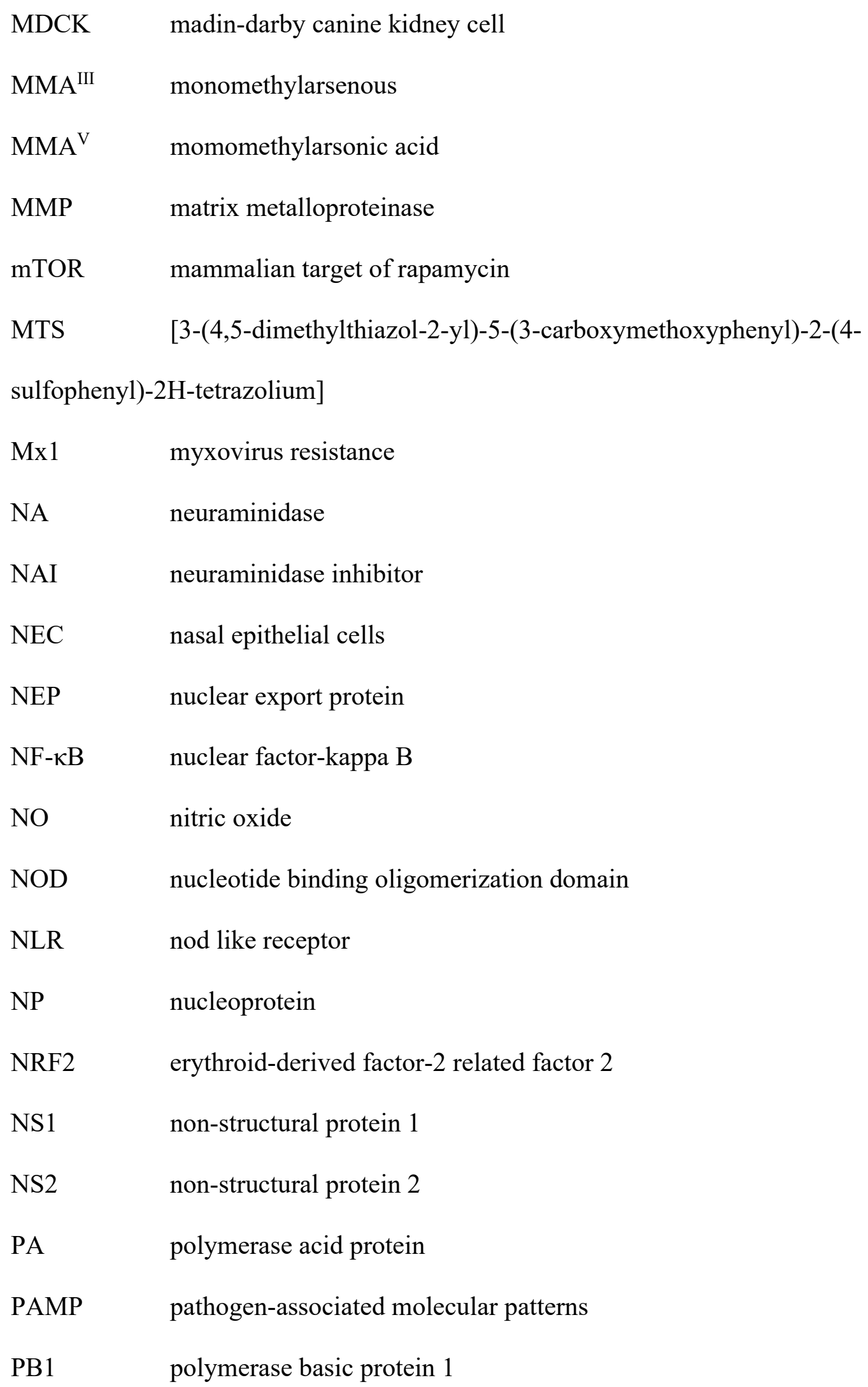




\begin{tabular}{|c|c|}
\hline PB1-F2 & proapoptotic peptide \\
\hline PB2 & polymerase basic protein 2 \\
\hline PBS & phosphate-buffer saline \\
\hline PERK & protein kinase-like ER kinase \\
\hline $\mathrm{PI} 3 \mathrm{~K} / \mathrm{Akt}$ & phosphoinositide-3-kinase B/Akt \\
\hline PPR & pattern recognition receptors \\
\hline RIV & recombinant influenza vaccine \\
\hline RLR & retinoic acid-inducible gene-I-like receptor \\
\hline RNP & ribonucleoprotein \\
\hline RPLPO & large ribosomal protein \\
\hline ROS & reactive oxygen species \\
\hline SNA & sambucus nigra \\
\hline UNICEF & united nation children fun \\
\hline VLP & virus like particle \\
\hline vRNA & virus ribonucleic acid \\
\hline vRNP & virus ribonucleoprotein \\
\hline VSMC & vascular smooth muscle cells \\
\hline SAM & s-adenosylmethione \\
\hline SDS-PAGE & sodium dodecyl sulfate polyacrylamide gel electrophoresis \\
\hline SOES & school of environmental studies \\
\hline TBK1 & tank-binding kinase \\
\hline $\mathrm{T}_{\mathrm{h}} 1$ & type 1 helper T cells \\
\hline $\mathrm{T}_{\mathrm{h}} 2$ & type 2 helper T cells \\
\hline
\end{tabular}


TIMP-1 tissue inhibitors of metalloproteinase

TLR toll like receptors

TRIM tripartite motif

WHO world health organization 


\begin{abstract}
Arsenic is a ubiquitous environmental toxicant that has been associated with various human diseases. Chronic, low dose arsenic exposure has been linked to greater morbidity from cancer, cardiovascular diseases, diabetes mellitus, neurological diseases, skin lesions (keratosis), and respiratory diseases. The primary route of arsenic exposure in humans is through inhalation and ingestion. It has been reported that millions of people are exposed to arsenic in drinking water worldwide. Recent epidemiological and experimental studies show that arsenic exposure is associated with enhanced influenza virus infection in vivo. The respiratory epithelium, which is the primary target of infection, plays an important role in innate immune defense by serving as a barrier against infection. Arsenic exposure has been shown to alter the epithelial barrier function by disrupting tight junction formation. Despite the strong link between arsenic exposure and respiratory infection, there are no reports investigating the effects of arsenic exposure on influenza virus infection in vitro. Therefore, we hypothesized that the effect of arsenic exposure on epithelial cells could enhance influenza virus infection.
\end{abstract}

In this study we characterized influenza A virus A/WSN/33 (H1N1) infection in arseniteexposed Madin-Darby Canine Kidney (MDCK) epithelial cells. We also characterized influenza A virus A/Udorn/72(H3N2) infection in human bronchial epithelial cells (BEAS2B), and adenocarcinomic human alveolar basal epithelial cells (A549) in order to uncover novel molecular mechanisms mediating arsenic enhanced influenza virus infection in vitro. 
We found that chronic exposure to sodium arsenite enhanced influenza A virus A/WSN/33 (H1N1) infection in MDCK cells. We observed increased viral matrix (M2) protein expression, increased viral mRNA quantity, and increased plaque area in the arseniteexposed MDCK cells. Similarly, we also found that chronic arsenite exposure enhanced influenza A virus A/Udorn/72 (H3N2) infection in BEAS-2B and A549 cells. We demonstrated that arsenite exposure resulted in significantly reduced cell viability and increased cytotoxicity in virus infected BEAS-2B and A549 cells.

The first step of influenza virus infection is the binding of the virus to the host surface receptors. Therefore, to determine the molecular mechanisms mediating arsenic-enhanced influenza virus infection in vitro, we quantified the amount of $\alpha-2,3$-linked and $\alpha-2,6-$ linked sialic acids-containing receptors in MDCK cells and BEAS-2B cells. We found that exposure to sodium arsenite resulted in increased $\alpha$-2,3-linked sialic acid expression, leading to increased virus attachment and enhanced infection in MDCK cells. On the contrary, we did not observe a significant change in $\alpha-2,3-$ linked sialic acid expression in BEAS-2B cells.

In conclusion, these studies show that exposure to sodium arsenite enhances influenza virus infection in vitro. This is consistent with previous reports showing that arsenite exposure enhances influenza virus infection in vivo. Furthermore, we uncover novel potential molecular mechanisms mediating arsenic-enhanced infection in vitro. 


\section{CHAPTER 1: INTRODUCTION, RESEARCH QUESTION, AND SPECIFIC AIMS}

\subsection{Influenza}

Influenza virus has been around since ancient times. Historical data suggests that influenza was first described by Hippocrates in 412 BC (Barberis, Myles, Ault, Bragazzi, \& Martini, 2016; Pappas, Kiriaze, \& Falagas, 2008; Saunders-Hastings \& Krewski, 2016). The first influenza epidemic can be traced back to the 12th century (Barberis et al., 2016; Potter, 2001). In 1933, a team of scientists by the names of Wilson Smith, Christopher H. Andrewes, and Patrick Laidlaw successfully isolated influenza virus from patients (Bresalier, 2012; Virus, 1938). Influenza, also known as the flu, is an important respiratory pathogen that causes infectious diseases in humans and animals (Hayden et al., 2000; Taubenberger \& Morens, 2008; Webster \& Govorkova, 2014). It causes mild to severe respiratory illnesses, and it is one of the leading causes of morbidity and mortality worldwide. An estimated 3 to 5 million people get the flu each year, resulting in 250,000 to 500,000 deaths worldwide (Centres, Cc, July, \& Rt-pcr, 2017). Influenza virus causes seasonal flu annually and pandemic flu sporadically, but there are important differences between the two. Seasonal outbreaks are caused by influenza virus that has undergone a process called antigenic drift, which is an accumulation of mutations over time. Frequent point mutations in the virus surface glycoproteins haemagglutinin (HA) and neuraminidase (NA) results in newly emerging influenza viruses with antigenic variations (Potter, 2001). This has a negative impact on the host immune defense against influenza virus. 
Influenza vaccines must be updated annually to match the circulating antigenic strains and ensure host immunity to influenza. Although seasonal outbreaks of influenza can lead to serious illness, most people in the population have some degree of immunity against the virus due to routine vaccinations against prior antigenic stains. As a result, influenzarelated hospitalizations and deaths due to seasonal outbreaks are usually lower compared to pandemic outbreaks. Pandemic outbreaks are caused by influenza virus that has undergone a process called antigenic shift. It is characterized by major mutations in the influenza virus due to reassortment, which allows two distinct influenza viruses that coinfect the same host to exchange gene segments. Antigenic shift contributes to novel emerging influenza viruses with enhanced virulence responsible for pandemic outbreaks. There have been at least five major influenza pandemics in the past hundred years, namely the Spanish flu (1918-1920), Asian flu (1957-1958), Hong Kong flu (1968-1970), Russian flu (1977-1978) and Swine Flu (2009-2010) (Al Hajjar \& McIntosh, 2010; SaundersHastings \& Krewski, 2016) (Al Hajjar \& McIntosh, 2010; Hinman \& Craven, 1978; Saunders-Hastings \& Krewski, 2016).

In 1918-1919, an influenza A virus (IAV) of H1N1 strain caused the deadliest pandemic outbreaks of the $20^{\text {th }}$ century. It is estimated that approximately 50-100 million people died worldwide (Johnson \& Mueller, 2007; Morens \& Fauci, 2007; Morens, Taubenberger, Harvey, \& Memoli, 2010; Taubenberger \& Morens, 2006). One of the unique features of the 1918 pandemic was the virus's ability to kill young, healthy adults between the ages of 20-40 years (Taubenberger \& Morens, 2006). The second major influenza pandemic of the 
$20^{\text {th }}$ century was the Asian flu that occurred from 1957-1958. The Asian flu was caused by the H2N2 strain of influenza A virus (IAV). It was the least deadly influenza pandemic of the $20^{\text {th }}$ century with an estimated 1-2 million deaths worldwide (Kilbourne, 2006; Saunders-Hastings \& Krewski, 2016). The Hong Kong pandemic, which occurred from 1968-1970, was the last pandemic of the $20^{\text {th }}$ century. The Hong Kong pandemic was estimated to have caused 500,000-2 million deaths worldwide. The influenza A (H3N2) virus was responsible for the Hong Kong pandemic (Cockburn, Delon, \& Ferreira, 1969; Grais, Viboud, Lafont, Miller, \& Simonsen, 2005; Saunders-Hastings \& Krewski, 2016). The Russian flu occurred from 1977-1978 and caused milder symptoms compared to the previous pandemics. The Russian flu was also caused by the H1N1 strain of the influenza A virus. A unique characteristic about the Russian flu was that it mostly affected young people. Additionally, the Russian flu was not caused by a novel strain of influenza virus. It first surfaced in 1950 and reappeared in 1977 (Rozo \& Gronvall, 2015). The Swine flu, which occurred between 2009-2010, was the first influenza pandemic of the 21th century. It was also caused by the influenza A virus (IAV) of H1N1 strain and killed approximately 575,000 people worldwide (Al Hajjar \& McIntosh, 2010; Dotis \& Roilides, 2009; Morens \& Fauci, 2007; Saunders-Hastings \& Krewski, 2016). 
Table 1: List of influenza pandemics from the past one hundred years (Saunders-Hastings \& Krewski, 2016).

\begin{tabular}{|c|c|c|c|}
\hline Pandemic Name & Year of Outbreak & Virus Strain & Number of Death \\
\hline Spanish Flu & $1918-1920$ & H1N1 & 50-100 million \\
\hline Asian Flu & $1957-1958$ & H2N2 & million \\
\hline Hong Kong Flu & $1968-1970$ & H3N2 & \% \\
\hline Swine Flu & $2009-2010$ & H1N1 & \\
\hline
\end{tabular}




\subsection{Types of Influenza Viruses}

There are four different types of influenza virus: influenza A, B, C and D. Influenza A and B are both responsible for annual epidemic and occasional pandemic outbreaks in humans. Influenza A viruses cause more severe disease than other types of influenza. Only influenza A viruses (IAV) can infect diverse hosts such as humans, pigs, horses, minks, seals, birds, wild waterfowl, cattle, cats, tigers, domestic ducks, chickens, turkeys, dogs, bats, quails, and ferrets (Hussain, Galvin, Haw, Nutsford, \& Husain, 2017; Toshihiro Ito \& Kawaoka, 2000; Otte et al., 2007). The influenza A virus (IAV) is classified based on the isoform of the glycoproteins (hemagglutinin (HA) and neuraminidase (NA)) on the surface of the virus. There are 16 different types of hemagglutinins $(\mathrm{H} 1, \mathrm{H} 2, \mathrm{H} 3, \mathrm{H} 5, \mathrm{H} 6, \mathrm{H} 7, \mathrm{H} 8, \mathrm{H} 9$, $\mathrm{H} 11, \mathrm{H} 12, \mathrm{H} 13, \mathrm{H} 14, \mathrm{H} 15$ and 16) and 9 different types of neuraminidases (N1, N2, N3, N4, N5, N6, N7, N8 and N9) (Air, 2006; Gamblin \& Skehel, 2010; Proença-Módena, Macedo, \& Arruda, 2007).

The combination of HA and NA subtypes that commonly cause outbreaks in humans are H1N1, H2N2, and H3N2. These three subtypes (H1N1, H2N2 and H3N2) have all caused pandemic outbreaks in the past. Other subtype combinations of HA and NA have been found to infect various species (dogs, cats, horses, and ferrets). Avian influenza viruses (H5N1, H9N2, H7N7, and H7N9) and swine influenza viruses (H1N1, and H3N2) can infect humans. Influenza B viruses (IBV) were isolated in 1940 (Seleka et al., 2017), they can infect humans, seals, horses, pigs, dogs and ferrets (Bodewes et al., 2013; Borkenhagen et al., 2018; Clara et al., 2013; Sweet, Jakeman, Leone, Russell, \& Tisdale, 2012). Unlike 
influenza A viruses, influenza B viruses cause seasonal outbreaks but not pandemic outbreaks. Additionally, previous studies have revealed that influenza B viruses cause less severe symptoms in humans. Furthermore, influenza B viruses are not classified into subtypes based on the isoforms of the virus glycoproteins (HA and NA). In contrast, influenza B viruses are grouped into two distinct evolutionary lineages: B/Victoria/2/87like viruses and B/Yamagata/16/88-like viruses (Biere, Bauer, \& Schweiger, 2010; Holmes et al., 2013). These two lineages emerged gradually due to antigenic drift in influenza B virus surface glycoprotein hemagglutinin (HA) (Ni, Kondrashkina, \& Wang, 2013). The two lineages co-circulate with influenza A viruses annually. It was reported that influenza B viruses mostly affect children and young adults. Specifically, B/Victoria/2/87-like viruses affect younger populations compared to B/Yamagata/16/88-like viruses (Seleka et al., 2017; van de Sandt et al., 2015).

Influenza C viruses (ICV) were isolated in 1947 (Racaniello \& Palese, 1979). They can infect humans, swine, pigs, and feral dogs (Borkenhagen et al., 2018). Unlike influenza A and $B$ viruses, influenza $C$ virus causes milder symptoms in humans. Influenza $C$ virus primarily affects infants (Itagaki et al., 2006). It has a weak virulence and does not propagate well in cell cultures. Therefore, influenza $\mathrm{C}$ virus is not well studied compared to influenza A and B (Racaniello \& Palese, 1979).

The influenza D virus (IDV) was first isolated in 2011 in the United States (Sheng et al., 2013). However, IDV viruses were also identified in cattle in France and China (Ducatez, Pelletier, \& Meyer, 2015; Jiang et al., 2014). Like influenza C viruses, influenza D viruses 
are not well studied. It has been reported that IDV infect swine, cattle, goats, pigs and sheep (Epperson et al., 2016; Sheng et al., 2013). There are not yet any reports suggesting that influenza D viruses infect humans. Influenza D viruses are more closely related to influenza $\mathrm{C}$ viruses compared to influenza $\mathrm{A}$ and $\mathrm{B}$. Therefore, the first influenza D isolated in pigs in Oklahoma was named C/Oklahoma/1334/2011 (Sheng et al., 2013). More strains of influenza D viruses have been isolated since 2011 (Epperson et al., 2016).

\subsection{Viral Genome}

Influenza $A$

Structurally, influenza A viruses are spherical or filamentous in shape $(100-300 \mathrm{~nm})$ (Bouvier \& Palese, 2008). They belong to the Orthomyxoviridae family of viruses and contain an eight-segmented, negative-sense, single-stranded RNA genome. The genome is organized into eight individual ribonucleoprotein complexes bound to viral nucleoprotein packed into a single viral capsid. In addition, there is an RNA polymerase complex associated with each ribonucleoprotein (Bouvier \& Palese, 2008; Mccauley \& Mahy, 1983). The eight virus segments encode 11 proteins, which are hemagglutinin (HA), neuraminidase (NA), matrix protein $1(\mathrm{M} 1)$, matrix protein $2(\mathrm{M} 2)$, nucleoprotein $(\mathrm{NP})$, non-structural protein 1 (NS1), non-structural protein 2 (NS2; also known as nuclear export protein, NEP), RNA polymerase complex consisting of polymerase acid protein (PA), polymerase basic protein 1 (PB1), polymerase basic protein 2 (PB2), and proapoptotic peptide (PB1-F2). 
The virion is enveloped with a lipid bilayer derived from the host cell plasma membrane. On the outer part of the virion, there are spikes of hemagglutinin (HA) and neuraminidase (NA). HA plays a critical role in virus attachment and virus entry into host cells. HA also mediates the attachment of the virus to sialic acid receptors on the surface of the cells, leading to the release of the viral genome into the host cell. To initiate infection, HA precursor HA0 must be cleaved into HA1 and HA2 by cellular proteolytic enzymes found in the respiratory track. The hydrophobic nature of the $\mathrm{N}$ terminus of the HA2 polypeptide promotes the fusion of the viral endosome with the cell membrane, resulting in the release of the virus into the cytoplasm. NA is an enzyme that cleaves newly synthesized virus from the surface of the host cell. Influenza virus binds to sialic acid receptors on the surface of the cell to enter the host cell. After virus replication, the cleavage of sialic acid receptors by the enzyme, NA, is required for the release of newly formed viral particles from the surface of the host cell.

M1 protein plays an important role in viral budding. M1 has been shown to interact with HA and NA proteins to facilitate viral budding. Furthermore, M1 also provides structural support to the virus membrane by interacting with ribonucleoprotein (RNP) in the virion (Rossman \& Lamb, 2011). M2 is an ion channel that plays a key role in the release of the virus contents into the cytoplasm. M2 has been shown to interact with HA, NA, M1 and NP and may play an important role in viral replication, budding, and assembly. Influenza virus enters cells through a process known as endocytosis. Once inside the cells, M2 lowers the $\mathrm{pH}$ of the viral endosome to facilitate viral uncoating. Additionally, change in the $\mathrm{pH}$ leads to conformational changes in the HA protein, which triggers the fusion of the viral 
endosome with the cell membrane. (Rosmman et al. 2011). The viral nucleoprotein (NP) plays an important role in virus transcription, replication, and packaging. NP is an RNA binding protein that encapsidates the viral genome to form ribonucleoprotein (RNP) molecules. After the virus enters the cell, RNP molecules are released into the cytoplasm, followed by entrance into the nucleus. Once inside the nucleus, RNP molecules initiate virus transcription and replication. NP has been shown to interact with PB1, PB2 and M1 proteins. NP interaction with the viral polymerase complex involves PB1 and PB2, but not PA. (Air, 2006; Rossman \& Lamb, 2011).

The primary role of NS1 protein is to inhibit the host's anti-viral response. Specifically, NS1 limits the production of interferons (IFN) that specialize in blocking the spread of virus inside the cells. Upon virus infection, the innate and adaptive immune systems are activated to eliminate infection. The innate immune system is the first line of defense against infection. It induces type I interferons and other cytokines in the infected cells and non-infected cells to control infection. Type I interferons play a key role in the innate immune response to viral infection. Type I interferons such as interferon- $\alpha$ (IFN- $\alpha$ ), and interferon- $\beta$ (IFN- $\beta$ ) can directly inhibit virus propagation. NS1 protein has been shown to inhibit the production of interferon- $\beta$ (IFN- $\beta$ ), leading to inactivation of key signaling pathways necessary for the induction of anti-viral genes. Hence, NS1 is required for efficient viral propagation (Hale, Randall, Ortin, \& Jackson, 2008).

The primary function of the NS2 protein (also known as NEP) is to mediate the export of the viral ribonucleoproteins (vRNPs) from the nucleus. NS2 interacts with M1 to facilitate 
nuclear export of the vRNPs (O’Neill, Talon, \& Palese, 1998). The influenza polymerase complex comprises PB1, PB2 and PA proteins. PB1, PB2 and PA proteins interact with each other for virus transcription and replication. They are required for viral replication. PB1 plays a central role in virus transcription and replication by facilitating the interaction between the viral RNA (vRNA) and the complementary RNA (cRNA). It also plays an essential role in RNA elongation and contains the amino acid sequence found in other RNA-dependent RNA polymerases. The RNA-dependent RNA polymerase (RdRp) is essential for virus replication (Gonzalez \& Ortin, 1999). PB2 also plays an important role in a process called cap-snatching (Warne et al., 2017). Cap-snatching is a process that involves the cleavage of a nucleotide (10-13 nucleotides) sequence from the 5 ' end of the host mRNA by viral nucleoprotein (vRNA) and using it as a primer for the synthesis of viral transcripts (De Vlugt, Sikora, \& Pelchat, 2018; McCarthy et al., 2009). Additionally, PB2 has been shown to interfere with the innate immune response by inhibiting the host anti-virus response (Long \& Fodor, 2016). The role of PA has not been fully elucidated. However, it has been shown to play an important role in cap-binding, RNA binding, and viral replication (McCarthy et al., 2009). Influenza virus PB-F2 protein is known to play an important role in the innate immune response to infection by inducing cell death (McAuley, Zhang, \& McCullers, 2009; Zamarin, Ortigoza, \& Palese, 2006).

\section{Influenza $B$}

Like influenza A viruses, influenza B viruses belong to the Orthomyxoviridae family. The influenza B virus genome also contains an eight-segmented, negative-sense, single- 
stranded RNA genome. The eight segmented genomes encode 11 proteins which are: HA, NA, NP, M1, NS1, NS2, PB1, PB2, PA, NB and BM2. Influenza A and B have nine proteins (HA, NA, NP, M1, NS1, NS2, PB1, PB2, and PA) in common. BM2 and NB proteins are only specific to the influenza B virus. BM2 protein is an ion channel that is necessary for virus replication (Hatta, Goto, \& Kawaoka, 2004). It has been reported that the NB protein also functions as an ion channel. However, the NB protein is not involved in virus replication (Elderfield et al., 2016; Hatta \& Kawaoka, 2003).

\section{Influenza $C$}

Influenza $\mathrm{C}$ belongs to the Orthomyxoviridae family of viruses. It contains a sevensegmented, negative-sense, single-stranded RNA genome that encodes nine proteins. The proteins are PB1, PB2, P3 (also known as PA), hemagglutinin esterase-fusion (HEF) glycoprotein, NP, M1, CM2, NS1 and NEP. Influenza C virus has 7 proteins (PB1, PB2, PA, M1, NS1, NP and NEP) that are also found in both influenza virus A and B. CM2 and HEF proteins are the only two proteins that are unique to the influenza $\mathrm{C}$ virus. $\mathrm{CM} 2$ protein functions as an ion channel and facilitates uncoating of the virus (Muraki, Okuwa, Himeda, Hongo, \& Ohara, 2013). CM2 is also critical for influenza C virus replication (Takashita et al., 2010). The HEF protein of influenza $\mathrm{C}$ virus has a dual function for glycoproteins (NA and HA) found in influenza A and B (M. Wang \& Veit, 2016). HEF protein has many cellular functions, including receptor binding and receptor destroying activities (Hanika et al., 2005).

\section{Influenza $D$}


Influenza D belongs to the Orthomyxoviridae family of viruses. It also has a sevensegmented, negative-sense, single-stranded RNA genome. Influenza D viruses share approximately 50\% of amino acid sequences with influenza C (Qi et al., 2016). The influenza D genome encodes nine proteins: PB1, PB2, P3, NP, NS, M, influenza D M2 protein (DM2), DM1, and glycoprotein HEF (Asha \& Kumar, 2019a). The influenza D protein, DM2, functions like an ion channel and is essential to virus replication. Additionally, the influenza D virus glycoprotein, HEF, possesses receptor binding and destroying activities (Baioni et al., 2017). 
Figure 1: Cartoon representation of influenza $\mathrm{C}$ and $\mathrm{B} / \mathrm{A}$ virus particles.
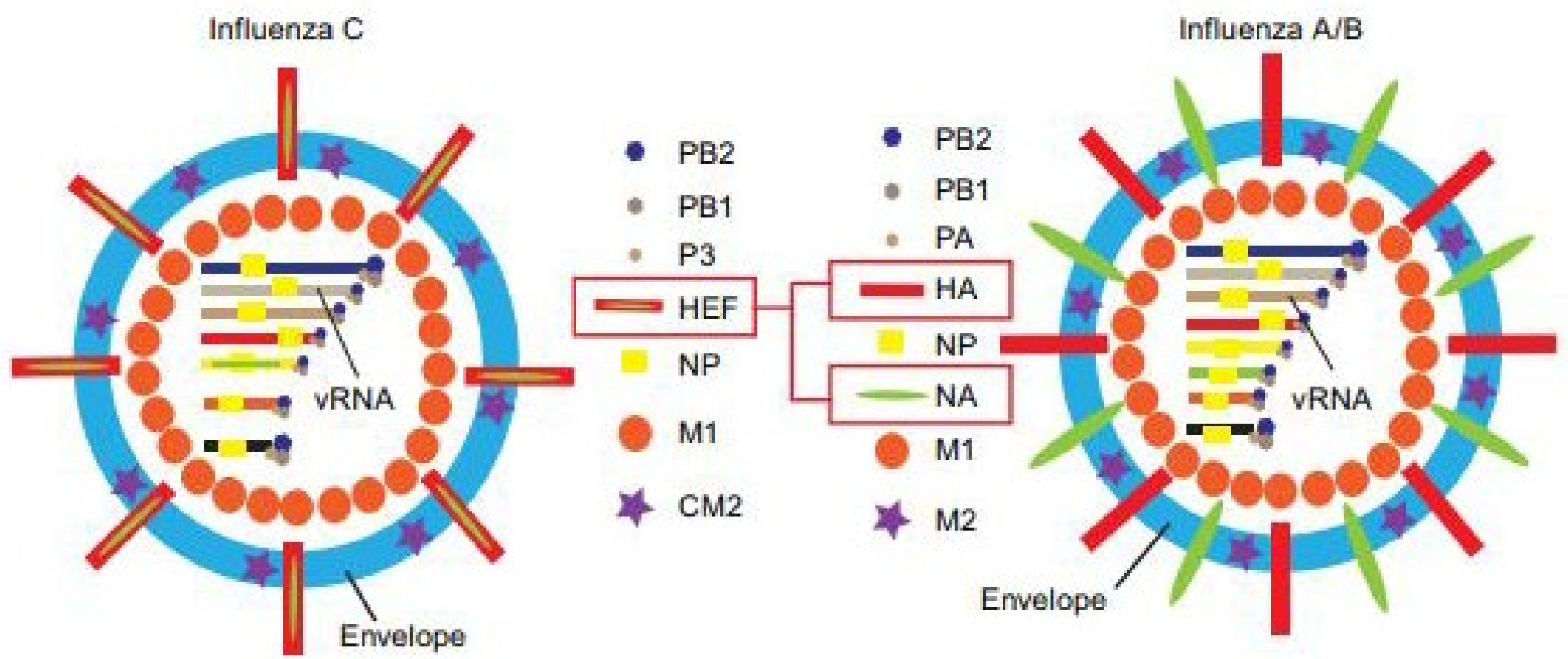

Same symbols are used to depict proteins with the same function. The Hemagglutinin-esterase fusion glycoprotein (HEF) of influenza $\mathrm{C}$ virus has a dual function for glycoproteins neuraminidase (NA) and hemagglutinin (HA) found in influenza A and $B$ viruses. PB1, PB2, PBA, PB1, PB2 and P3 make up the polymerase proteins of influenza A/B and influenza C respectively, that form a complex with nucleoprotein (NP) and the viral RNA-segment (vRNA). M1 represents the matrix protein and M2 is the proton channel of influenza A/B. M1 represents the matrix protein and CM2 is the proton channel of influenza C (M. Wang \& Veit, 2016). 
Figure 2: Cartoon representation of influenza D virus particles.

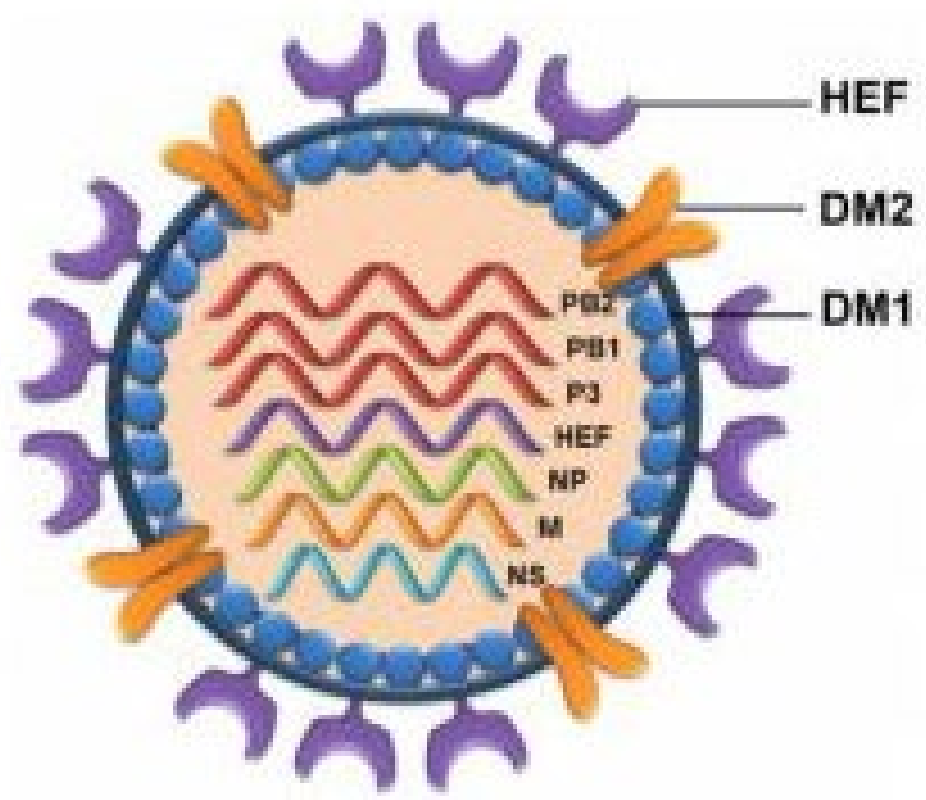

Influenza D virus particle is an envelope virus containing 7 RNA segments. The virion size ranges from 80 - $120 \mathrm{~nm}$ in diameter. The Hemagglutinin-esterase fusion glycoprotein (HEF) of influenza D virus has a dual function for glycoproteins neuraminidase (NA) and hemagglutinin (HA) found in influenza A/ B viruses. HEF mediates virus attachment and entry into the host cells. DM2 protein is a protein channel (Asha \& Kumar, 2019b). 


\subsection{Influenza Virus Receptors}

Influenza virus must bind to receptors on the surface of the host cell to gain entry. Nacetylneuraminic acid (also known as sialic acid receptors) are well-known receptors of influenza virus. Influenza viruses bind to the sialic acid containing molecules, namely $\alpha 2,3$ and a2,6-linked sialic acids (García-Sastre, 2010). Sialic acids are cell surface monosaccharides found on the terminal end of asparagine-linked-glycans (N-glycans), and oxygen-linked-glycans (O-glycans). They are found on the cell surface glycoproteins, glycolipids, and glycosphingolipids (Varki \& Varki, 2007). The efficiency of influenza virus infection in humans and animals is determined by the specificity and the quantity of sialic acid receptors present. Numerous studies show that in humans, influenza viruses such as $\mathrm{H} 1 \mathrm{~N} 1$, and $\mathrm{H} 3 \mathrm{~N} 2$ preferentially bind to $\alpha 2,6$-linked sialic acids, whereas avian influenza viruses, including H5N1, preferentially bind to $\alpha 2,3$-linked sialic acids (Kimble, Nieto, \& Perez, 2010). Studies showed that both $\alpha 2,3$ and $\alpha 2,6$-linked sialic acids are expressed in the human respiratory tract. However, the distribution of $\alpha 2,3$ and $\alpha 2,6-$ linked sialic acids varies throughout the human respiratory tract. Previous studies reported that $\alpha 2,6$-linked sialic acids are predominantly expressed in the upper respiratory tract and $\alpha 2,3$-linked sialic acids are predominantly expressed in the lower respiratory track. Specifically, it has been reported that $\alpha 2,6$-linked sialic acids are expressed in non-ciliated epithelial cells, whereas $\alpha 2,3$-linked are expressed in ciliated epithelial cells. Numerous reports suggest that the low expression of $\alpha 2,3$-linked sialic acids in the human upper respiratory tract prevents human susceptibility to avian influenza viruses. However, recent studies have demonstrated that both $\alpha 2,3$ and $\alpha 2,6$-linked sialic acids can be found in non-ciliated epithelial cells as well 
as ciliated epithelial cells. Nicholls and colleagues found that both $\alpha 2,3$ and $\alpha 2,6$-linked sialic acids were present in ciliated epithelial, metaplastic epithelial cells, goblet cells, and alveolar cells. Most importantly $\alpha 2,3$-linked sialic acids were highly expressed in neonatal pneumocytes and neonatal bronchus. Overall, high levels of $\alpha 2,3$ sialic acids and low levels of $\alpha 2,6$-linked sialic acids were found in the respiratory tract of young children. In comparison, high levels of $\alpha 2,6$-linked and low levels of $\alpha 2,3$-linked sialic acids were found in the adults respiratory tracts (Nicholls, Bourne, Chen, Guan, \& Peiris, 2007). High levels of $\alpha 2,3$-linked sialic acids in the respiratory tract could lead to susceptibility to highly pathogenic avian influenza virus infection in children.

There are numerous reports about avian influenza virus infections in humans. Infections with highly pathogenic avian influenza (HPAI) virus (H5N1 and H7N7) and low pathogenic avian influenza (LPAI) H9N2 have been reported in humans (Auewarakul et al., 2007; De Graaf \& Fouchier, 2014; Peiris, De Jong, \& Guan, 2007). H5N1 influenza virus infection has been reported to cause respiratory failure and a high mortality rate in children and young adults (Oner et al., 2012). H7N7 infection in humans has been reported in individuals working with poultry (Bos et al., 2010). H9N2 influenza virus infections have also been reported in children (Butt et al., 2005). Numerous studies suggest that avian influenza virus infections in humans often results from poultry-to-human transmission, and pig-to-human transmission (Butt et al., 2005; Ma, Kahn, \& Richt, 2009). There are also increasing reports suggesting that avian influenza viruses can bind to receptors found in humans. Auewarakul and colleagues showed that H5N1 influenza virus has a strong binding affinity to the $\alpha 2,6-$ linked sialic acid receptor in humans (Auewarakul et al., 2007). 
Furthermore, Stevens and colleagues demonstrated the binding of avian and swine influenza virus hemagglutinins (H5 and H9) to human sialic acid receptor analogues (Ha, Stevens, Skehel, \& Wiley, 2002). The transmission and replication of influenza depends on the prevalence of the sialic acid receptors. Influenza virus with high affinity to both $\alpha 2,3$ and $\alpha 2,6$-linked sialic acid receptors could have a devastating impact in humans.

$\alpha 2,3$-linked and $\alpha 2,6$-linked sialic acids are also co-expressed in many other species including waterfowl, ducks, pigs, turkeys, geese, pheasants and quails. They serve as a reservoir for influenza virus replication. In ducks, $\alpha 2,3-$ linked sialic acids are found in the trachea, lung, and large intestine; whereas, $\alpha 2,6$-linked sialic acids are found in the lung. In pheasants, $\alpha 2,3$ and $\alpha 2,6$-linked sialic acids are found in the trachea, lung, and large intestine (Kimble et al., 2010). High levels of $\alpha 2,3$-linked and $\alpha 2,6$-linked sialic acids provide a unique environment for influenza virus reassortment and replication. For example, pigs are known as "mixing vessels" because human influenza virus and avian influenza virus can co-infect pigs. Co-infection of human and avian influenza viruses in pigs can result in the reassortment of influenza gene segments and generation of a novel strain of influenza virus (Ma et al., 2009). 
Figure 3: Types of sialic acids and glycosidic bonds.

\section{Neu5Ac}

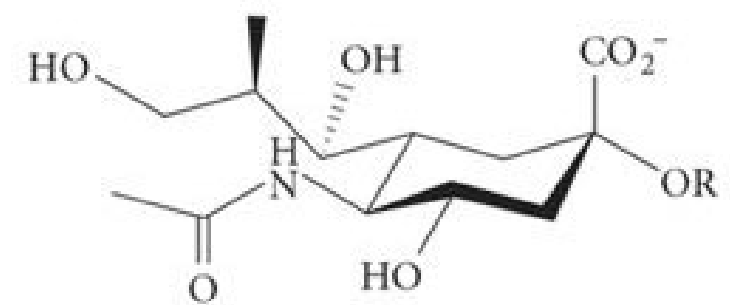

\section{Neu5Gc}

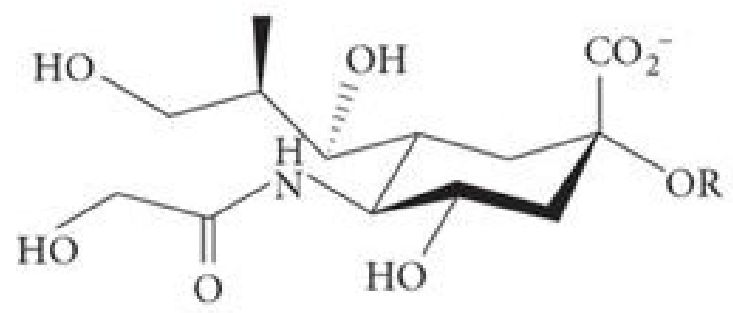

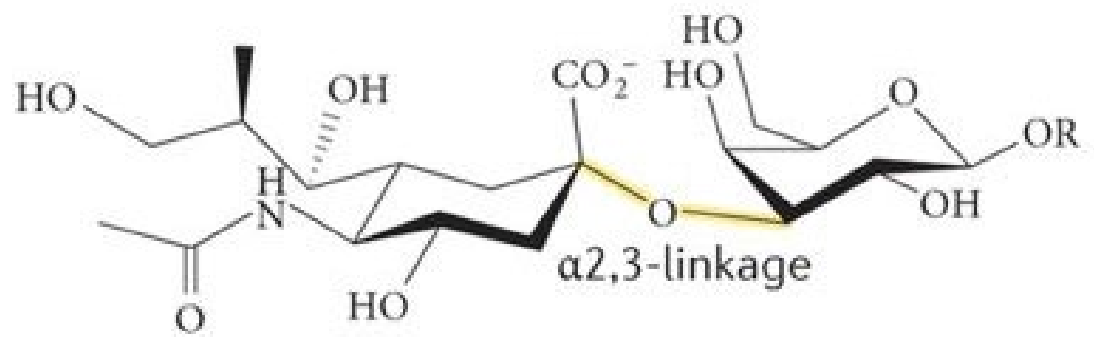

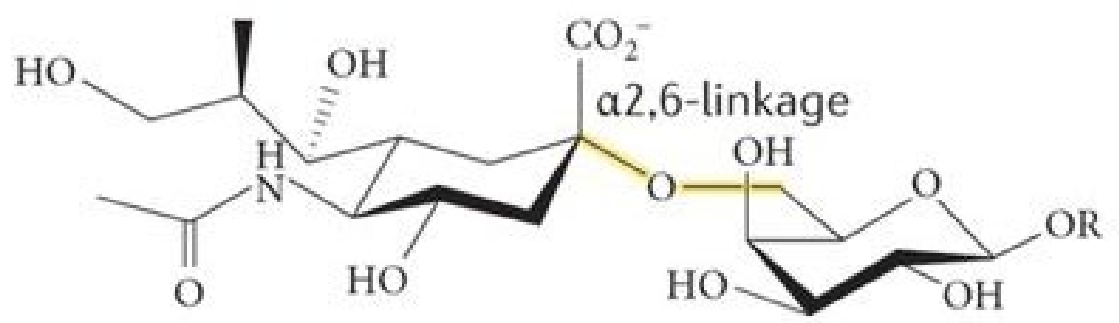

Sialic acids are 9 carbon monosaccharides derived from neuraminic acid. $\alpha 2,3$-linked and $\alpha 2,6$-linked to galatose residue or to Nacetylgalactosamine are the most common types of sialic acid linkages (Stencel-Baerenwald, Reiss, Reiter, Stehle, \& Dermody, 2014). 


\subsection{Influenza Life Cycle}

Influenza virus replication involves multiple stages, including viral attachment and entry, release of viral genome into the host cell, viral transcription and replication, viral protein synthesis, virus assembly, and the release of new viral progenies. New infectious viral progenies must contain all the necessary genomic materials required to infect a new cell.

\section{Virus attachment and entry}

In order to initiate infection, influenza virus must first attach to the cell surface via $\alpha 2,3-$ linked and $\alpha 2,6$-linked sialic acid receptors. The attachment of the virus to the cell surface receptor is mediated by influenza virus glycoprotein hemagglutinin. HA binds to sialic acids and enters cells through receptor-mediated endocytosis. HA is a homotrimeric glycoprotein found on the surface of the virus. To initiate infection, host proteases cleave HA precursor, HA0, into two distinct subunits: HA1 and HA2. The HA1 contains the receptor binding domain and HA2 contains the fusion peptide. Furthermore, HA1 binds to the sialic acid receptors on the host cells and HA2 mediates membrane fusion (Samji, 2008).

\section{Endocytosis and fusion}

Once inside a cell, the $\mathrm{pH}$ of the endosome changes from neutral in the early endosome to acidic in the late endosome due to the activation of a M2 protein ion channel. M2 ion channels mediate the acidification of the late endosome through the influx of protons and efflux of $\mathrm{K}^{+}$from the virus core. The acidification of the viral core weakens the M1-vRNP. 
Low $\mathrm{pH}$ inside the endosome induces the fusion of the viral endosomal membrane with the host cell membrane, leading to the release of vRNPs into the cell. vRNPs are then transported into the nucleus for virus transcription and replication (Manzoor, Igarashi, \& Takada, 2017).

\section{Viral replication and transcription}

Influenza viruses contain a negative-sense, single-stranded vRNA-genome. The negativesense vRNA genome must be transcribed into a positive-sense vRNA genome for virus replication and transcription to occur. During virus replication, the negative-sense vRNA genome serves as a template to generate complementary RNAs (cRNAs) and virus mRNAs using RNA dependent RNA polymerase ( $R d R p)$. Thus, $R d R p$ is responsible for transcription and replication of vRNA. The viral mRNAs are then translated into viral proteins and new viral RNAs (vRNAs) are synthesized from the cRNAs (Dou, Revol, Östbye, Wang, \& Daniels, 2018; Scull \& Rice, 2010).

\section{Viral protein synthesis}

Influenza virus requires host cell machinery for viral protein synthesis. The synthesis of viral mRNA occurs in the nucleus, followed by mRNA translation in the cytoplasm (Samji, 2008).

\section{Viral assembly and budding}

Viral assembly and budding are the later stages of the influenza virus life cycle. The exact mechanism of virus budding is not well understood. Studies show that influenza viral 
assembly and budding occur in the apical side of virus-infected, polarized epithelial cells. The process of budding begins by the transportation of all viral components to the apical site of epithelial cells. Infected cells contain a lipid raft domain that facilitates virus budding (Rossman \& Lamb, 2011). It has been shown that influenza virus matrix protein 1 (M1) plays a major role in viral assembly and budding. M1 induces the process of virus budding and formation of virus like particles (VLPs) through the interaction between the viral nucleocapsid and viral glycoproteins (Ali, Avalos, Ponimaskin, \& Nayak, 2002; Gomez-Puertas, Albo, Perez-Pastrana, Vivo, \& Portela, 2002). 
Figure 4: Cartoon representative of influenza A virus structure and influenza virus life cycle.

A.

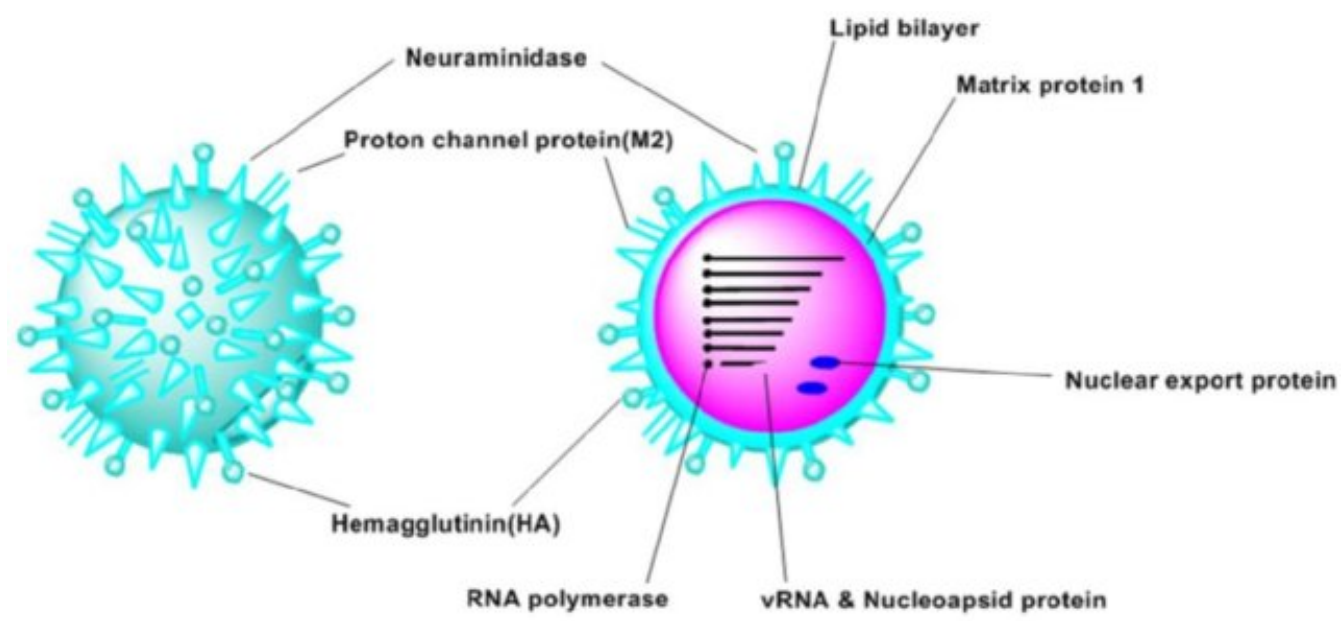

B.

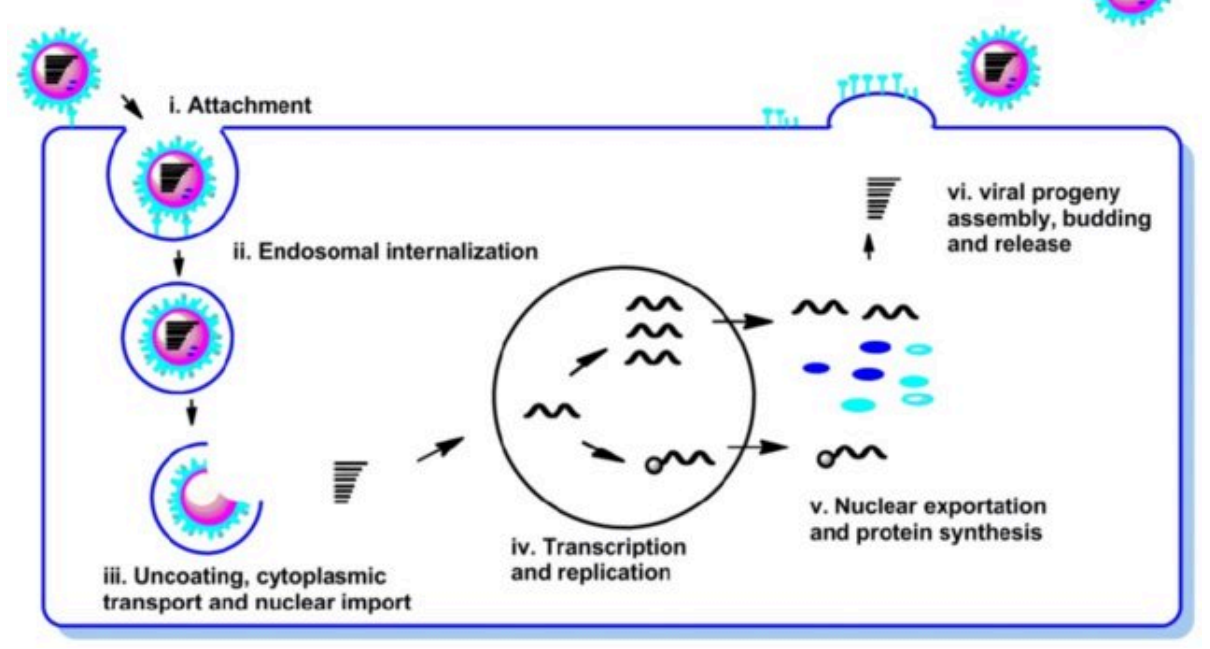


A. Influenza A virus particle is an envelope virus containing proteins encoded by eight segments of negative-strand RNA. Influenza virus proteins include hemagglutinin (HA), neuraminidase (NA), nucleoprotein (NP), RNA polymerase (PA, PB1, PB2), matrix protein 1 (M1), proton channel M2, nonstructural protein 1 (NS1) and nuclear export protein (NEP, NS2). B. Cartoon representation of the influenza virus life cycle showing early and late stages of the cycle including, virus attachment, endosomal internalization, virus encoding followed by cytoplasmic transport and nuclear transport, transcription and replication, protein synthesis, and viral assembly and budding (X. Wu et al., 2017). 


\subsection{Clinical features and pathogenesis of influenza}

Influenza virus predominately replicates in the lower and upper respiratory tract. However, other cell types such as human natural killer cells and dendritic cells, can also be infected by influenza virus (Mao et al., 2009; Smed-Sörensen et al., 2012). In humans, influenza virus is transmitted in three ways, including: direct transmission, inhalation of virus-laden aerosols, and indirect transmission. Clinical symptoms of influenza virus infection include coughing, fever, headache, sore throat, nasal congestion, shortness of breath, and weakness. These symptoms can occur within 24 hours to 5 days after infection (Caini, Kroneman, Wiegers, El Guerche-Séblain, \& Paget, 2018). Influenza virus infection can result in serious complications such as pneumonia, pulmonary edema, acute respiratory distress syndrome (ARDS), respiratory failure and death (Brauer, Chen, Brauer, \& Chen, 2016). 


\subsection{Risk Factors}

Age

There are several risk factors associated with influenza virus infection. One of the risk factors is age. Influenza viruses cause significant illness in infants, young children and the elderly. Nair and colleagues used meta-analysis to estimate the global burden of influenza virus infections in children. It has been reported that an estimated 28,000 to 111,500 children under the age of 5 years old died from influenza-associated acute lower respiratory infections (ALRI) in 2008. Furthermore, it was estimated that $99 \%$ of these deaths occurred in developing countries (Nair et al., 2011). Newborn infants have also been shown to be at a higher risk of influenza virus infection. In the United States, it was estimated that approximately 7.2 out of 1000 infants under six months old are hospitalized each year due to influenza-associated complications (MacDonald \& Bortolussi, 2009). Influenza viruses are also associated with a high mortality rate in elderly people. Studies show that 17 out of 100,000 elderly people died from influenza-related diseases each year in the United States. Additionally, $87.9 \%$ of these deaths were due to pneumonia and influenza related causes (Frieden et al., 2010).

\section{Pregnancy}

Numerous studies have shown that pregnant women are at an increased risk for getting an influenza virus infection. An analysis of hospital admission records of pregnant women from 1994 to 2000 revealed that an estimated 150 pregnant women per 100,000 are hospitalized annually due to influenza virus infections (Schanzer, Langley, \& Tam, 2007). Increased severity of influenza virus infections was also observed in a two population- 
based cohort study of pregnant women in Australia, New Zealand, and the United Kingdom (UK) from 2009 to 2010. However, increased admission to an intensive care unit (ICU) due to an influenza virus infection, was more prevalent in pregnant women in Australia and New Zealand than in the UK (Knight et al., 2011). It has been reported that influenza virus infection in pregnancy is associated with adverse neonatal outcomes such as low birth rate, premature birth, and infant death (T. J. Doyle, Goodin, \& Hamilton, 2013). The 2009 pandemic influenza A virus has been reported to be associated with high morbidity and mortality in pregnant women. In a case study conducted by the Centers for Disease Control and Prevention (CDC), it was found that six pregnant women out of 34 confirmed influenza cases died from influenza-associated complications (Jamieson et al., 2009).

\section{Immunosuppression}

It has also been reported that immunosuppressed patients are at a high risk for severe influenza virus infections. Increased death from pneumonia has been reported in immunosuppressed patients infected with influenza virus (Schnell et al., 2010).

\section{Environmental factors}

Studies have shown that environmental factors such as temperature, humidity, and air pollutants affect the transmission and spread of influenza virus (Pica \& Bouvier, 2012). Numerous studies have revealed that influenza virus infections are linked to environmental pollutants such as cadmium, ozone, diesel exhaust, and arsenic. Checconi and colleagues showed that exposure to heavy metal cadmium enhances influenza virus infection in 
MDCK cells (Checconi et al., 2013). Exposure to ozone and diesel exhaust have also been found to enhance influenza virus infections in human respiratory epithelial cells (Jaspers et al., 2005; Kesic, Meyer, Bauer, \& Jaspers, 2012). Epidemiological and experimental studies show that exposure to environmental levels of arsenic in drinking water is associated with severe outcomes from influenza virus infections (Farzan et al., 2016; Kozul, Ely, Enelow, \& Hamilton, 2009).

\subsection{Prevention and Treatment}

\section{Prevention}

Numerous studies suggest that vaccination is the best method for the prevention of influenza virus infection. There are different types of influenza vaccines including live attenuated influenza vaccines (LAIV), inactivated influenza vaccines (IIV), and recombinant influenza vaccines (RIV) (J. R. Chung et al., 2016; Weir \& Gruber, 2016). In the United States, vaccines are regulated by the Food and Drug Administration (FDA). Specifically, the Center for Biologics Evaluation and Research (CBER), a branch of the FDA, is responsible for ensuring the safety and efficacy of vaccines (Marshall \& Baylor, 2011). In the United States, inactivated influenza vaccines have been available since 1942. In 2003 and 2013, the FDA approved live attenuated influenza and recombinant influenza vaccines, respectively (Barberis et al., 2016).

\section{Treatment}

Several FDA-approved anti-influenza drugs are in the market for the treatment of influenza virus infections. The first major class of anti-influenza drugs are the neuraminidase 
inhibitors (NAIs) such as zanamivir and oseltamivir. Zanamivir and oseltamivir are clinically used for prevention and treatment of influenza viruses. Neuraminidase inhibitors inhibit the neuraminidase enzyme and prevent the release of newly formed virions (Mckimm-Breschkin, 2013). The second major class of anti-influenza drugs are the adamantanes. The adamantanes (amantadine and rimantadine) block the influenza virus protein M2 ion channel and inhibit the release of vRNP, which prevents virus replication and transcription (Stiver, 2003).

\subsection{Arsenic}

Arsenic is a naturally occurring element commonly found in minerals. For centuries, it has been more notoriously known for its use as a poison to commit murder. The most famous arsenic-related death is the poisoning of the French Emperor, Napoleon Bonaparte, in the $19^{\text {th }}$ century. Arsenic was also reported to be used to poison the Chinese Emperor,

Guangxu, in the $20^{\text {th }}$ century, causing his death (D. Doyle, 2009). In fact, arsenic was notoriously known as "king of poisons" and "poison of kings" because of its use in committing murder (Hughes, Beck, Chen, Lewis, \& Thomas, 2011).

In the periodical table arsenic (As) is number 33 and has an atomic mass of 74.92. Arsenic is a metalloid that naturally occurs in the Earth's crust and is widely distributed in nature (Reimann, Matschullat, Birke, \& Salminen, 2009). Arsenic is considered a metalloid because it has properties that are a mixture between metal and non-metal. It can be found under group 5A. Arsenic can exist in four oxidation states such as $-3,+3,0$, and +5 . Elemental arsenic can be combined with other elements on the periodical table to form 
various arsenicals. Inorganic arsenic and organic arsenic are the two main forms of arsenic compounds. Inorganic compounds contain arsenic and other elements such as oxygen. Organic arsenic compounds contain arsenic and other elements such as carbon. Both the organic and inorganic compounds have negative health outcomes in humans. However, inorganic arsenic compounds are more toxic than organic arsenic compounds.

The predominate forms of inorganic arsenic are trivalent arsenic $\left(\mathrm{As}^{3+}\right.$ and pentavalent arsenic $\left(\mathrm{As}^{5+}\right)$. Trivalent arsenicals are known to be more potent and toxic than pentavalent arsenicals. Trivalent arsenicals include arsenite, arsenic trioxide, monomethylarsonous acid and dimethylarsenous acid. The exact mechanism of trivalent arsenical toxicity is still unknown, but many mechanisms have been proposed. Trivalent arsenicals react with sulfur-containing proteins to inhibit their functions and/or activities, leading to toxicity. Arsenic induced toxicity has also been linked to the generation of reactive oxygen species (ROS) (Hughes et al., 2011; Kulshrestha, 2014). Pentavalent arsenicals include arsenate, arsenic pentoxide, monomethylarsenic acid, trimethylarsine oxide, arsenilic acid, and arsenobetaine. Arsenic biotransformation plays an important role in arsenic elimination and detoxification. Pentavalent arsenicals are less potent and are reduced to trivalent arsenic prior to elimination (J. Ghosh \& Sil, 2015).

In the environment, arsenic exists as a constituent of 245 minerals such as lead, zinc, copper, gold and nickel in sulfide ore minerals (Shen, Li, Cullen, Weinfeld, \& Le, 2013). There are several sources that contribute to the release of high concentrations of arsenic in the environment. Besides natural sources, arsenic can be released in the environment 
through anthropogenic sources, mining activities, industrial activities, metal processing, fertilizer, pesticides, volcanic activities, geothermal processes, and herbicides (J. Y. Chung, Yu, \& Hong, 2014).

\subsection{Sources of Arsenic Exposure}

Arsenic in Groundwater

Arsenic can be found in sedimentary rocks, soil, water, plants, food, air and all living organisms. Humans are exposed to arsenic through drinking water, food, and air. Millions of people are exposed to arsenic through drinking water, making it the major source of arsenic poisoning worldwide. It has been estimated that $130-200$ million people in 70 countries are exposed to a high level of arsenic through drinking water beyond the safe Maximum Contaminant Level (MCL). The World Health Organization (WHO) and the United States Environmental Protection Agency (EPA) standard for safe levels of arsenic in drinking water is 10 parts per billion (10 ppb) (Minatel et al., 2018; Van Halem, Bakker, Amy, \& Van Dijk, 2009). High levels of arsenic in groundwater have been found in Bangladesh, China, India, Europe, South America, Central America and in the United States (C. M. Steinmaus et al., 2013).

The public health impact of arsenic exposure in Bangladesh has been the most devastating in history. Approximately 80 million people are exposed to unsafe levels of arsenic in Bangladesh. For many years, people from Bangladesh relied on surface water as a source of drinking water. Consequently, surface water was contaminated with microorganisms 
known to cause water-borne diseases in humans. Among those diseases are hepatitis A and E, typhoid fever, cholerae, and malaria. To reduce pathogen related diseases and mortalities associated with drinking contaminated surface water, tube wells were installed in rural communities in Bangladesh beginning in the 1970s. This effort of installing millions of tube-wells in rural communities in Bangladesh went from the 1970s to the 1990s. The United Nations of Children's Fund's (UNICEF) collaborated with Bangladesh's Department of Public Health and Engineering (DPHE) to undertake this project and bring clean, unpolluted water to the Bangladeshi people. It has been estimated that approximately 8.6 million tube wells were installed from the 1970 s to the 1990 s. The tube-wells were never tested for arsenic contamination at the time of installation. In 1992, arsenic in drinking water was discovered by the School of Environmental Studies (SOES) after millions of Bangladeshi people had been poisoned for nearly 20 years. Recently, it was found that half of these tube-wells were tested for arsenic contamination and 1.4 million tube-wells contained unsafe levels of arsenic ( $>50 \mathrm{ppb})$. However, a Bangladeshi government agency known as Bangladesh Atomic Energy Commission, has reported unsafe arsenic levels up to 150 - 200 ppb in tube-wells (Chakraborti et al., 2015; Flanagan, Johnston, \& Zheng, 2012; Allan H Smith, Lingas, \& Rahman, 2000; Uddin \& Huda, 2011).

Currently, more than 5 million people in India are exposed to arsenic at a concentration (50ppb) higher than the WHO recommendation. West Bengal has been reported to be the area most affected by arsenic poisoning in India, but many districts in India are also affected (A. Kumar et al., 2016). For example, there are over 1 million tube-wells in the south-western districts of Punjab that contain unsafe levels of arsenic. The arsenic 
concentration in these were reported to be about 20 - to 30 -fold higher than the WHO recommended levels of 10 ppb (Hundal, Singh, \& Singh, 2009; Shrivastava, 2016). It has been estimated that approximately 19.6 million people in China could be exposed to unsafe levels of arsenic through drinking water (Berg et al., 2013). In the 1960s, many deep wells were installed in China to encourage people to stop getting drinking water from contaminated surface water. However, many of these wells contained unsafe levels (50 ppb) of arsenic beyond the WHO levels of $10 \mathrm{ppb}$. For example, based on a study conducted on arsenic exposure in 16 provinces, 400,000 wells were found to be contaminated with high levels (50ppb) of arsenic, affecting nearly 600,000 people. Further analysis of multiple provinces revealed that 3.34 million people were exposed to arsenic at very high concentrations of $50 \mathrm{ppb}$ to $100 \mathrm{ppb}$ and 2.29 million people were exposed to a concentration that exceeded $100 \mathrm{ppb}$ (Yu, Sun, \& Zheng, 2007).

In northern Chile, more than 250,000 people were exposed to unsafe levels of arsenic in drinking water from 1958 to 1970 (C. M. Steinmaus et al., 2013). People in northern Chile were exposed to very high levels of arsenic in the beginning of the 1960 s when river water from the Andes Mountains became the primary source of drinking water in the city's water supply. Water from the Andes Mountains was found to contain a high concentration of arsenic $(860 \mathrm{ppb})$, resulting in 13 years of arsenic poisoning in the large population of people. Arsenic-removal plants were installed in the 1970s to decrease the level of arsenic contamination in the city water supply (C. \& A.M., 2006; C. M. Steinmaus et al., 2013). 
Arsenic contamination has also been a problem in Europe. Elevated arsenic levels were reported in many European countries such as Greece, Serbia, Hungary, Italy, and Ireland (Van Halem et al., 2009). Italy is one of the European countries most affected by arsenic poisoning in the drinking water supply. In 128 Italian municipalities, a high arsenic concentration of 20 to $50 \mathrm{ppb}$ was found in drinking water (Davoli et al., 2015). Currently, the European Union (EU) and the WHO recommend maximum arsenic levels in drinking water to be $10 \mathrm{ppb}$ (Duarte, Cardoso, \& Alçada, 2009).

Many people who live in the rural parts of the United States get their drinking water from private wells. It has been reported that these private wells contained unsafe levels of arsenic at a concentration greater than $10 \mathrm{ppb}$. According to the WHO and the EPA, the maximum contaminant levels of arsenic in drinking water is $10 \mathrm{ppb}$ in the U.S. (Ayotte, Medalie, Qi, Backer, \& Nolan, 2017). The U.S. Geological Survey (USGS) estimated that approximately 44.1 million in the U.S. use private wells. Among these private well users, 2.1 million people are exposed to arsenic at a concentration greater that $10 \mathrm{ppb}$. Populations of people from 49 states such as Arizona, California, Idaho, New Mexico and New Hampshire (Ayotte et al., 2017) were reported to be exposed to high levels of arsenic (>10 $\mathrm{ppb})$.

\section{Arsenic in Food}

Arsenic poisoning through drinking water has been a public health concern for many years. Therefore, a lot has been done by governmental agencies to implement policies in order to lower the amount of arsenic in drinking water. A second main source of arsenic in humans 
is through food. Inorganic arsenic can be found in foods such as rice, rice products, fruits, vegetables, fruit juices, baby foods and formulas. Organic arsenic can be found in seafood such as fish and it is less toxic than inorganic arsenic. Rice has been shown to contain more inorganic arsenic from the soil compared to other grains. The EPA-recommendation for the maximum arsenic contaminant levels in food is $5 \mathrm{ppb}$. The Food and Drug Administration (FDA) regulates the amount of toxic chemicals in foods such as lead, cadmium, mercury and arsenic. The EPA recommended dose of arsenic in rice is 0.3 $\mu \mathrm{g} / \mathrm{kg} /$ day (Marcason, 2015). Millions of people living in Asia are known to have high rice consumption. However, high concentrations of arsenic have been found in rice in places where arsenic poisoning is a major public health concern in Asia. For example, unsafe levels of arsenic at a concentration of $0.561 \mathrm{mg} / \mathrm{kg}$ to $1.072 \mathrm{mg} / \mathrm{kg}$ were found in different types of rice in Bangladesh (Aziz, Ullah, \& Ullah, 2015). There is no regulation of arsenic in food in Bangladesh. However, these levels exceeded the EPA and the China food standard limit of arsenic which is $0.15 \mathrm{mg} / \mathrm{kg}$ (Aziz et al., 2015).

Apple juice is known to be a source of inorganic arsenic contamination in food. The Food and Drug Administration (FDA) monitors the levels of arsenic in apple juice. The FDA recommended dose of arsenic in apple juice is $10 \mathrm{ppb}$. The source of arsenic in apple juice comes from high levels of arsenic in soil, water and the use of arsenic containing pesticides on land (Food and Drug Administration (FDA), 2013). A recent study was conducted to quantify arsenic concentration in grape products such as apple juice and wine. The average concentration of arsenic in apple juice samples were between $3.06 \mathrm{ppb}$ to $22.5 \mathrm{ppb}$. 
Furthermore, high concentrations of arsenic were found in wine at a concentration between $17 \mathrm{ppb}$ to $37 \mathrm{ppb}$ (NARUKAWA, IWAI, \& CHIBA, 2018).

Arsenic in the Air

Inorganic arsenic is the most predominant form of arsenic in the air. However, organic arsenic is also present. Under the Clean Air Act, the EPA classified arsenic as an air pollutant. It has been reported that humans are exposed to arsenic at a concentration between 40 to $90 \mathrm{ng}$ per day in polluted areas (J. Y. Chung et al., 2014). Arsenic is released into the air from natural sources, anthropogenic sources, and industrial activities.

\section{Arsenic as Medicine}

It has been reported that arsenic has been used for therapeutic purposes for more than 2,400 to 3000 years (Antman, 2004; D. Doyle, 2009). Arsenic was used by Hippocrates (460-377 BC) to treat various diseases. Hippocrates is wildly recognized as the father of modern medicine (Yapijakis, 2009). In 1878, Thomas Fowler discovered potassium bicarbonatebased arsenic trioxide $\left(\mathrm{As}_{2} \mathrm{O}_{3}\right)$ that was used to treat various diseases (D. Doyle, 2009). In 1910, Paul Ehrlich discovered Salvarsan, an arsenic-based compound, that was effective in the treatment of syphilis and trypanosomiasis. Salvarsan was used to treat these diseases until the discovery of penicillin in the 1940s by Alexander Fleming (Tan \& Tatsumura, 2015). The arsenic-based drug called Trisenox (Cell Therapeutics, Inc., Seattle, WA) was approved by the United States Food and Drug Administration (FDA) to treat acute promyelocytic leukemia (APL) (S. Kumar, Brown, \& Tchounwou, 2018; Shooshtary, Behtash, \& Nafisi, 2015). 


\subsection{Health Effects of Arsenic Exposure}

\section{Acute Exposure}

Inorganic arsenic is extremely toxic, and symptoms can occur rapidly after ingestion. Acute arsenic toxicity can occur through inhalation and/or ingestion of arsenic. Once inside the body, arsenic is observed in the gastrointestinal tract and is rapidly distributed to major organs such as the lungs, kidneys, bladder, skin and the liver (Lai, 2005). Acute arsenic toxicity is associated with many symptoms such as vomiting, abdominal pain, diarrhea and death. Acute arsenic poisoning can lead to multiorgan failure and death within 14 hours after ingestion (Tournel et al., 2011).

\section{Chronic Exposure}

Chronic exposure to arsenic has been linked to many diseases such as cancer, skin lesions, developmental effects, cardiovascular disease, neurotoxicity, and diabetes (Chen et al., 2011; Allan H. Smith et al., 2018; Tyler \& Allan, 2014). Arsenic and arsenic-based compounds have been classified as a Group 1 carcinogen to humans by the International Agency for Research on Cancer (IARC) (Martinez, Vucic, Becker-Santos, Gil, \& Lam, 2011). Arsenic has been shown to target diverse organs such as the heart, lungs, liver, kidneys, skin, bladder, and the lymph nodes. Chronic arsenic poisoning has caused devastating health outcomes worldwide. The population of Bangladesh has experienced the worst arsenic poisoning. The primary symptoms of arsenic poisoning are skin lesions caused by chronic exposure to high concentrations of arsenic. These skin lesions can begin to appear 10 years after exposure. In Bangladesh, where people are exposed to high levels 
of arsenic, many people have developed skin conditions such as keratosis, hyperpigmentation, and hypopigmentation. Palmar and solar keratosis are important diagnostic criterion of arsenic poisoning (Milton, Hasan, Rahman, \& Rahman, 2001). Consequently, chronic arsenic exposure has been associated with skin cancer. Arsenic exposure has been linked to Bowen's disease, actinic keratosis, invasive basal carcinoma and squamous cell carcinoma (Wollina, 2016).

Arsenic exposure has also been associated with other cancers such as lung, bladder, kidney, prostate and cervical cancer. Smith et al. showed that lung, bladder and kidney cancer mortality in men and women remained high 40 years after exposure to an unsafe concentration of arsenic in the region of northern Chile (Allan H. Smith et al., 2018). Increased mortality rates due to bladder cancer have been observed in human populations exposed to high concentrations of arsenic through drinking water. Epidemiological studies in countries such as Bangladesh, Chile, and India revealed a direct link between arsenic exposure and bladder cancer. A population based-cohort study in regions of the U.S (New Hampshire, Maine and Vermont) revealed that a 20\% increased rate of bladder cancer incidents was associated with unsafe levels of arsenic in drinking water from private wells (Baris et al., 2016). Inorganic arsenic exposure has also been shown to be a risk factor for an increased rate of kidney cancer. For example, Yuan et al. investigated kidney cancer risk in arsenic-exposed and unexposed human populations in northern Chile. The Region II of northern Chile was exposed to unsafe levels of arsenic ( $90 \mathrm{ppb}-870 \mathrm{ppb})$ and Region $\mathrm{V}$ was exposed to a very low concentration of arsenic (1 ppb). The kidney cancer mortality rate ratio (RR) started to increase for Region II 10 years after exposure to a high 
concentration of arsenic compared to Region V. Mortality RRs in women and men were 2.9 and 4.4 respectively (C. Steinmaus et al., 2009).

Inorganic arsenic and its metabolites have also been shown to target the brain. Arsenic is capable of crossing the blood brain barrier and causes neurotoxicity in humans (Tolins, Ruchirawat, \& Landrigan, 2014). In an epidemiological study done in China, it was found that children who are exposed to a high level of arsenic have a lower IQ compared to an unexposed population (J. Dong \& Su, 2009). Chronic exposure to low levels of arsenic have also been linked to multiple brain related diseases. For example, individuals exposed to low concentrations of arsenic (2 ppb - $10 \mathrm{ppb})$ long term, suffered mental related illness such as depression (Tyler \& Allan, 2014). In vitro and animal studies suggest that the potential mechanisms of arsenic-induced neurotoxicity involve oxidative stress and generation of reactive oxygen species. Sun and colleagues found that chronic exposure to arsenic induces the endoplasmic reticulum (ER) stress response in the rat brain. Furthermore, long term exposure to arsenic also activates the protein kinase-like ER kinase (PERK), the eukaryotic translation initiation factor 2 subunit $\alpha(\mathrm{eIF} 2 \alpha)$, and the modulation activation of activating transcription factor 2 (ATF4). This leads to the upregulation of ER stress protein, $\mathrm{C} / \mathrm{EBP}$ homologous protein $(\mathrm{CHOP})$, and induction of neuronal apoptosis in the rat hippocampus. Lastly, an increase in homocysteine levels were observed in rat serum and brain after chronic arsenic exposure. High levels of homocysteine have been associated with oxidative stress and the induction of free radicals, leading to neuronal cell death and decline in cognitive function (Sun et al., 2017). 
In an in vitro experimental model, it was found that arsenic exposure induced cortical neuron apoptosis through the activation of c-Jun N-terminal protein kinase (JNK) and p38 mitogen activated-protein kinase (Namgung \& Xia, 2000). In a cohort study in Bangladesh, Rahman and colleagues found that prenatal arsenic exposure can potentially cause a decrease in overall birth weight of offspring (Rahman et al., 2009). An experimental animal model revealed that arsenic exposure is associated with disruption of placental morphogenesis, spontaneous abortion and low fecundity in pregnant mice (He et al., 2007). Arsenic exposure in pregnant rats induced congenital heath defects and heart malformation during embryogenesis (Y. Lin et al., 2018). An epidemiological study in Bangladesh revealed that arsenic exposure may be associated with increased fetal loss and neonatal death in areas with high levels of arsenic (Mostofa et al., 2018).

Arsenic exposure has also been linked to diabetes mellitus (DM) (Sung, Huang, Guo, \& $\mathrm{Su}, 2015)$. Specifically, arsenic biotransformation was strongly associated with an increased incidence of diabetes development. Increased levels of arsenic-associated metabolites dimethylarsinate (DMA) and a decrease in monomethylarsonate (MMA) have been associated with gestational diabetes (E. Lee et al., 2015). In an experimental animal model, arsenic exposure impaired glucose tolerance but did not result in insulin resistance in mice. Furthermore, arsenic exposure did not cause the destruction of pancreatic beta cells $(\beta$-cells). However, arsenic impaired glucose metabolism through the alteration of $\beta$ -cells function (Parsons et al., 2017). Arsenic also targets the heart. It has been reported that arsenic exposure is associated with cardiovascular disease (Moon, Exposure, \& 
Review, 2013). In vitro studies using rat aorta vascular smooth muscle cells (VSMCs) and human VSMCs demonstrated that inorganic arsenic exposure is associated with the production of ROS and nitric oxide (NO), leading to increased expression of genes associated with VSMCs proliferation. Inorganic arsenic exposure increased the expression of heme oxygenase-1 (HO-1), monocyte chemoattractant protein-1 (MCP-1) and interlurkin-6 (IL-6) in rat and human VSMCs. These genes play an important role in VSMC proliferation and the pathogenesis of atherosclerosis (P. C. Lee, Ho, \& Lee, 2005). In a case control study in China, exposure to an unsafe level of arsenic is associated with an increased risk of cardiovascular disease (Wade et al., 2015).

\subsection{Arsenic Biotransformation}

Elimination of arsenic from the body involves biotransformation of arsenic to methylated metabolites monomethylarsenous acid $\left(\mathrm{MMA}^{\mathrm{III}}\right)$, monomethylarsonic acid $\left(\mathrm{MMA}^{\mathrm{V}}\right)$, dimethylarsinous acid (DMA $\left.{ }^{\mathrm{III}}\right)$ and dimethyl arsenic acid $\left(\mathrm{DMA}^{\mathrm{V}}\right)$ that are excreted in the urine. The mechanism of arsenic biotransformation involves the conversion of pentavalent arsenic to trivalent arsenic, followed by methylation in the liver. This reaction is catalyzed by s-adenosylmethionine (SAM), glutathione (GSH) and arsenicmethyltransferase (AS3MT) (Q. Q. Wang, Wang, Jiang, Khairul, \& Naranmandura, 2017). Arsenic metabolites such as MMA and DMA are less toxic and less reactive. However, they have been associated with many diseases such as cancer, diabetes and cardiovascular disease (Kuo, Moon, Wang, Silbergeld, \& Navas-acien, 2013; E. Lee et al., 2015). 
Figure 5: Inorganic arsenic biotransformation pathway showing the reduction of arsenate to arsenite and oxidative methylation to pentavalent and trivalent forms.

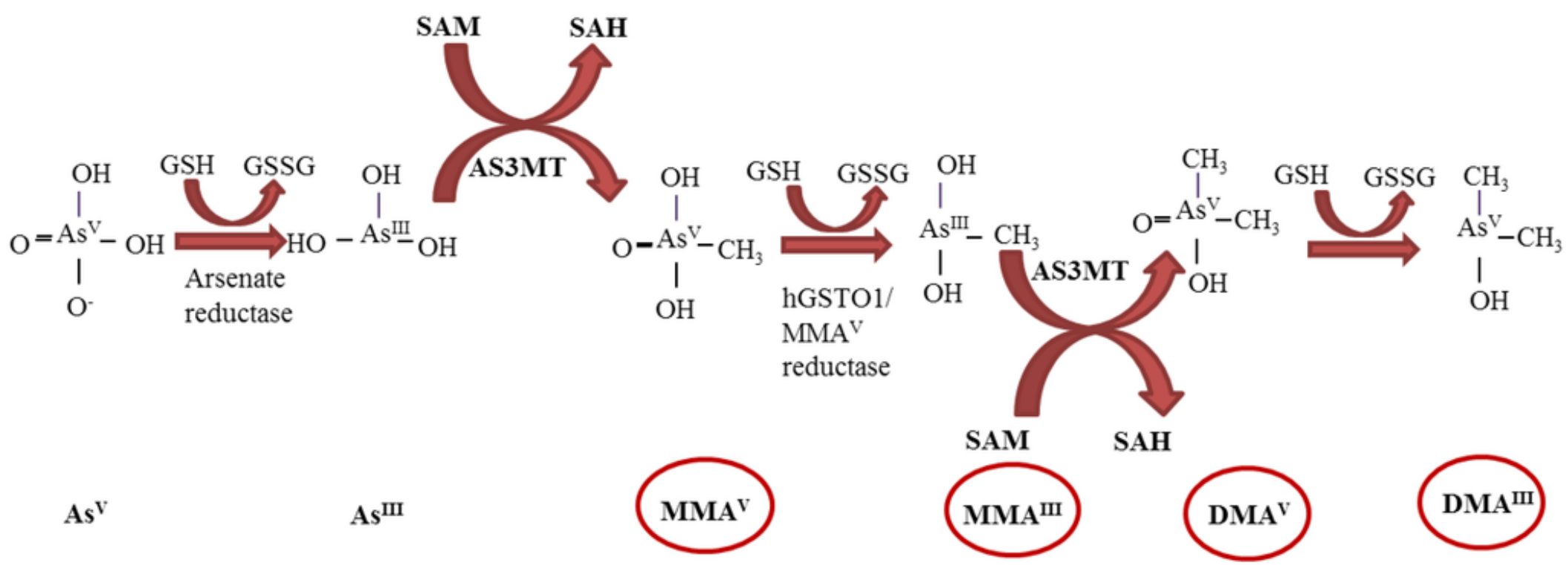

Inorganic arsenic can be found in a trivalent $\left(\mathrm{As}^{\mathrm{III}}\right)$ and a pentavalent $\left(\mathrm{As}^{\mathrm{V}}\right)$ forms. Arsenic biotransformation involves the reduction of $\mathrm{As}^{\mathrm{V}}$ to $\mathrm{As}{ }^{\mathrm{III}}$ followed by arsenic methylation by the action of s-adenosylmethionine (SAM), glutathione, and arsenicmethyltransferase (AS3MT) (Faita, Cori, Bianchi, \& Andreassi, 2013). 


\subsection{Arsenic Targets Diverse Organs}

Arsenic exposure has been shown to target diverse organs such as the heart, liver, kidneys, bladder, skin, lymph nodes, brain and lungs (Kuo et al., 2013; Liu \& Waalkes, 2008). It has been reported that arsenic exposure impairs lung function, leading to respiratory diseases (Parvez et al., 2013). Kozul and colleagues have demonstrated that arsenic exposure altered the expression of genes involved in the immune response such as toll like receptors, interleukin $1 \beta$, interleukin 1 receptors, cytokines and cytokine receptors in mouse lungs (Kozul, Hampton, et al., 2009)

\subsection{Lung Anatomy and Physiology}

Lungs are organs that play an important role in the respiratory system. The primary function of the lung is gas exchange. The respiratory system is divided into the upper and lower respiratory tracts. The upper respiratory tract includes the nasal cavity, pharynx and larynx. The trachea, bronchi and the lungs constitute the lower respiratory tract. It has been reported that the lung is a target of arsenic toxicity. Studies show that arsenic exposure negatively impacts lung function in humans and animals (Parvez et al., 2013; Ramsey, Bosco, et al., 2013).

\subsection{Arsenic Associated Lung Disease}

Exposure to arsenic has been linked to various lung diseases such as obstructive pulmonary disease (COPD), chronic bronchitis, lung cancer, respiratory symptoms, and 
bronchiectasis. For example, in a population-based cohort study of 20,033 adults in Bangladesh, chronic arsenic exposure was found to be associated with impaired lung function. They reported a decrease in forced vital capacity (FVC), which is indicative of impaired lung function (Parvez et al., 2013). In another population-based study in India, a decline in lung function was observed in people with arsenic-related skin lesions. Forced expiratory volume in one second $\left(\mathrm{FEV}_{1}\right)$ and $\mathrm{FVC}$ were reduced by $256.2 \mathrm{ml}$ and $287.8 \mathrm{ml}$ respectively. Shortness of breath was observed in smokers and morning cough in nonsmokers (N. Ghosh et al., 2005). Lung cancer is the leading cause of cancer death in the United States and other countries (Nichols, Saunders, \& Knollmann, 2012). Arsenic exposure has been associated with increased lung cancer incidence (H.-R., N.-S., H., \& R.R., 2004).

Putila and colleagues found a strong association between arsenic exposure and an increased lung cancer incidence in many parts of the United States (Putila \& Guo, 2011). There are several mechanisms associated with arsenic-induced carcinogenesis in the lungs including arsenic biotransformation, arsenic-induced induced generation of ROS, arsenic-induced alteration of DNA repair pathways, arsenic-induced genomic and chromosomal abnormalities, arsenic-induced epigenetic changes, and arsenic-induced epithelial- tomesenchymal transition (EMT). Furthermore, many studies have shown that arsenic modulates several cell signaling pathways associated with cancer such as the epidermal growth factor receptor (EGFR) pathway, the phosphoinositide-3-kinase B/Akt (PI3K/Akt), and mammalian target of rapamycin (mTOR) pathway, the erythroid-derived factor 2related factor 2 (NRF2) pathway, the Janus kinase (JAK)-signal transducers and activators 
of transcription (JAK/STAT) pathway, and the nuclear factor (FN)-kappa B (NF- $\mathrm{B})$ pathway (Cheng et al., 2004; Huang et al., 2015; Hubaux et al., 2013; Lau, Whitman, Jaramillo, \& Zhang, 2013; S. Wei, Zhang, \& Tao, 2019; D.-D. Wu et al., 2017).

In a cross-sectional study in Bangladesh, excess levels of Clara cell protein CC16 was found in the urine and serum samples of individuals who were exposed to a high concentration of arsenic in drinking water $(0.1-761 \mathrm{ppb})$. Clara cell protein $\mathrm{CC} 16$ is considered a biomarker for lung function and respiratory illness (Parvez et al., 2008). In an epidemiological study, Smith and colleagues found that pre-natal and post-natal arsenic exposure is associated with increased respiratory symptoms (Allan H. Smith et al., 2013). It has been shown that arsenic exposure along with a high fat diet resulted in the hypertrophy of lung tissue, leading to lung injury. Furthermore, it was found that a high fat diet enhances arsenic-induced lung injury through the generation of reactive oxygen species and alteration of antioxidant defense mechanisms (Hemmati, Alboghobeish, \& Ahangarpour, 2018).

Recently, numerous studies have also linked arsenic exposure to increased respiratory tract infections. In an epidemiological study, Rahman and colleagues found that pre-natal arsenic exposure is linked to increased childhood morbidity due to infectious diseases (Rahman, Vahter, Ekström, \& Persson, 2011). In a laboratory animal model, it has been shown that arsenic exposure $(0.038$ and $0.38 \mathrm{ppm})$ altered T-cell cytokine secretion and differentiation, leading to suppression of the immune system. Arsenic exposure enhanced the population of $\mathrm{CD}^{+}$cells and suppressed $\mathrm{T}$ helper cells associated cytokine secretion, 
leading to increased susceptibility to Mycobacterium fortuitum in mice (Gera et al., 2017). Arsenic exposure has also been linked to an increased incidence of pneumonia in children in Bangladesh (George et al., 2015). In an epidemiological study, arsenic exposure was shown to inhibit $\mathrm{T}$ cells proliferation and interleukin-2 (IL-2) secretion, leading to immune suppression in children (Luna et al., 2006). Furthermore, in utero arsenic exposure has been associated with alteration of $\mathrm{T}$ cells in cord blood and aberrant DNA methylation in cord blood (Kile et al., 2014). Smith and colleagues demonstrated that childhood exposure to arsenic resulted in increased mortality from lung cancer and bronchiectasis later in adulthood (Allan H. Smith et al., 2006).

\subsection{Arsenic Enhances Influenza Virus Infection}

Exposure to toxic metals has been associated with enhanced susceptibility to infectious diseases. Specifically, exposure to environmental contaminants such as arsenic, cadmium, and diesel exhaust particles (DEP) has been linked to increased susceptibility to influenza virus infection (Checconi et al., 2013; Jaspers et al., 2005; Kozul CD, 2009). Checconi and colleagues showed that exposure to environmental contaminant levels of cadmium (Cd) induced oxidative stress, leading to increased influenza virus infection in Madin-Darby canine kidney (MDCK) cells (Checconi et al., 2013). Jaspers and colleague also found that chronic exposure to diesel exhaust enhances influenza virus infection through the generation of oxidative stress in primary human bronchial epithelial cells and human lung carcinoma (A549) cells (Jaspers et al., 2005). Exposure to ozone has also been shown to increase virus infection in human nasal epithelial cells (NECs) through the disruption of protease and antiprotease balance. Respiratory proteases and antiproteases play an 
important role in the early stages of influenza virus infection by mediating the cleavage of HA to initiate virus attachment and entry (Kesic et al., 2012).

Numerous studies provided evidence that chronic exposure to arsenic disrupts the immune system, thereby increasing susceptibility to influenza virus infection in laboratory animals and humans. Kozul and colleagues demonstrated that chronic exposure to arsenic resulted in increased morbidity, increased viral titer, increased capillary leakage, decreased cytokine production, decreased oxygen saturation, decreased dendritic cell (DCs) population, and enhanced virus infection in mice (Kozul CD, 2009). In utero exposure to chronic low doses of arsenic has also been associated with enhanced influenza virus infection in mice, through an increased inflammatory response to infection. Furthermore, prenatal arsenic exposure is associated with decreased lung function and impaired lung development (Ramsey, Foong, Sly, Larcombe, \& Zosky, 2013).

\subsection{Lung Epithelial Cells}

Lung epithelial cells are the first line of defense against pathogens. They coordinate the immune defense against pathogens through secretion of antiviral factors, pro-inflammatory cytokines, reactive oxygen species, and antimicrobial peptide chemokines. Furthermore, the epithelium provides mechanical and physical barriers against pathogens by forming cell to cell junctions such as tight junctions, adherens junctions, gap junctions, and desmosomes (Vareille, Kieninger, Edwards, \& Regamey, 2011a). In addition, airway epithelial cells use a mucociliary escalator to remove pathogens and particles from the airways through the production of mucus and action of cilia (Whitsett, 2018). Airway 
epithelial cells have been shown to express various toll like receptors (TLRs) that play an important role in innate immune defense. TLRs are proteins that detect pathogens through pathogen-associated molecular patterns (PAMPs), leading to the activation of immune cells and immune response pathways (Bals \& Hiemstra, 2004). 
Figure 6: Cartoon representation of the epithelial cells acting as a barrier against respiratory viruses.

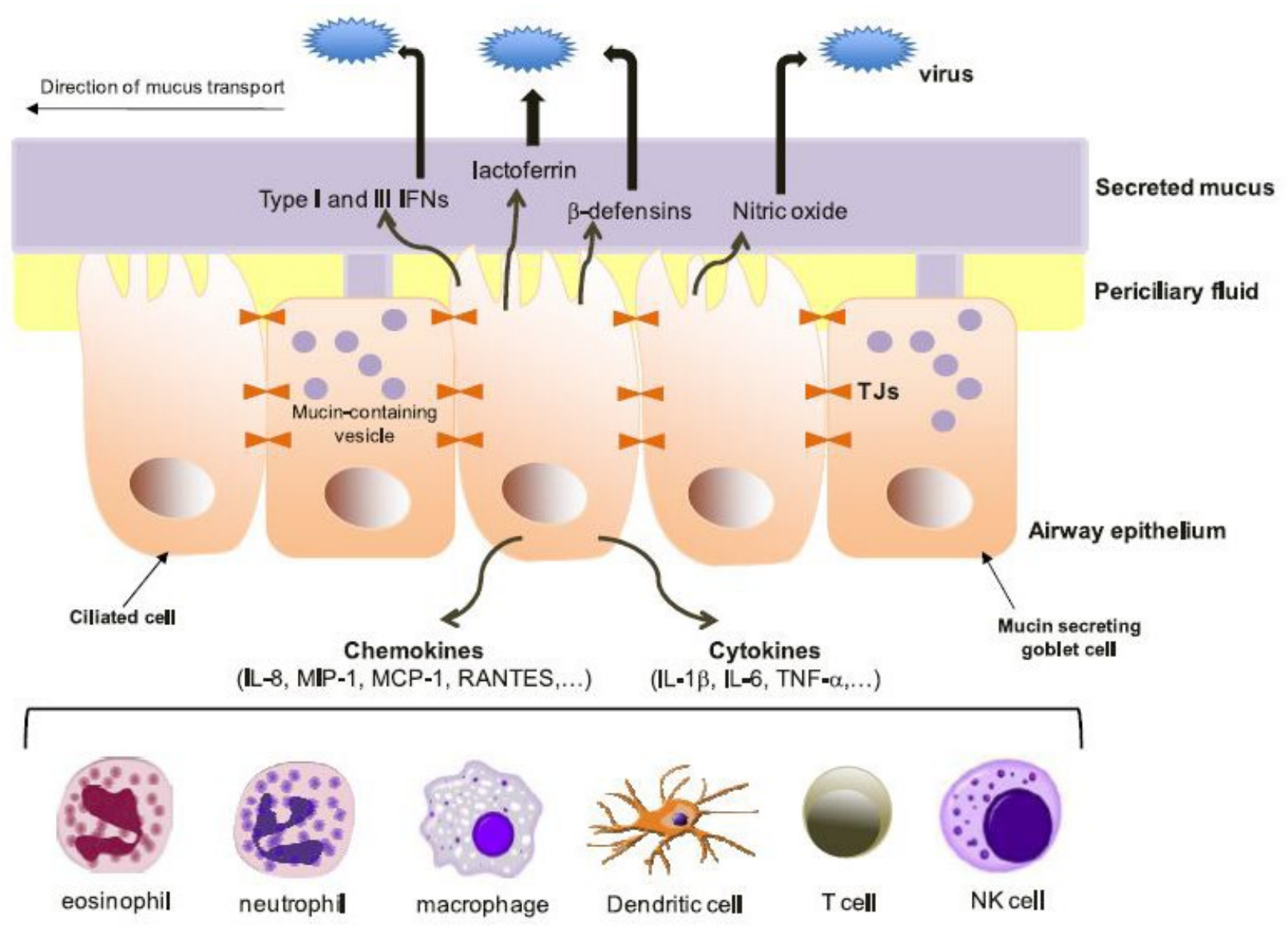

The airway epithelial cells coordinate both the innate and the adaptive immune responses through production of anti-viral factors such as type I and type III interferons (IFNs), lactoferrin, $\beta$-defensins, and nitric oxide. Epithelial cells also produce chemokines such as 
interleukin-8 (IL-8), macrophage inflammatory protein 1 (MIP-1), Regulated upon Activation, Normal T cell Expressed and Secreted (RANTES) and cytokines including, interleukin-1 $\beta$ (IL-1 $\beta$ ), interleukin-6 (IL-6), and tumor necrosis factor alpha (TNF- $\alpha$ ), leading to the recruitment and activation of the immune cells (Vareille et al., 2011a). 


\subsection{Arsenic Targets Epithelial Cells}

Arsenic has been shown to target lung epithelial cells. It has been reported that chronic exposure to arsenic has detrimental effects on epithelial cell function. The toxic effects of arsenic exposure on epithelial cells have been associated with many diseases, including respiratory diseases. Specifically, arsenic exposure has been shown to alter epithelial cell responses to pathogens, increasing susceptibility to viral and bacterial infections. Arsenic exposure has also been shown to induce the loss of epithelial cell identity, epigenetic changes, genomic instability, autophagy and lung epithelial cells (Ganapathy, Liu, Xiong, Yu, \& Makriyannis, 2018; Zeinvand-Lorestani et al., 2018; Zhao et al., 2014).

\subsection{Central Research Question and Specific Aims}

Research purpose and objectives

Epidemiological studies show that arsenic exposure through drinking water is associated with increased respiratory infections in humans. Experimental studies revealed that arsenic exposure impaired immune responses to influenza virus infection, leading to increased mortality in mice. These studies focused on the effects of arsenic on the immune system and its relation to the clinical outcomes of influenza virus infections. However, there are no studies in the literature investigating the effect of arsenic exposure on the lung epithelium. Lung epithelial cells are the primary target of influenza virus infections and there are numerous reports on the influence of virus on epithelial cells. There are numerous reports in the literature about arsenic changing epithelial cell biology such as, arsenicinduced cancer transformation in urothelial cells, and arsenic disrupting proper wound 
healing in human epithelial cells. This suggests that arsenic is capable of altering the immune response and epithelial cells response to infection. This leads to our central research question which is: Is epithelial cells targeting a part of arsenic enhancement of influenza? The following specific aims have been developed to examine this question.

Aim 1: Build models of arsenite-exposed epithelial cells in which enhancement of influenza infection will be tested.

We used MDCK, A549, and BEAS-2B cells as a model of epithelial cells to examine whether arsenic exposure increases susceptibility to influenza virus infection. We showed that MDCK, A549, and BEAS-2B demonstrated enhanced influenza infection after a longterm exposure to arsenite. The goal of this aim was to establish whether arsenic exposure enhances influenza virus infection in epithelial cells.

Aim 2: Establish the stage of infection impacted by arsenite exposure.

Influenza virus life cycle is divided into many stages and each stage is critical for successful viral replication. The goal of this aim was to determine if chronic arsenite exposure impacts an early stage of the influenza virus life cycle. This could give insight into the molecular mechanisms responsible for enhanced virus infection in the arsenite-exposed cells. Two submains were developed to investigate this aim.

Subaim 2A: Assess the impact of arsenite exposure on virus attachment.

Influenza virus binds to sialic acid molecules on the surface of the host cell and enters the cell via receptor mediated endocytosis. We have demonstrated that chronic exposure to 
arsenite increased influenza virus attachment in the arsenite-exposed MDCK and BEAS$2 \mathrm{~B}$ cells. The goal of this subaim was to determine if long term exposure to arsenic affects influenza virus attachment to the epithelial cells.

Subaim 2B: Assess influenza binding target in arsenite-exposed epithelial cells.

Influenza virus binds to sialic acid molecules on the surface of the host cell to enter the cell. Influenza virus binds to either $\alpha-2,6$-linked or $\alpha 2,3$-linked sialic acid on the cell surface. The goal of this subaim was to determine whether increased virus attachment is due to an increase in sialic acid molecules on the surface of epithelial cells. We have demonstrated that chronic exposure to arsenite resulted in increased $\alpha 2,3$-linked sialic acid quantity in the MDCK and BEAS-2B cells. However, chronic exposure to arsenite did not significantly change $\alpha-2,6-$ linked sialic acid quantity in MDCK or BEAS-2B cells. 


\section{CHAPTER 2: CHRONIC EXPOSURE TO ARSENITE ENHANCES INFLUENZA VIRUS INFECTION IN CULTURED CELLS}

Some text and figures in this section were adapted from: Amouzougan EA, Lira, R, \& Klimecki, WT. (2019) Journal of Applied Toxicology.

\subsection{ABSTRACT}

Arsenic is a ubiquitous environmental toxicant that has been associated with human respiratory diseases. In humans, arsenic exposure has been associated with increased risk of respiratory infection. Considering the existing epidemiological evidence and the wellestablished impact of arsenic on epithelial cell biology, we posited that the effect of arsenic exposure in epithelial cells could enhance viral infection. In this study, we characterized influenza virus A/WSN/33 (H1N1) infection in Madin-Darby Canine Kidney (MDCK) cells. We observed a 27.3-fold increase in viral matrix (M2) protein (24h post-infection (p.i.)), a 1.35-fold increase in viral mRNA levels, and a $126 \%$ increase in plaque area in arsenite-exposed MDCK (48h p.i.). Arsenite exposure resulted in 114\% increase in virus attachment (2h p.i.) and $224 \%$ increase in $\alpha-2,3$ sialic acid content. Interestingly, chronic exposure to arsenite reduced the effect of the antiviral drug, oseltamivir in MDCK cells. This study suggests that chronic arsenite exposure, could result in enhanced influenza infection in epithelial cells, and that it may interfere with antiviral therapy.

\subsection{INTRODUCTION}

Arsenic, a common contaminant in drinking water worldwide, is considered one of the most dangerous environmental toxicants (Minatel et al., 2018; Shen et al., 2013). High 
levels of arsenic in groundwater have been found in many countries including the United States, with millions of people exposed to arsenic levels above the U.S. Environmental Protection Agency drinking water threshold (Allan H Smith et al., 2000). Chronic exposure to inorganic arsenic via drinking water is associated with increased risk for various human cancers, as well as diseases of myriad body systems that include cardiovascular, neurologic, endocrine, integumentary, and respiratory (García-Esquinas et al., 2013; Hong, Song, \& Chung, 2014; Martinez et al., 2011; Putila \& Guo, 2011; A. H. Smith et al., 1992; Allan H. Smith et al., 2006; Tyler \& Allan, 2014). Focusing on the respiratory system, studies in arsenic-exposed populations in northern Chile documented increased risk for early death from lung cancer and obstructive pulmonary disease (Allan H. Smith et al., 2018; Allan H Smith, Goycolea, Haque, \& Biggs, 1998). Furthermore, high levels of arsenic exposure from drinking water in Bangladesh has been linked to dyspnea and pneumonia (George et al., 2015; Pesola et al., 2012). Additionally, studies have revealed a link between arsenic exposure and respiratory infections (Farzan et al., 2016).

Influenza A virus is a pathogen that can cause severe respiratory illnesses in humans (Fabian et al., 2008; Taubenberger \& Morens, 2008; Wong \& Yuen, 2006). Seasonal outbreaks of influenza A virus are responsible for annual epidemics and can sporadically result in significant global pandemics (Anhlan, Grundmann, Makalowski, Ludwig, \& Scholtissek, 2011; Shortridge et al., 2000; C. Wei et al., 2011). The Spanish flu (19181920), Asian flu (1957-1958), Hong Kong flu (1968-1970), Russian flu (1977-1978) and Swine Flu (2009-2010) are well-documented influenza pandemics known to have caused high global mortality in the $20^{\text {th }}$ century (Hinman \& Craven, 1978; Morens \& Fauci, 2007; 
Saunders-Hastings \& Krewski, 2016). Currently, influenza-related respiratory infections remain a major cause of global morbidity and mortality. According to the World Health Organization, global influenza infection rates range from 1-15\% every year, resulting in 35 million hospitalizations and approximately 250,000 - 500,000 deaths (WHO, 2011; Fischer et al., 2014). Several factors can predispose people to influenza virus infection. Age extremes, pregnancy, and pre-existing medical conditions can increase incidence and severity of influenza infection (Kunisaki \& Janoff, 2009; Littauer et al., 2017; Peltola, Ziegler, \& Ruuskanen, 2003; Ruf \& Knuf, 2014; Whitley \& Monto, 2006).

Interestingly, current evidence supports a role for arsenic exposure as a potential predisposing factor for influenza infection and severity. Farzan and colleagues provided evidence that pre-natal arsenic exposure may increase the incidence of respiratory infections in U.S. children (Farzan et al., 2016). Furthermore, Kozul et al. demonstrated that arsenic exposure alters antiviral immune responses and worsens the outcome of influenza A (H1N1) virus infection in arsenite-exposed mice (Kozul, Ely, et al., 2009). In mice, chronic exposure to arsenic has also been shown to induce pulmonary gene expression changes associated with impaired innate immune function (Kozul et al., 2008; Kozul, Hampton, et al., 2009). In addition, Ramsey and colleagues provided evidence that in utero and postnatal exposure to arsenic in mice resulted in impaired lung function, increased inflammatory response, and increased influenza A (H3N1) viral titer (Ramsey, Foong, et al., 2013). 
Lung epithelial cells act as a physical barrier against infections (Vareille, Kieninger, Edwards, \& Regamey, 2011b) and as immune system cells, secreting anti-viral factors to coordinate both the innate and adaptive immune response to infections (Ibricevic et al., 2006; Maelfait et al., 2016; Seo \& Webster, 2002; Stegemann-Koniszewski et al., 2016; Swamy, Jamora, Havran, \& Hayday, 2010; Vanleuven, Ridenhour, Gonzalez, Miller, \& Miura, 2017). Several studies have demonstrated that damage to the lung epithelial barrier can lead to increased viral and bacterial infection (Golovkine et al., 2016; Short et al., 2016; Sufiawati \& Tugizov, 2014). Evidence suggests that exposure to pollutants, including arsenic, disrupts epithelial tight junctions, leading to impaired immune response (CalderónGarciduenãs et al., 2015; Sherwood, Liguori, et al., 2013; Sherwood, Clark Lantz, \& Boitano, 2013). Additionally, arsenic exposure has been shown to disrupt proper wound healing in human airway epithelial cells (16HBE14o) through the alteration of MMP9/TIMP-1 ratio (Olsen et al. 2008). Evidence for general perturbation of epithelial cell biology by arsenic includes studies demonstrating that arsenic exposure causes loss of epithelial identity, induction of malignant transformation, and the induction of pseudohypoxia in an $\underline{\text { in vitro }}$ model of human bronchial epithelial cells (Zhao et al., 2014). Taken together, these studies show that arsenic has the ability to broadly perturb epithelial cell biology. Based on these studies, we hypothesized that, through non-cytotoxic perturbation of epithelial cell biology, arsenic exposure could render epithelial cells more conducive to influenza virus infection. In this study, we characterized influenza A virus A/WSN/33 (H1N1) infection in arsenite-exposed Madin-Darby Canine Kidney (MDCK) epithelial cells. This study lends insight into novel molecular mechanisms through which arsenic may increase the risk or severity of influenza virus infection in exposed human populations. 


\subsection{METHOD}

\section{Cell culture}

MDCK cells were obtained from Dr. Jun Wang at the University of Arizona and cultured in Dulbecco's Modified Eagle Medium (DMEM; Gibco, Grand Island, NY) media supplemented with 10\% fetal bovine serum (FBS; Gibco, Grand Island, NY), and 100 units/ml of Antibiotic-Antimycotic (ABAM; ThermoFisher Scientific, Waltham, MA). MDCK were seeded in $75 \mathrm{~cm}^{2}$ Falcon tissue culture treated flasks (ThermoFisher Scientific, Waltham, MA) and were passaged every 3 days at $80-90 \%$ confluence, with reseeding performed at a density ratio of 1:10. Cells were maintained in a humidified incubator at $37{ }^{\circ} \mathrm{C}$ and $5 \% \mathrm{CO}_{2}$ atmosphere. The identity of MDCK cells was confirmed through the use of genetic analysis using short-tandem repeat genotyping at multiple loci.

\section{Arsenite Exposure}

Arsenite-exposed cells were maintained on the same passage schedule as mentioned above, but were continuously cultured in media containing $1 \mu \mathrm{M}$ sodium arenite. Arseniteunexposed cells used in this study were passage matched to arsenite-exposed cells.

\section{Cell viability Assay}

MDCK cells were plated into a clear 96-well microplate at a density of 4,000 cells/well in DMEM supplemented with 10\% FBS and 100 units/ml of antibiotic-antimycotic (ABAM; Fisher Scientific). After 24 hours, cells were treated with various concentrations of sodium 
arsenite $(0-100 \mu \mathrm{M})$ and incubated in a humidified incubator at $37{ }^{\circ} \mathrm{C}$ and $5 \% \mathrm{CO}_{2}$ atmosphere for 72 hours. Cell viability was measured using the CellTiter 96 Aqueous One Solution Cell Proliferation Assay (MTS, Promega, Madison, WI) according to manufacturer protocols. After 72 hours incubation with sodium arsenite, $20 \mu$ of MTS/PMS solution was added to each well, and the plate was further incubated in a humidified incubator at $37{ }^{\circ} \mathrm{C}$ and $5 \% \mathrm{CO}_{2}$ atmosphere for 1.5 hours. The conversion of tetrazolium to formazan was measured at optical absorbance-490 nm using a Synergy HTX Multi-Mode Microplate Reader (BioTek, Winooski, VT).

\section{Virus and virus Amplification}

Influenza A virus strain, A/WSN/33 (H1N1) was obtained from Dr. Jun Wang at the University of Arizona. The initial virus stocks were amplified in MDCK cells cultured in DMEM media supplemented with $0.5 \%$ bovine serum albumin (BSA; Sigma Aldrich, St. Louis, MO) and $2 \mu \mathrm{g} / \mathrm{ml} \mathrm{N}$-acetyl trypsin (Sigma Aldrich, St. Louis, MO). Culture supernatant was collected two days post-infection and centrifuged for 30 minutes at 3000 rpm to remove cell debris. Plaque assays were used to determine the viral titers using MDCK cells. Viral strain identity was confirmed by DNA sequencing of RT-PCRamplified diagnostic viral amplicons.

\section{Immunoblotting}

Cells were seeded at a density of 500,000 cells/well in 6-well plates. Forty-eight hours after seeding, cells were infected with influenza A/WSN/33 (H1N1) virus (1000 pfu) in N-acetyl trypsin $(2 \mu \mathrm{g} / \mathrm{ml})$ containing media. The plate was further incubated at $37^{\circ} \mathrm{C}$ and $5 \% \mathrm{CO}_{2}$ 
atmosphere for additional 24 hours. After incubation, cells were washed twice with cold PBS and lysed in NP40 Cells Lysis Buffer [(pH 7.4), $150 \mathrm{mM} \mathrm{NaCl,} 50 \mathrm{mM}$ EDTA, 50 $\mathrm{mM}$ Tris, and 1\% Nonidet P40 (NP40)]. Cell lysis buffer was supplemented with 1x MSSAFE Protease and Phosphatase Inhibitor (MilliporeSigma, Darmstadt, Germany). Samples were sonicated and subjected to centrifugation at 12,000 rpm for 20 minutes at 4 ${ }^{\circ} \mathrm{C}$ to remove cell debris. After sonication, total protein in the supernatant was quantified using the Pierce $660 \mathrm{~nm}$ Protein Assay Reagent (ThermoFisher Scientific, Waltham, MA). Samples were subject to sodium dodecyl sulfate polyacrylamide gel electrophoresis (SDSPAGE), and separated proteins were blotted on nitrocellulose membrane (Bio-Rad, Hercules, CA). Anti-GAPDH (Santa Cruz Biotechnology, Dallas, TX), and Anti-M2 (GeneTex, Irvine, CA) antibodies were used for protein detection. All horseradish peroxidase-secondary antibodies were purchased from Santa Cruz Biotechnology (Dallas, TX). Anti-GAPDH and Anti-M2 antibodies were used at a concentration of 1:5000 and 1:1000 respectively. Secondary antibodies were used at a concentration of 1:5000. AntiGAPDH antibody was used as loading control for all western blots. Immunoblots were developed using ECL Western Blotting Substrate (ThermoFisher Scientific) and detected using GeneGnome5 imaging system (Syngene, Frederick, MD).

Quantitative Real-Time PCR (qRT-PCR)

Arsenite-unexposed and arsenite-exposed cells were seeded at a density of 500,000 cells/well in a standard 6-well plate. Forty-eight hours after seeding, cells were infected with influenza A/WSN/33 (H1N1) virus (1000 pfu) in N-acetyl trypsin $(2 \mu \mathrm{g} / \mathrm{ml})$ containing media. The plate was incubated at $37{ }^{\circ} \mathrm{C}$ and $5 \% \mathrm{CO}_{2}$ atmosphere for another 48 hours. Total RNA was isolated from cells using RNeasy Mini Kit (Qiagen, Valencia, 
CA), and a NanoDrop 2000 spectrophotometer (ThermoFisher Scientific, Waltham, MA) was used to determine RNA concentration. Quantitative real-time PCR was performed using TaqMan RNA-to-CT-1-Step Kit (ThermoFisher Scientific, Waltham, MA) in a StepOnePlus Real Time PCR system (Life Technologies; Grand Island, NY), according to the manufacturer guidelines. Total cellular RNA used was 20 ng per reaction. Dog glyceraldehyde-3-phosphate dehydrogenase copy number was measured in the cDNA as an internal control with the following TaqMan assay ID: gapdh (Cf04419463_gH). All Taqman Gene Expression assays were purchased from Thermofisher Scientific (Waltham, MA). Influenza A matrix gene mRNA levels were measured using previously published primer and probe sequences (Centres et al., 2017; Yoshida, Sakoda, Endo, Motoshima, \& Yoshino, 2011). The sequences of primers and hybridization probe targeting the matrix gene were as follows: MP-39-67Forward: 5'CCMAGGTCGAAACGTAYGTTCTCTCTATC $\quad$-3' MP-183-153Reverse: 5'TGACAGRATYGGTCTTGTCTTTAGCCAYTCCA-3', MP-96-75 ProbeAs 5'-(FAM)ATYTCGGCTTTGAGGGGGCCTC-(MGB)-3'. The matrix gene primers and probe were custom-made by Thermofisher Scientific (Waltham, MA). The $2^{-\Delta \Delta_{C}}$ Tethod was used to analyze qRT-PCR results as previously described by Schmittgen et al. (Livak \& Schmittgen, 2001) using StepOne Software v2.3 from Applied Biosystems (Waltham, MA).

Plaque Assay 
MDCK cells were grown to $90 \%-100 \%$ confluence then infected with 100 plaque forming units (pfu) of influenza A/WSN/33 (H1N1) virus in $0.5 \mathrm{ml}$ of culture media containing $0.5 \%$ BSA for 1 hour at $4{ }^{\circ} \mathrm{C}$ followed by 1 hour incubation at $37^{\circ} \mathrm{C}$. After incubation, the virus was removed, and cells were washed with PBS. 1.2\% Avicel mixed with 1x DMEM media containing $2 \mu \mathrm{g} / \mathrm{ml} \mathrm{N}$-acetyl trypsin (Sigma Aldrich, St. Louis, MO) was added to the cells. Cells were incubated at $37{ }^{\circ} \mathrm{C}$ for 48 hours to allow plaque formation. Plaques were visualized as unstained foci using crystal violet staining of live cells (Sigma Aldrich; $0.1 \%$ crystal violet in $20 \%$ methanol). A tripod-mounted Nikon D3200 DSLR was used to capture images. Images were captured at a shutter speed of $1 / 30$ second with the $(50 \mathrm{~mm})$ lens aperture set to f/16. ImageJ version $1.50 \mathrm{i}(\mathrm{NIH}$, Bethesda, MD) was used to process and analyze images. Image J analysis used macros to automate image processing/analysis commands, and all images were processed/analyzed using a single set of parameters. Briefly, "image" was selected and "otshu threshold" was applied to convert the image to a binary image. The background was set to white by inverting the image, and pixel size was set to "250-infinity".

Antiviral Drug Response Assay

Plaque assays were carried out as previously described. Briefly, MDCK cells were inoculated with $100 \mathrm{pfu}$ of influenza A/WSN/33 (H1N1) virus. The inoculation was removed, and cells were incubated with Avicel media containing $2 \mu \mathrm{g} / \mathrm{ml} \mathrm{N}$-acetyl trypsin (Sigma Aldrich, St. Louis, MO) and $100 \mathrm{nM}$ oseltamivir carboxulate (Sigma Aldrich, St. Louis, MO). Cells were incubated at $37{ }^{\circ} \mathrm{C}$ for 48 hours to allow plaque formation and plaques were visualized as described in the previous section. 


\section{Virus Attachment Assay}

Arsenite-unexposed and arsenite-exposed cells were washed twice PBS (100,000 cells/tube) and were infected with influenza A/WSN/33 (H1N1) virus $\left(4 \times 10^{6} \mathrm{pfu}\right)$ at $4{ }^{\circ} \mathrm{C}$, and then maintained at $4{ }^{\circ} \mathrm{C}$ for 2 hours to allow virus attachment. After removing the virus, cells were washed with PBS and resuspended in 10\% BSA and 1\% sodium azide (Sigma Aldrich, St. Louis, MO) blocking solution for 30 minutes on ice. After the blocking solution was removed, cells were then resuspended in 3\% BSA diluted in PBS containing 1:10 dilution of influenza A anti-NP monoclonal antibody conjugated to fluorescein isothiocyanate (FITC) (ThermoFisher Scientific, Waltham, MA) or 1:10 dilution of isotype control monoclonal antibody conjugated to FITC (ThermoFisher Scientific, Waltham, MA) for 1 hour on ice. Cells were washed twice with PBS and resuspended in 3\% BSA diluted in PBS and placed on ice. Cells were analyzed by flow cytometry using LSRII flow cytometer (BD Biosciences, San Jose, CA).

\section{Sialic Acid Quantification}

For the quantification of $\alpha 2,6$ sialic acid, arsenite-unexposed and arsenite-exposed cells were washed twice with PBS (500,000 cells/tube) and were covered with $100 \mathrm{ng} / \mathrm{ml}$ of Sambucus Nigra (SNA) lectin conjugated to cyanine-5 (Cy5) (Vector Laboratories, Burlingame, CA) for 30 minutes on ice. Cells were washed twice with PBS, resuspended in PBS and placed on ice. Cells were analyzed by flow cytometry using LSRII flow cytometer (BD Biosciences, San Jose, CA). To quantify $\alpha 2,3$ sialic acid, arseniteunexposed and arsenite-exposed cells were washed twice with PBS (500,000 cells/tube) 
and were covered with 10ng/ml of biotinylated Maackia Amurensis lectin II (MALII) (Vector Laboratories, Burlingame, CA) for 30 minutes on ice. Cells were washed with PBS, and loaded with 1:200 dilution of streptavidin (STV) conjugated to cyanine-3 (Cy3) (Thermofisher Scientific, Waltham, MA) to allow for the detection of biotinylated MALII. Cells were washed twice with PBS, resuspended in PBS and placed on ice. Cells were analyzed by flow cytometry using a LSRII flow cytometer (BD Biosciences, San Jose, CA).

\section{Statistical Analysis}

Statistical analysis was performed using GraphPad Prism version 6.07 for Windows (GraphPad Software Inc., La Jolla, CA). Analysis of RNA fold change utilized a one sample $t$-test with a null hypothesis that the measured fold change $=1.0$ (no change in RNA quantity between samples). Unpaired, two sample t-tests were used to compare mean differences between 2 groups. An alpha value threshold of 0.05 was used to determine statistical significance.

\subsection{RESULTS}

Chronic exposure to low dose of sodium arsenite $(1 \mu M)$ is not toxic to cells

We have previously demonstrated that chronic exposure to $1 \mu \mathrm{M}$ of sodium arsenite was not toxic to BEAS-2B human bronchial epithelial cells, but induced gene expression changes (Zhao \& Klimecki, 2015). To rule out differential cytotoxicity response between chronically arsenite-exposed and control MDCK cells as a confounding factor in this study, we repeated the cytotoxicity experiments in control and chronically arsenic- 
exposed MDCK. We observed similar results as our prior studies, indicating that MDCK cells in those two exposure conditions demonstrated similar cytotoxicity dose-response to 72-hour arsenic exposure (Figure 1A).

Sodium arsenite exposure increases influenza A/WSN/33 (HINI) virus protein levels and $m R N A$ quantity in infected $M D C K$

Immunoblot analysis revealed significantly increased (27.3-fold) levels of influenza A/WSN/33 (H1N1) matrix (M2) protein in the arsenite-exposed MDCK cells relative to arsenite-unexposed MDCK cells at 24 hours post-infection (Figure 1B, 1D). Influenza A virus matrix (M) mRNA quantity was measured using qRT-PCR after infection with influenza A/WSN/33 (H1N1) virus. Consistent with protein levels, qRT-PCR analysis revealed a statistically significant increase (1.35-fold) in influenza A matrix (M) mRNA quantity in the arsenite-exposed MDCK cells compared to arsenite-unexposed MDCK cells at 48 hours post infection (Figure 1C). Taken together, these results indicate that, compared to arsenite-naïve MDCK cells, chronically arsenite-exposed MDCK cells respond to influenza infection with higher production of viral protein.

Sodium arsenite exposure results in increased viral lytic damage in MDCK after infection with A/WSN/33 (H1NI)

Plaque assays were used as an orthogonal measure of MDCK response to influenza A/WSN/33 (H1N1) infection in arsenite-exposed and arsenite-unexposed MDCK cells. Forty-eight hours post-infection with 100 pfu of the virus, arsenite exposed MDCK 
demonstrated a $127 \%$ increase in total plaque area compared to identically infected, arsenite-unexposed MDCK cells (Fig 2A, 2B). Collectively, these results showed that exposure to sodium arsenite increased lytic infection by influenza A/WSN/33 (H1N1) virus in arsenite-exposed MDCK cells.

Sodium arsenite exposure decreases the effect of the anti-influenza drug, oseltamivir To evaluate the effect of arsenite exposure on response to the anti-viral drug oseltamivir, arsenite-exposed and arsenite-unexposed MDCK cells were infected with 100 pfu of influenza A/WSN/33 (H1N1) virus. After removing the inoculum, cells were incubated with $100 \mathrm{nM}$ oseltamivir for 48 hours during the evolution of plaque formation. The results revealed that the differentially increased plaque formation in infected arsenite-exposed MDCK cells was not abrogated by treatment with oseltamivir (Figure 3A-3D). Consistent with other experiments, the total plaque area in arsenite-exposed cells was significantly increased compared to arsenite-unexposed cells. Interestingly, we found that oseltamivir was 1.6 times less effective in plaque area reduction in the arsenite-exposed MDCK cells, compared to infected control cells (Figure 3D).

\section{Sodium arsenite exposure increases influenza A/WSN/33 (H1N1) virus attachment in}

\section{infected MDCK}

We used flow cytometry to assess whether chronic arsenic exposure impacted virus attachment, defined in these experiments as cellular virus quantity following exposure to virus at a constant temperature of $4{ }^{\circ} \mathrm{C}$. We observed a $114 \%$ higher $\mathrm{H} 1 \mathrm{~N} 1$ nucleoprotein fluorescent signal in arsenite-exposed MDCK cells, compared to control MDCK cells 
(Figure 4). These results suggest that chronic arsenite exposure may enhance viral attachment to target cells.

Sodium arsenite exposure increases $\alpha$-2,3-linked sialic acid quantity in MDCK

To determine the effect of arsenic exposure on cell-surface target molecules of influenza binding, we used a flow cytometric approach to quantify $\alpha-2,3$-linked and $\alpha-2,6$-linked sialic acids in control and arsenite-exposed MDCK cells. The fluorescent signal for $\alpha-2,3-$ linked sialic acid was increased by $224 \%$ in the arsenite-exposed MDCK cells compared to control MDCK cells (Figure 5). In contrast to this we did not observe a significant effect of arsenic exposure on $\alpha-2,6$-linked sialic acid quantity (Figure 5C).

\subsection{DISCUSION}

Evidence supports the potential for arsenic to have immunosuppressive activity that can affect host response to influenza virus infection (Farzan et al., 2016; Kozul, Ely, et al., 2009; Nayak, Lage, \& Kim, 2007). Arsenic has also been shown to compromise epithelial cells response to pathogens (Henderson et al., 2017). However, studies linking the effects of arsenic exposure on the influenza virus susceptibility of epithelial cells are lacking. In the present in vitro study, we aimed to explore a novel pathway through which arsenic compromises epithelial cell function, leading to increased influenza virus infection.

MDCK cells are a gold-standard as an in-vitro model of mammalian influenza virus infection, and have been shown to be the most efficient cell line for influenza virus 
propagation (Gaush \& Smith, 1968). Influenza virus binds to $\alpha$-2,6-linked and $\alpha-2,3$-linked sialic acids on the surface of cells and enters target cells through receptor mediated endocytosis (García-Sastre, 2010; Shi, Wu, Zhang, Qi, \& Gao, 2014). Studies showed that MDCK cells expressed both the $\alpha-2,6$-linked and $\alpha-2,3$-linked sialic acids, which likely makes them a good model for influenza virus research (Ilyushina et al., 2012; T Ito et al., 1997; S. C. Lin, Kappes, Chen, Lin, \& Wang, 2017). Thus, MDCK cells were used as a model of arsenic-exposed epithelial cell line in this study.

We began by assessing the impact of arsenic exposure on influenza virus mRNA and protein synthesis, which are key steps in viral pathogenesis within target cells (Dou et al., 2018; Matsuoka et al., 2013). Chronic arsenite exposure resulted in increased viral RNA expression and viral protein synthesis in arsenite-exposed MDCK cells. In lytic infections this increased production of viral proteins could lead to enhanced cell lysis. In fact we did observe increased plaque formation in virus infected cells, revealing that arsenite exposure increased lytic influenza virus infection in MDCK cells. These results are not inconsistent with previous reports showing that in vivo arsenic exposure in mice and zebrafish resulted in increased virus infection from influenza and snakehead rhabodovirus, respectively (Kozul CD, 2009; Nayak et al., 2007). In contrast to our work, authors in those studies did not focus on epithelial cell virus targets as mediators of this effect.

To elucidate points in the influenza infection process that may be impacted by arsenic exposure, we investigated the effects of arsenic exposure on virus attachment. Virus 
attachment assay revealed that chronic arsenite exposure resulted in a $114 \%$ increase in influenza virus attachment in MDCK cells (Figure 4). This result suggests arsenicmediated enhancement of influenza virus infection may be, at least in part, due to increased virus attachment to influenza virus molecular binding targets. We used microbial lectins that bind sialic acids with specific linkage to quantify $\alpha$-2,6-linked and $\alpha$-2,3-linked sialic acids in arsenite-unexposed and arsenite-exposed MDCK. Interestingly, we found a significant increase (224\%) in $\alpha$-2,3-linked sialic acid detected in arsenite-exposed MDCK compared to arsenite-unexposed MDCK (Figure 5). In contrast, there were no significant differences in the $\alpha-2,6$-linked sialic acid quantity in the arsenite-unexposed and arseniteexposed MDCK. Unexpectedly, the enhanced influenza virus attachment correlates with the increase $\alpha-2,3$-linked sialic acid expression in the arsenite-exposed MDCK. Although human influenza viruses preferentially bind to $\alpha-2,6$-linked sialic acid, they have also been shown to weakly bind to $\alpha-2,3$-linked sialic acid $(\mathrm{Xu}$, McBride, Nycholat, Paulson, \& Wilson, 2011).

Influenza virus is a major threat to public health in part due to challenges in treatment and disease prevention. Oseltamivir is one of the currently available FDA approved antiviral drugs for the treatment and prevention of the influenza virus. Oseltamivir is a neuraminidase (NA) inhibitor that blocks the release of new viral particles through inhibition of the neuraminidase enzyme. In this study, we were interested to know if treatment of MDCK cells with oseltamivir could reverse the arsenite-enhanced influenza virus infection. Surprisingly, the enhanced influenza virus infection persisted in the presence of oseltamivir. Chronic exposure to sodium arsenite resulted in increased plaque formation in oseltamivir treated virus infected cells. This study suggests that chronic 
arsenite exposure may decrease the effect of oseltamivir. Decrease in oseltamivir effectiveness due to arsenite exposure can have severe clinical implications in arsenicexposed human populations.

Our study suggests that arsenic enhancement of influenza virus infection may be mediated by increase in $\alpha 2,3$-linked sialic acid expression in MDCK cells. Furthermore, chronic arsenic exposure reduced the effect of the influenza drug, oseltamivir. Future studies will determine whether arsenic exposure has similar effects on influenza virus infection in lung relevant human epithelial cell lines.

The molecular mechanisms by which arsenic-enhanced influenza virus infection is not fully elucidated. Our data suggests that arsenite-mediated increase $\alpha-2,3$-linked sialic acid expression could be responsible for increase virus attachment, resulting in enhanced influenza A/WSN/33 (H1N1) virus infection in MDCK cells. Our study is consistent with a model in which chronic exposure of MDCK cells to sodium arsenite resulted in increased $\alpha-2,3-$ linked sialic acid, leading to enhanced influenza binding, resulting in enhanced intracellular synthesis of viral components. In developing this model we confirm previous reports that arsenic exposure increases susceptibility to influenza virus infection, and uncovered a novel potential mechanism mediating arsenic-enhanced influenza infection in vitro. However, more research is needed to discern all the potential mechanisms mediating arsenic-enhanced influenza virus infection.

\section{Conclusion:}


1. Chronic exposure of MDCK cells to sodium arsenite $(1 \mu \mathrm{M})$ at nontoxic levels increases influenza virus protein levels and mRNA quantity virus-exposed cells.

2. Sodium arsenite exposure increases plaque formation in arseniteexposed MDCK cells.

3. Sodium arsenite exposure decreases the effect of oseltamivir.

4. Sodium arsenite exposure increases virus attachment in arseniteexposed MDCK cells.

5. Sodium arsenite exposure increases $\alpha 2,3$-linked sialic acid quantity in arsenite-exposed MDCK cells.

6. Sodium arsenite exposure did not result in significant differences in $\alpha 2,6$-linked sialic acid quantity in arsenite-unexposed and arseniteexposed MDCK cells. 
Figure 7: Exposure to sodium arsenite enhanced influenza A/WSN/33 (H1N1) virus infection in MDCK cells

A

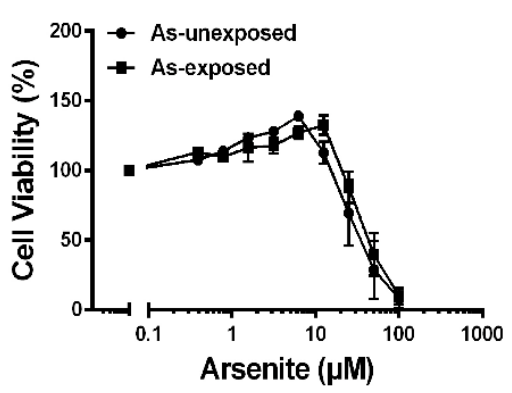

C

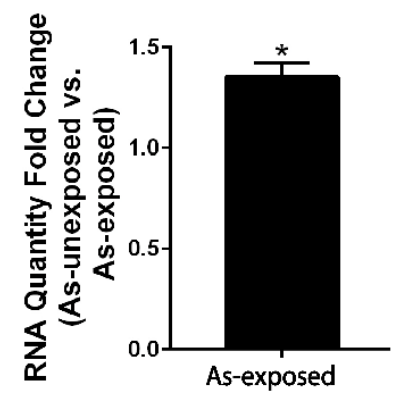

B

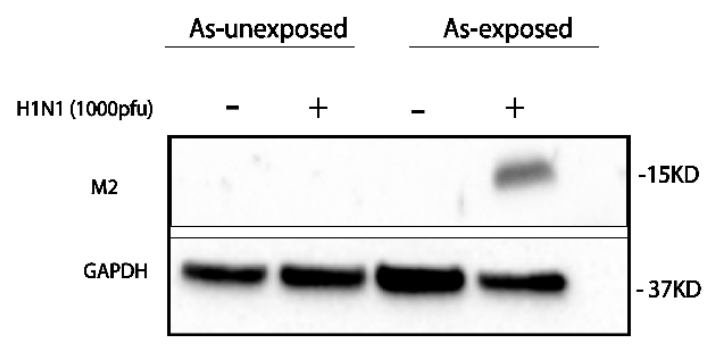

D

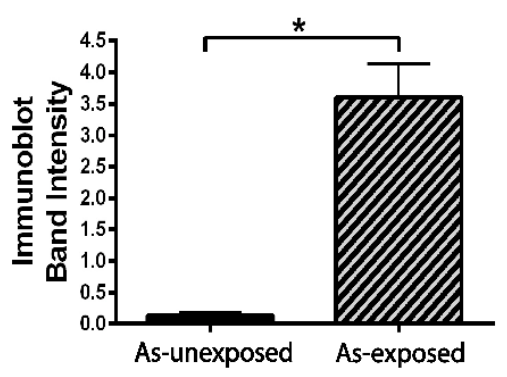

A. Cell viability in As-unexposed and As-exposed MDCK cells treated with $0-100 \mu \mathrm{M}$ sodium arsenite for $72 \mathrm{~h}$. Error bars represent means \pm standard error of the mean (SEM). $\mathrm{n}=6$ (technical replicates), data from 3 independent biological replicates. B. Representative immunoblots showing viral matrix (M2) protein levels in influenza A virus infected As-unexposed and As-exposed MDCK cells at 24h p.i. C. Viral matrix gene (M) quantity in influenza A virus infected As-unexposed and As-exposed MDCK cells at 48h p.i. Error bars represent means \pm SEM. $n=3$ independent biological replicates. ${ }^{*}=p<0.05$ (one-sample t-test referenced to fold change $=1$ ). D. Immunoblots band intensity of M2 normalized to GAPDH. Error bars represent means \pm SEM. $n=3$ independent biological replicates. ${ }^{*}=\mathrm{p}<0.05$. 
Figure 8: Sodium arsenite exposure leads to increased plaque formation in MDCK cells at $48 \mathrm{~h}$ p.i with influenza A/WSN/33 (H1N1) virus.

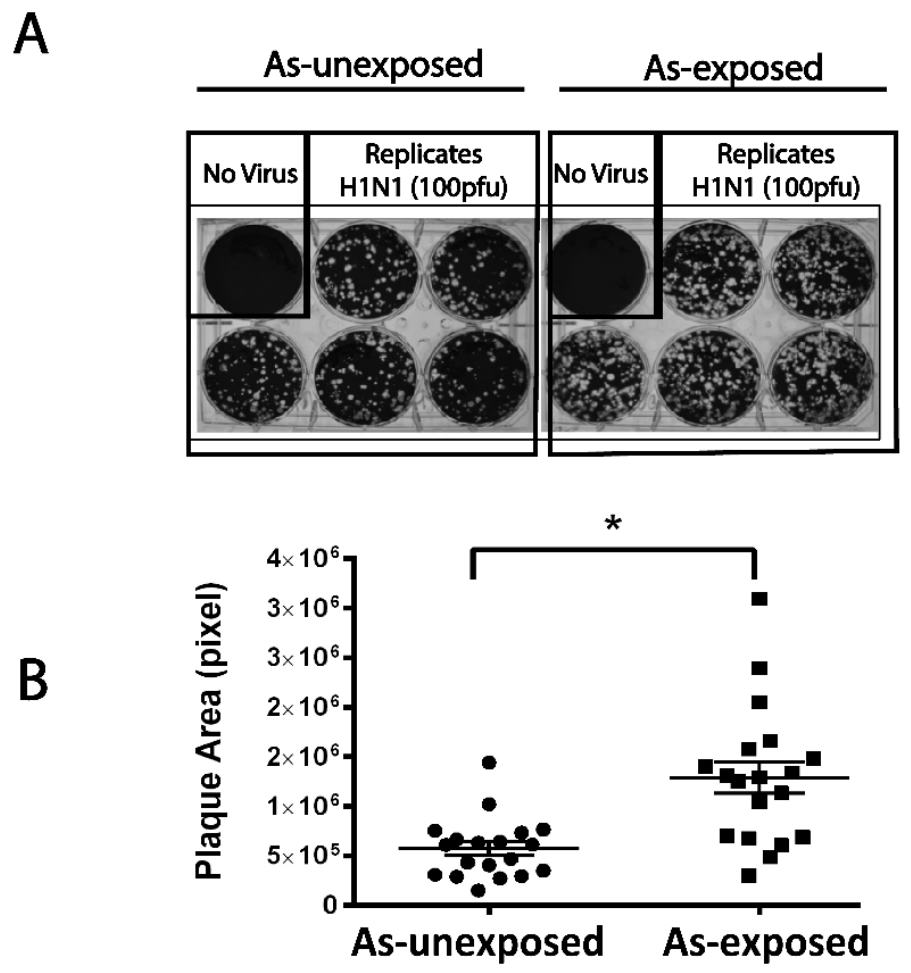

A. Plaque produced in As-unexposed and As-exposed MDCK cells at 48h p.i with influenza A/WSN/33 (H1N1) virus. B. Total plaque area in As-unexposed and As-exposed MDCK cells at 48h p.i with influenza A/WSN/33 (H1N1) virus. Error bars represent means \pm SEM. $n=5$ (technical replicates), data from 19 independent biological replicates. Unpaired t-test with Welch's correction was used to determine statistical significance between influenza A/WSN/33 (H1N1) infected As-unexposed and As-exposed MDCK cells. $*=p<0.05$. 
Figure 9: Sodium arsenite reduced the efficacy of anti-viral drug oseltamivir.

A

As-unexposed $\frac{\text { As-unexposed }}{\text { Oseltamivir }(100 \mathrm{nM})}$

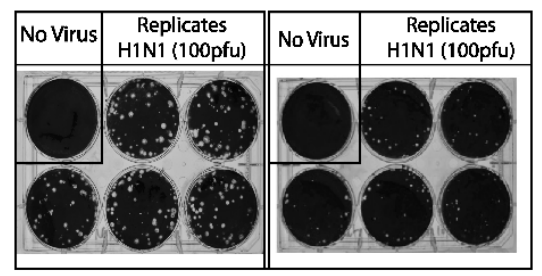

C

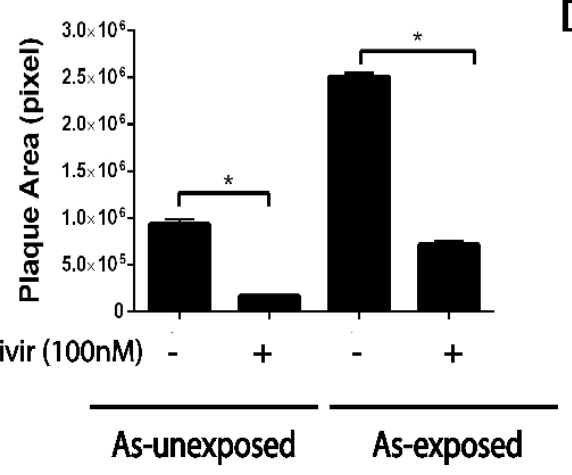

B
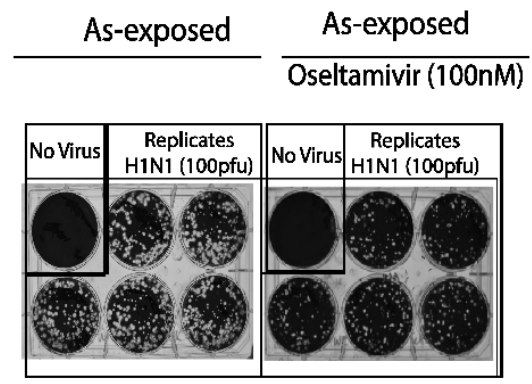

D

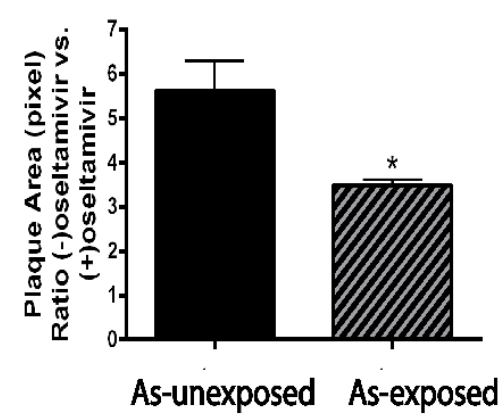

A. Plaque produced in As-unexposed and As-exposed MDCK cells at 48h p.i with influenza A/WSN/33 (H1N1) virus. B. Plaque produced in As-unexposed and As-exposed MDCK cells at 48h p.i with influenza A/WSN/33 (H1N1) virus co-treated with 100nM Oseltamivir. C. Total plaque area in As-unexposed and As-exposed MDCK cells at 48h p.i with influenza A/WSN/33 (H1N1) virus. Error bars represent means \pm SEM. $n=3$ independent biological replicates. $*=p<0.05$ D. Total plaque area ratio of infected cells without oseltamivir vs. infected cells with oseltamivir in As-unexposed and As-exposed MDCK cells at 48 h p.i. Error bars represent means \pm SEM. $n=3$ independent biological replicates. ${ }^{*}=\mathrm{p}<0.05$. 
Figure 10: Exposure to sodium arsenite enhanced influenza A/WSN/33 (H1N1) virus attachment in MDCK cells.

A

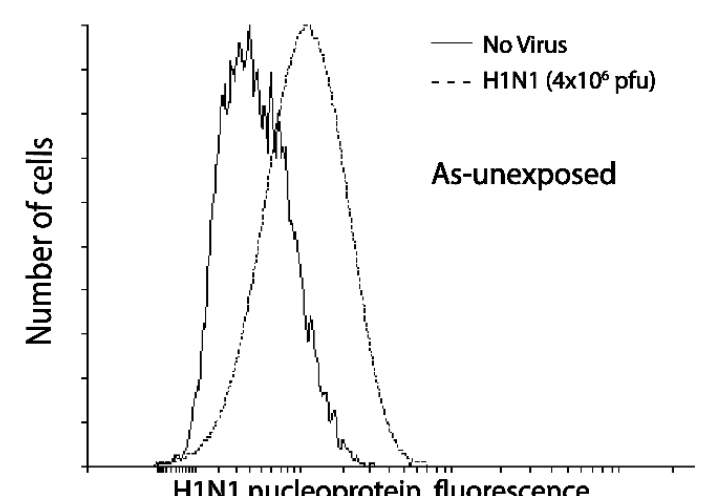

B

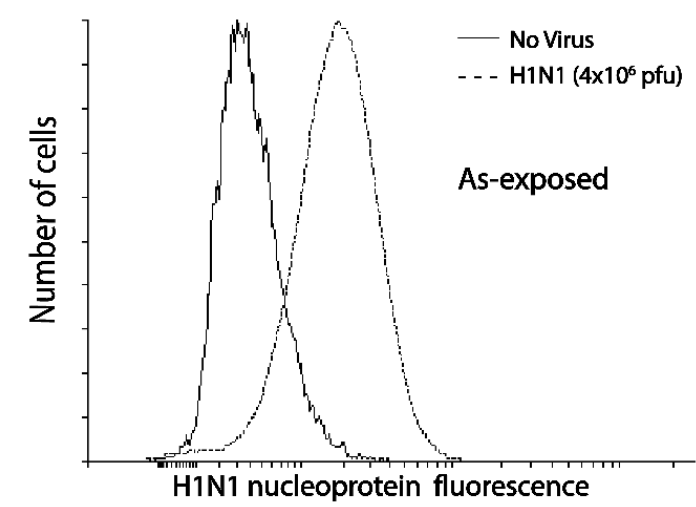

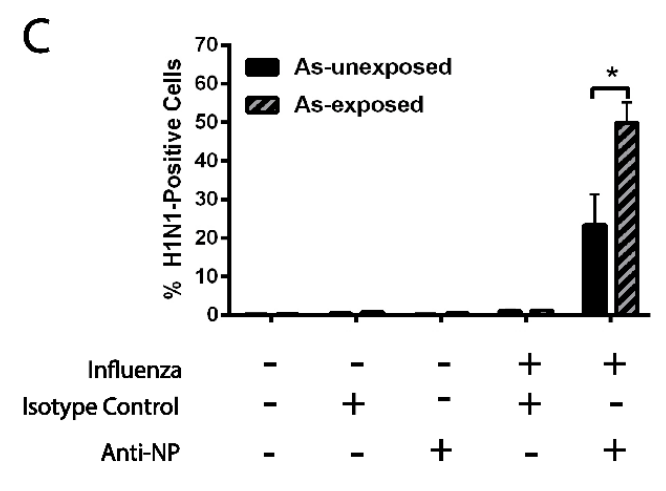

A. A representative cytometric histogram showing H1N1 nucleoprotein fluorescence signal (x-axis) in As-unexposed MDCK cells (solid line represents uninfected control population and dashed line represents infected population). B. A representative cytometric histogram showing H1N1 nucleoprotein fluorescence signal (on the x-axis) in As-exposed MDCK cells (solid line represents uninfected control population and dashed line represents infected population). C. Percentage of H1N1-positive cells in As-unexposed and Asexposed MDCK cells at $2 \mathrm{~h}$ p.i. Error bars represent means \pm SEM. $\mathrm{n}=3$ independent biological replicates. $*=p<0.05$ 
Figure 11: Exposure to sodium arsenite increases $\alpha-2,3$-linked sialic acid quantity in MDCK cells.

A

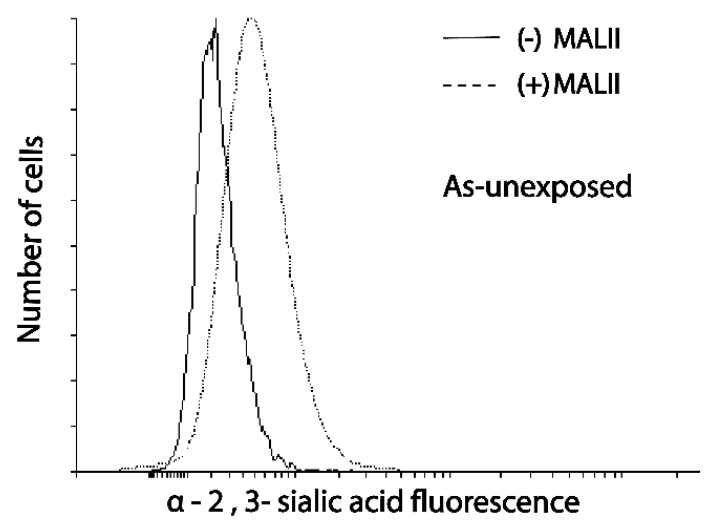

C

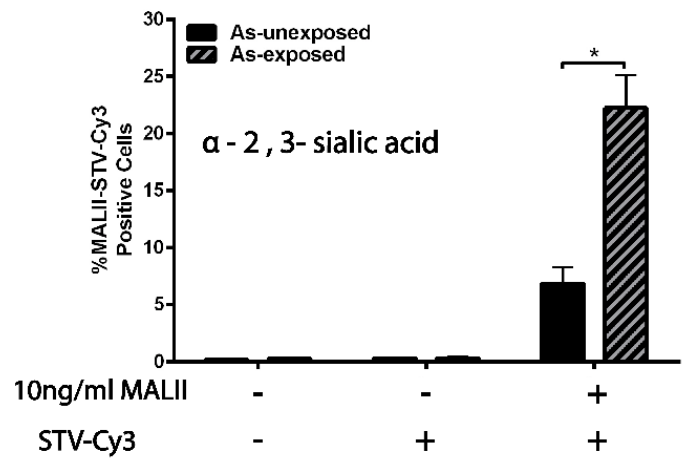

B

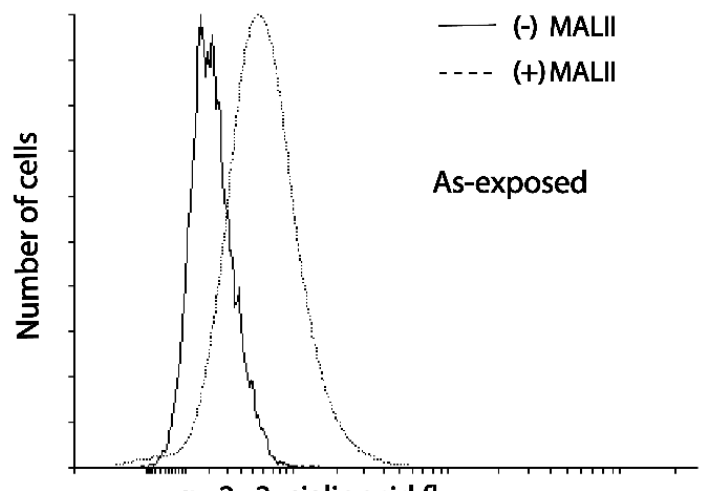

a-2, 3- sialic acid fluorescence

D

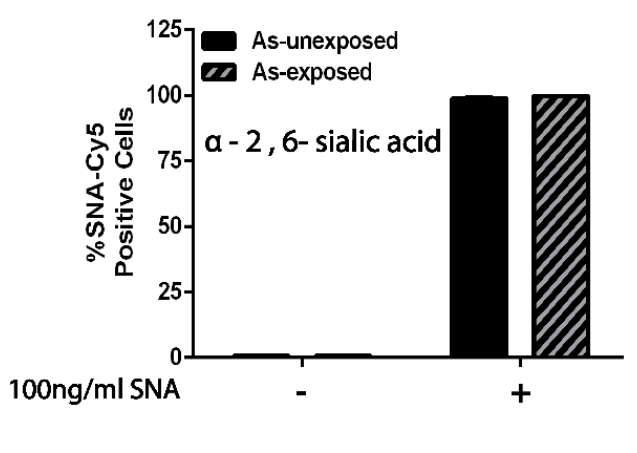

A. A representative cytometric histogram showing $\alpha$-2,3-linked sialic acid fluorescence signal (x-axis) in As-unexposed MDCK cells (solid line represents MALII negative population and dashed line represents MALII positive population). B. A representative cytometric histogram showing $\alpha-2,3$-linked sialic acid fluorescence signal (x-axis) in Asexposed MDCK cells (solid line represents MALII negative population and dashed line represents MALII positive population) C. Percentage of MALII-positive cells in Asunexposed and As-exposed MDCK cells. Error bars represent means \pm SEM. $n=3$ independent biological replicates. $*=p<0.05$. D. Percentage of SNA-positive cells in Asunexposed and As-exposed MDCK cells. Error bars represent means \pm SEM. $n=3$ independent biological replicates. 


\section{CHAPTER 3: CHRONIC ARSENITE EXPOSURE ENHANCES INFLUENZA VIRUS INFECTION IN HUMAN LUNG EPITHELIAL CELL LINES}

Some text and figures in this section were adapted from: Amouzougan EA, Lira, R, \& Klimecki, WT. (2019) Journal of Applied Toxicology.

\subsection{ABSTRACT}

Humans are continuously exposed to harmful toxicants in the environment. Arsenic is a naturally occurring toxicant that is widely distributed in the environment. Sources of arsenic in the environment come from natural and anthropogenic activities. Humans are primarily exposed to arsenic through drinking water, air, and food. In humans, exposure to arsenic has been associated with malignant and non-malignant diseases. There is emerging evidence suggesting that arsenic exposure may worsen disease outcomes from respiratory infection in humans and animals. We have previously demonstrated that chronic arsenite exposure resulted in enhanced virus infection in Madin-Darby canine kidney (MDCK) epithelial cells. Hence, we hypothesized that chronic arsenite exposure could similarly increase influenza virus infection, in human bronchial epithelial (BEAS-2B) and human lung adenocarcinomic alveolar basal (A549) cell lines. In this study, we characterized influenza A/Udorn/72 (H3N2) virus infection in BEAS-2B and A549 cells. We observed a 4.4-fold increase in viral mRNA levels (48h p.i) in chronic arsenite-exposed BEAS-2B cells. Furthermore, arsenite exposure did not significantly change the viral matrix (M2) protein expression in BEAS-2B cells. Additionally, exposure to sodium arsenite did not 
significantly change the viral matrix (M2) protein expression and the viral mRNA quantity in A549 cells at 48 hours post-infection (p.i.). Furthermore, arsenite exposure did not significantly alter virus attachment and the expression of $\alpha-2,3-$ linked sialic acid in BEAS2B cells. However, arsenite exposure significantly reduced cell viability and increased cytotoxicity in virus infected BEAS-2B and A549 cells. This suggests that exposure to arsenic can increase influenza virus infection in human lung epithelial cell lines.

\subsection{INTRODUCTION}

It has been reported that exposure to arsenic targets multiple organ systems, including the respiratory system (Mohammed Abdul, Jayasinghe, Chandana, Jayasumana, \& De Silva, 2015). Numerous epidemiological studies have linked arsenic exposure through drinking water to increased morbidity due to respiratory diseases in humans. In a population-based study, Parvez and colleagues evaluated the effects of arsenic exposure on lung function. The authors found that low to moderate doses of arsenic exposure is associated with significant lung impairment and deleterious non-malignant lung effects (Parvez et al., 2013). The effects of arsenic exposure on pulmonary function was also investigated by Mazumder and colleagues in West Bengal, India. A cross-sectional study that revealed long term-exposure to inorganic arsenic, negatively impacted pulmonary function leading to adverse respiratory effects (Guha Mazumder \& Dasgupta, 2011). Laboratory studies have also shown that exposure to arsenic may have deleterious effect on lung function and worsen respiratory disease outcomes in animals (Ramsey, Foong, et al., 2013). In utero arsenic exposure has also been found to increase the risk of respiratory tract infection in infant mice through perturbation of lung development and modulating the expression of 
genes involved in innate immunity (Ramsey, Bosco, et al., 2013). Furthermore, arsenic exposure has also been demonstrated to alter the immune response to influenza virus infection and subsequently increase the mortality of mice (Kozul, Ely, et al., 2009). Influenza viruses cause severe respiratory diseases in humans and animals. Annual epidemic and occasional pandemic outbreaks of influenza virus have been associated with significant morbidity and mortality worldwide. Influenza remains one of the main public health concerns due to its continuous threat to global health (Taubenberger \& Morens, 2008). It has been estimated that 3-5 million people are infected with influenza virus each year, resulting in 250,000 - 500,000 deaths each year (Who, 2011). Laboratory studies suggest that arsenic exposure can exacerbate influenza virus infections and may lead to increased mortality due to infection.

These emerging studies have focused on the effect of arsenic exposure on the immune system resulting in increased morbidity caused by influenza infection. Lung epithelial cells serve as a physical barrier against infection and play an important role in the innate immune defense. They produce anti-viral factors such as cytokines to activate both the adaptive and innate immune responses (Vareille et al., 2011a). Previous studies have shown that arsenic exposure can disrupt proper tight junction formation, cause the loss of epithelial cells identity, and induce malignant transformation in human airway epithelial cells (Sherwood, Liguori, et al., 2013; Zhao et al., 2014; Zhong et al., 2018). Damage to the airway epithelium can lead to increase bacterial and virus infections (Berkes, Viswanathan, Savkovic, \& Hecht, 2003; Luo et al., 2017). Despite the numerous reports in the literature about the impact of arsenic exposure on the modulation of biological processes in epithelial 
cell models, the influence of respiratory viruses on arsenic-exposed epithelial cells remain unknown. We have recently demonstrated that chronic exposure to sodium arsenite enhances influenza virus infection in MDCK cells. Based on our previous findings we have hypothesized that chronic exposure to arsenic can alter airway epithelial cell biology, leading to enhanced influenza virus infection in vitro.

In this present study, we characterized influenza A/Udorn/72 virus infection in human lung epithelial cells. This study may provide insights into novel molecular mechanisms by which arsenic enhances influenza virus infection in vitro.

\subsection{METHOD}

\section{Cell Culture and Arsenite Exposure}

Human bronchial epithelial cells (BEAS-2B) were purchased from American Type Collection (ATCC, Manassas, VA) and grown in bronchial epithelial cell growth medium (BEGM; Lonza, Walkersville, MD) with supplements (BEGM BulletKit; Lonza). Human alveolar basal epithelial cells (A549) were also purchased from ATCC (Manassas, VA) and grown in Dubelcco's Modified Eagle Medium (DMEM: Gibco, Grand Island, NY) media supplemented with 10\% FBS (Gibco) and 100 units/mL of Antibiotic-Antimycotic (ABAM; Fisher Scientific Waltham, MA). BEAS-2B and A549 cells were cultured in 75 $\mathrm{cm}^{2}$ Falcon tissue culture treated flasks (ThermoFisher Scientific). BEAS-2B and A549 cells were plated in $75 \mathrm{~cm}^{2}$ flask at a density of 500,000 cells/flask and culture media was replaced every 2 days. All cell lines were passaged every 3 days at 80-90\% confluence and were maintained in a humidified incubator at $37{ }^{\circ} \mathrm{C}$ and $5 \% \mathrm{CO}_{2}$ atmosphere. Arsenite- 
exposed cells were continuously treated with $1 \mu \mathrm{M}$ of sodium arsenite in media and were passage-matched to arsenite-exposed cells.

\section{Virus Propagation}

The influenza A virus strain, A/udorn/1972 (H3N2) was kindly provided by Dr. Jun Wang at the University of Arizona. The virus was propagated in MDCK cells cultured in DMEM (DMEM: Gibco, Grand Island, NY) containing 0.5\% bovine serum albumin (BSA: Sigma Aldrich, St. Louis, MO) and $2 \mu \mathrm{g} / \mathrm{mL} \mathrm{N-acetyl} \mathrm{trypsin} \mathrm{(Sigma} \mathrm{Aldrich,} \mathrm{St.} \mathrm{Louis,} \mathrm{MO).}$ Cell culture supernatant was harvested 48 hours post-infection and centrifuged at $300 \mathrm{rpm}$ for 30 minutes to remove cell debris. Virus titers were determined by utilizing a plaque assay with MDCK cells.

\section{Plaque Assay}

MDCK cells were seeded in 6-well plates and grown to 90-100\% confluence then infected with $1 \times 10^{4} \mathrm{pfu}$ of the influenza A virus strain, A/udorn/1972 (H3N2) virus in DMEM media containing $0.5 \% \mathrm{BSA}$. The plate was incubated at $4{ }^{\circ} \mathrm{C}$ for one hour to allow virus attachment and then a one hour incubation at $37{ }^{\circ} \mathrm{C}$ to allow virus internalization. After the incubation, cells were washed with PBS and covered with 1x DMEM media containing 1.2\% Avicel and $2 \mu \mathrm{g} / \mathrm{mL} \mathrm{N}$-acetyl trypsin (Sigma Aldrich, St. Louis, MO). Cells were further incubated for 48 hours at $37^{\circ} \mathrm{C}$ and $5 \% \mathrm{CO}_{2}$ atmosphere to allow plaque formation. Plaque images were captured using a tripod-mounted Nikon D3200 DSLR with a shutter speed of $1 / 30$ second with the $(50 \mathrm{~mm})$ lens aperture set to $\mathrm{f} / 16$. Images were processed and analyzed using ImageJ version 1.50i (NIH, Bethesda, MD). A macro was set up to 
automate image processing/analysis commands and a single set of parameters were used to process images. Images were converted to a binary image by applying an otshu threshold. Images were then inverted to set the background to white and the pixel size was set to 250-infinity.

\section{Immunoblotting}

Arsenite-unexposed and arsenite-exposed cells were seeded in 6-well plates at the density of 500,000 cells/well. Cells were infected with influenza A/udorn/1972 (H3N2) at the concentration of $1 \times 10^{9} \mathrm{pfu}$ (plaque forming units) 48 hours after seeding. Cells were further incubated for additional 48 hours at $37{ }^{\circ} \mathrm{C}$ and $5 \% \mathrm{CO}_{2}$ for 48 hours. Cells were washed twice with cold PBS and lysed in NP40 Cells Lysis Buffer [(pH 7.4), $150 \mathrm{mM} \mathrm{NaCl}, 50$ mM EDTA, $50 \mathrm{mM}$ Tris, and 1\% Nonidet P40 (NP40)] supplemented with 1x MS-SAFE Protease and Phosphatase Inhibitor (MilliporeSigma, Darmstadt, Germany). Cells were incubated on ice for 20 minutes and sonicated after the incubation period. Samples were centrifuged at $12,000 \mathrm{rpm}$ for 20 minutes at $4{ }^{\circ} \mathrm{C}$ to remove cell debris. Pierce $660 \mathrm{~nm}$ Protein Assay Reagent (ThermoFisher Scientific, Waltham, MA) was used to determine total protein concentration. Sodium dodecyl sulfate polyacrylamide gel electrophoresis (SDS-PAGE) technique was used to separate proteins, and proteins were then blotted on nitrocellulose membrane (Bio-Rad, Hercules, CA). Anti-M2 (GeneTex, Irvine, CA) and anti-GAPDH (Santa Cruz Biotechnology, Dallas, TX), were used as primary antibodies. Primary antibodies were used at a concentration of 1:5000 for anti-GAPDH and 1:1000 for anti-M2. All secondary antibodies were purchased from purchased from Abcam (Cambridge, United Kingdom) and were used at a concentration of 1:5000. Immunoblots 
were visualized using ECL Western Blotting Substrate (ThermoFisher Scientific) and detected using a ChemiDoc XRS+ imaging system (Bio-Rad, Hercules, CA).

\section{Quantitative Real-Time PCR (qRT-PCR)}

Arsenite-unexposed and arsenite-exposed cells were seeded in 6-well plates at the density of 500,000 cells/well. Cells were infected with influenza A/udorn/1972 (H3N2) at the concentration of $1 \times 10^{9} \mathrm{pfu}$ (plaque forming units) 48 hours after seeding. RNeasy Mini Kit (Qiagen, Valencia, CA) was used to extract total RNA from cells, and RNA concentration was determined using NanoDrop 2000 spectrophotometer (ThermoFisher Scientific, Waltham, MA). TaqMan RNA-to-CT-1-Step Kit (ThermoFisher Scientific, Waltham, MA) was used to perform quantitative real-time PCR using StepOnePlus Real Time PCR system (Life Technologies; Grand Island, NY) according to the manufacturer instructions. Each reaction contained 20 ng of total cellular RNA. Human RPLPO (Large Ribosomal Protein) copy number was measured in each cDNA as an internal control with the following TaqMan assay IDs: RPLPO (Hs99999902_m1), which was purchased from Thermofisher Scientific (Waltham, MA). Previously published primer and probe sequences (Centres et al., 2017; Yoshida et al., 2011) were used to measure influenza A matrix gene mRNA levels. The published primers and probe used to target influenza matrix gene were custommade by Thermofisher Scientific (Waltham, MA) and the sequences were as follows: MP39-67 Forward: 5'-CCMAGGTCGAAACGTAYGTTCTCTCTATC-3', MP-183-153 Reverse: 5'-TGACAGRATYGGTCTTGTCTTTAGCCAYTCCA-3', and MP-96-75 ProbeAs 5'-(FAM)-ATYTCGGCTTTGAGGGGGCCTC- (MGB)-3'. The qRT-PCR results were analyzed using $2^{-\Delta \Delta_{C}}$ method previously described by Schmittgen et al. 
(Livak \& Schmittgen, 2001) using StepOne Software v2.3 (Applied Biosystems; Waltham, MA).

\section{Cell Viability Assay}

Arsenite-unexposed and arsenite-exposed cells were seeded into clear 96-well microplates at the concentration of 4,000 cells/well in growth medium. Twenty-four hours after seeding, cells were treated with various concentrations $\left(0-4.0 \times 10^{7} \mathrm{pfu}\right)$ of influenza A/udorn/72 (H3N2). The plate was incubated in a humidified incubator at $37{ }^{\circ} \mathrm{C}$ and $5 \%$ $\mathrm{CO}_{2}$ atmosphere for 72 hours. CellTiter 96 Aqueous One Solution Cell Proliferation Assay (MTS, Promega, Madison, WI) was used to measure cell viability according to manufacturer guidelines. After 72 hours post-infection, each well was treated with $20 \mu \mathrm{l}$ of MTS/PMS solution, and the plate was further incubated for 1.5 hours in a humidified incubator at $37{ }^{\circ} \mathrm{C}$ temperature and $5 \% \mathrm{CO}_{2}$ atmosphere. Synergy HTX Multi-Mode Microplate Reader (BioTek, Winooski, VT) was used to measure the conversion of tetrazolium to formazan at the optical absorbance of $490 \mathrm{~nm}$.

\section{Flow Cytometric Analysis: Virus Attachment Assay}

Arsenite-unexposed and arsenite-exposed cells were washed twice with PBS $(100,000$ cells/tube) and were infected with influenza A/udorn/1972 (H3N2) virus (9x109 pfu) at 4 ${ }^{\circ} \mathrm{C}$ for 2 hours to allow virus attachment. After 2 hours, the virus was removed, cells were washed with cold PBS, resuspended in a blocking solution [(10\% BSA and $1 \%$ sodium azide)], and placed on ice for 30 minutes. After the 30 minute incubation period, the blocking solution was removed from cells. Cells were resuspended with influenza A anti- 
NP monoclonal antibody conjugated to fluorescein isothiocyanate (FITC) or isotype control monoclonal antibody conjugated to FITC for 1 hour on ice. Anti-NP and the isotype control antibodies were used at a concentration of 1:10 dilution. All the antibodies were purchased from ThermoFisher Scientific (Waltham, MA). After the incubation period, cells were washed twice with cold PBS and resuspended in 3\% BSA solution. Cells were placed on ice and analyzed by flow cytometry using LSRII flow cytometer (BD Biosciences, San Jose, CA). The percentage of influenza infected positive cells was determined using Flowing Software version 2.5.1 developed by Perttu Terho (Centre for Biotechnology, Finland).

\section{Flow Cytometric Analysis: Sialic Acid Quantification}

To quantify $\alpha-2,6$ sialic acid, arsenite-unexposed and arsenite-exposed (500,000 cells/tube) were washed twice with PBS, and stained with $100 \mathrm{ng} / \mathrm{mL}$ of Sambucus Nigra (SNA) lectin conjugated to cyanine-5 (Cy5) for 30 minutes on ice. After incubation on ice, cells were washed twice with PBS, resuspended in PBS and placed on ice until the flow cytometric analysis of the samples. To quantify $\alpha-2,3$ sialic acid, arsenite-unexposed and arseniteexposed (500,000 cells/tube) were washed twice with PBS, and stained with $10 \mathrm{ng} / \mathrm{mL}$ biotinylated Maackia Amurensis lectin II (MALII) for 30 minutes on ice. After incubation on ice, cells were washed twice with PBS and stained with streptavidin (STV) conjugated to cyanin-3 (Cy3) (Thermofisher Scientific, Waltham, MA) at a concentration of 1:200 dilution to allow for the detection of biotinylated MALII. Cells were washed with PBS, resuspend in PBS and placed on ice until the flow cytometric analysis of the samples. Sambucus Nigra (SNA) lectin conjugated to cyanine-5 (Cy5) and biotinylated Maackia 
Amurensis lectin II (MALII) were purchased from Vector Laboratories (Burlingame, CA). Cells were analyzed by using a LSRII flow cytometer (BD Biosciences, San Jose, CA). The percent of SNA-Cy5 and MALII-Cy3 was determine using Flowing Software version 2.5.1 developed by Perttu Terho (Centre for Biotechnology, Finland).

\subsection{RESULTS}

Sodium Arsenite results in increased plaque formation in arsenite-exposed MDCK cells after infection with $A / U d o r n / 1972$

Plaque assays were used to determine whether chronic exposure to arsenite enhances influenza A/udorn/1972 (H3N2) infection in MDCK cells after 48 hours post-infection with $1 \times 10^{4} \mathrm{pfu}$ of the virus. Arsenite exposure resulted in a $43 \%$ increase in total plaque

number (Figure 12A, 12B) in the arsenite exposed MDCK cells compared to identically infected, arsenite-unexposed MDCK cells. Taken together, these results showed that exposure to arsenite increases influenza A/udorn/1972 (H3N2) virus lytic infection in arsenite-exposed MDCK cells.

Arsenite exposure increased influenza A/Udorn/72 matrix (M) gene expression in BEAS$2 B$ and $A 549$ cells.

After establishing that chronic exposure to $1 \mu \mathrm{M}$ arsenite enhances $\mathrm{A} / \mathrm{Udorn} / 72$ plaque formation in MDCK cells, we evaluated the effects of arsenite exposure on lung relevant human epithelial cell lines (BEAS-2B and A549) in response to influenza A/Udorn/72 infection. We observed a 4.4-fold increase in influenza A matrix $(M)$ gene mRNA levels in arsenite-exposed BEAS-2B cells compared to arsenite-unexposed BEAS-2B cells after 
48 hours post-infection A/Udorn/72 (H3N2). Furthermore, exposure to sodium arsenite did not significantly change the viral mRNA levels A549 cells at 48 hours post-infection (p.i.). These results demonstrate that chronic low doses of arsenite exposure resulted in increased viral mRNA levels in influenza-infected BEAS-2B. Additionally, these results suggest that chronic arsenite exposure did not significantly alter viral mRNA quantity in virus infected A549 cells.

Arsenite exposure increased influenza A/Udorn/ virus Matrix-2 (M2) protein expression BEAS-2B and A549 cells.

We investigated the effects of arsenite exposure on influenza A/Udorn/72 viral protein synthesis in BEAS-2B and A549 cells after 48 hours post-infection. Exposure to sodium arsenite did not significantly change the viral matrix (M2) protein expression in BEAS-2B and A549 cells at 48 hours post-infection (p.i.). These results suggest that chronic arsenite exposure did not significantly alter viral mRNA quantity in virus infected BEAS-2B and A549 cells.

Arsenite exposure increases influenza A/Udorn/72 (H3N2) virus infection in A549

Cell viability assay was used to measure A549 cells response to influenza A/Udorn/72 (H3N2) infection in arsenite-unexposed and arsenite-exposed A549 cells. At seventy-two hours post-infection with the virus, cell viability in arsenite-unexposed and arseniteexposed A549 cells were decreased in a dose-dependent manner. However, we observed more cytotoxicity in virus infected arsenite-exposed A549 cells compared to identically infected, arsenite-unexposed A549 cells. These results showed that exposure to sodium 
arsenite increased cytotoxicity by influenza A/Udorn/72 (H3N2) virus in arsenite-exposed A549 cells.

Arsenite exposure increases influenza A/Udorn/72 (H3N2) virus infection in BEAS-2B

Cell viability assay was used to measure BEAS-2B cells response to influenza A/Udorn/72 (H3N2) infection in arsenite-unexposed and arsenite-exposed BEAS-2B cells. At seventytwo hours post-infection with the virus, cell viability in arsenite-unexposed and arseniteexposed BEAS-2B cells were decreased in a dose-dependent manner. However, we observed more cytotoxicity in virus infected arsenite-exposed BEAS-2B cells compared to identically infected, arsenite-unexposed BEAS-2B cells. These results showed that exposure to sodium arsenite increased cytotoxicity by influenza A/Udorn/72 (H3N2) virus in arsenite-exposed BEAS-2B cells.

Sodium arsenite exposure did not alter influenza A/Udorn/72 (H3N2) virus attachment in infected BEAS-2B cells

We used flow cytometry approach to determine if chronic arsenite exposure impacted virus attachment. We did not observe a significant change in virus attachment in the arseniteexposed BEAS-2B cells compared to arsenite-unexposed BEAS-2B cells (Figure 15). These results suggest that arsenite exposure does not significantly alter influenza virus attachment in BEAS-2B cells. 
Sodium arsenite exposure did not alter $\alpha$-2,3-linked and $\alpha$-2,6-linked sialic acid quantity in BEAS-2B cells.

To determine the effect of arsenic exposure on cell-surface target molecules of influenza binding, we used a flow cytometric approach to quantify $\alpha-2,3$-linked and $\alpha-2,6-$ linked sialic acids in control and arsenite-exposed BEAS-2B cells. Exposure to sodium arsenite did not significantly alter the quantity of $\alpha-2,3$-linked and $\alpha-2,6$-linked sialic acids in this BEAS-2B cells. These results suggest that arsenite exposure does not significantly change the quantity of $\alpha-2,3$-linked and $\alpha-2,6$-linked sialic acids in BEAS-2B cells.

\subsection{DISCUSSION}

We have previously demonstrated that long term exposure to arenite enhanced influenza A/WSN/33 virus infection in MDCK cells. Although MDCK cells are a gold-standard in vitro model for influenza virus research, there are some limitations associated with this model. First, the MDCK cells are derived from kidney epithelial cells. Secondly, the MDCK cell line is not a lung relevant human epithelial cell line. Despites these limitations, the findings from our previous study provided useful insight into some of the potential molecular mechanisms mediating arsenic-enhanced influenza virus infection in vitro. In this present study, we investigated the effects of chronic arsenite exposure on influenza A/Udorn/72 virus infection in immortalized human bronchial epithelial cells (BEAS-2B) and human lung adenocarcinoma cells (A549). 
To our knowledge, this is the first study evaluating the impact of arsenic exposure on influenza A virus infection in human lung epithelial cell lines. BEAS-2B cells were derived from normal bronchial epithelium of non-cancerous individuals and were immortalized using an Adenovirus 12-SV40 hybrid virus or SV40 virus (Ohnuki, Ke, Gerwin, Lechner, \& Harris, 1993; Reddel et al., 1988). The BEAS-2B cell line has been used intensively as an appropriate in vitro model to study lung function (Reddel et al., 1988; Stewart, Torr, Mohd Jamili, Bosquillon, \& Sayers, 2012; Zhao \& Klimecki, 2015). A549 cells were derived from human alveolar basal epithelia cells of a cancerous individual. They have been used in numerous studies as an in vitro model to study lung cancer (Cooper et al., 2016; Gazdar, Girard, Lockwood, Lam, \& Minna, 2010).

The primary step of influenza virus infection is the attachment of the virus to the host cell. This process is mediated by influenza virus receptor known as $\mathrm{N}$-acetylneuraminic acid (sialic acid). Influenza virus binds $\alpha-2,6$-linked and $\alpha$-2,3-linked sialic acids on the cell surface and enter host cell via endocytosis (García-Sastre, 2010). Studies revealed that human lung epithelial cells express both the $\alpha$-2,6-linked and $\alpha$-2,3-linked sialic acids receptors, making them appropriate models for influenza virus research (Fukushima et al., 2014; Matrosovich, Matrosovich, Gray, Roberts, \& Klenk, 2004). Thus, BEAS-2B and A549 cells were used as models for arsenic-exposed human epithelial cell lines in this study.

We began by characterizing influenza A/Udorn/72 (H3N2) virus infection in arseniteexposed MDCK cells using the plaque assay to measure virus infectivity. Arsenite 
exposure resulted in increased plaque formation in $\mathrm{A} / \mathrm{Udorn} / 72(\mathrm{H} 3 \mathrm{~N} 2)$ virus infected cells, revealing that arsenite exposure enhanced virus infection in MDCK cells (Figure 12). This result is consistent with our previous report showing that arsenic exposure resulted in increased plaque formation after influenza $\mathrm{A} / \mathrm{WSN} / 33$ virus infection in MDCK cells.

To show that arsenic exposure could increase influenza A/Udorn/72 virus infection in BEAS-2B and A549 cells, we measured virus mRNA and protein synthesis in virus infected cells. Our results showed that chronic arsenite exposure resulted in increased viral gene expression in arsenite-exposed BEAS-2B. Furthermore, arsenite exposure did not significantly alter viral gene expression in arsenite-exposed A549 cells. We also demonstrated that exposure to arsenite did not significantly alter viral protein expression in arsenite-exposed BEAS-2B and A549 cells. Viral mRNA and protein synthesis are key steps of virus replication (Dou et al., 2018; Matsuoka et al., 2013). Increases in virus protein and gene expression could have functional consequences in influenza infected cells. Therefore, we measured cell viability in arsenite-exposed BEAS-2B and A549 cells after infection with the influenza virus. Our data revealed that influenza $\mathrm{A} / \mathrm{Udorn} / 72$ virus infection resulted in significantly decreased cell viability and increased cytotoxicity in arsenite-exposed BEAS-2B and A549 cells relative to arsenite-unexposed BEAS-2B and A549 cells. Taken together, these results revealed that arsenite exposure resulted in enhanced influenza A/Udorn/72 virus infection in both BEAS-2B and A549 cells. This led us to investigate the potential molecular mechanisms underlying the enhanced influenza virus infection in these human epithelial cell lines. 
Influenza virus replication involves multiples steps, which are attachment, entry, uncoating, replication, assembly, and budding (Dou et al., 2018; Matsuoka et al., 2013). To identify the steps in the influenza virus replication cycle that are affected by arsenic exposure, we investigated the effects of arsenic exposure on virus attachment by performing flow cytometric analysis. Our data showed that chronic arsenic exposure did not significantly change influenza virus attachment in BEAS-2B cells (Figure 15).

As mentioned above, $\alpha-2-3-$ linked and $\alpha-2-6-$ linked sialic acids are the main influenza virus receptors (García-Sastre, 2010; T Ito et al., 1997; Mair, Ludwig, Herrmann, \& Sieben, 2014). To relatively quantify the amount of $\alpha-2-3-$ linked and $\alpha-2-6$-linked sialic acids in arsenite-unexposed and arsenite-exposed BEAS-2B cells, we used microbial lectins that bind sialic acid with specific linkage. Chronic arsenite exposure did not change the quantity of $\alpha-2,3-$ linked and $\alpha-2,6$-linked sialic acids in BEAS-2B cells (Figure 16 and 17).

In this study, we have confirmed previous reports that arsenic exposure increases susceptibility to influenza virus infection in vitro. In our previous report, we showed that arsenite exposure enhances influenza A/WSN/33 virus infection in MDCK cells. The molecular mechanisms were attributed to an increase in $\alpha-2-3$-linked sialic acid expression, and an increase in virus attachment, resulting in enhanced virus infection in arseniteexposed MDCK cells. On the contrary, the molecular mechanisms by which arsenicenhanced influenza virus infection in BEAS-2B cells were not mediated by the alterations of $\alpha$-2-3-linked sialic acid expression and virus attachment. 
In the present study we demonstrated that chronic arsenite exposure enhances influenza A/Udorn/72 virus infection in BEAS-2B and A549 cells. The importance of this study is that arsenite exposure enhances influenza virus infection in human relevant lung epithelial cells. Furthermore, the data suggests that arsenic-enhanced virus infection is generalizable to various strains of influenza virus and different cell line models. However, the molecular mechanisms by which arsenic-enhanced influenza virus infection in BEAS-2B cells is not fully understood. More research is needed to discern all the potential mechanism mediating arsenic-enhanced influenza virus infection. 


\section{Conclusions}

1. Chronic exposure of BEAS2B and A549 cells to sodium arsenite (1 $\mu \mathrm{M})$ at non-toxic levels did not change influenza viral protein levels and mRNA quantity in virus-exposed cells.

2. Sodium arsenite exposure of BEAS-2B and A549 decreases cell viability in arsenite-exposed BEAS-2B and arsenite-exposed A549 cells after infection with influenza virus.

3. Chronic exposure of BEAS2B cells to sodium arsenite did not significantly change virus attachment in virus exposed cells.

4. Sodium arsenite exposure did not significantly alter $\alpha 2,3$-linked sialic acid quantity in arsenite-exposed BEAS-2B cells.

5. Sodium arsenite exposure did not significantly change $\alpha 2,6$-linked sialic acid quantity arsenite-exposed BEAS-2B. 
Figure 12 : Sodium arsenite exposure leads to increased plaque formation in MDCK cells at $48 \mathrm{~h}$ p.i with influenza $\mathrm{A} / \mathrm{WSN} / 33$ (H1N1) virus.

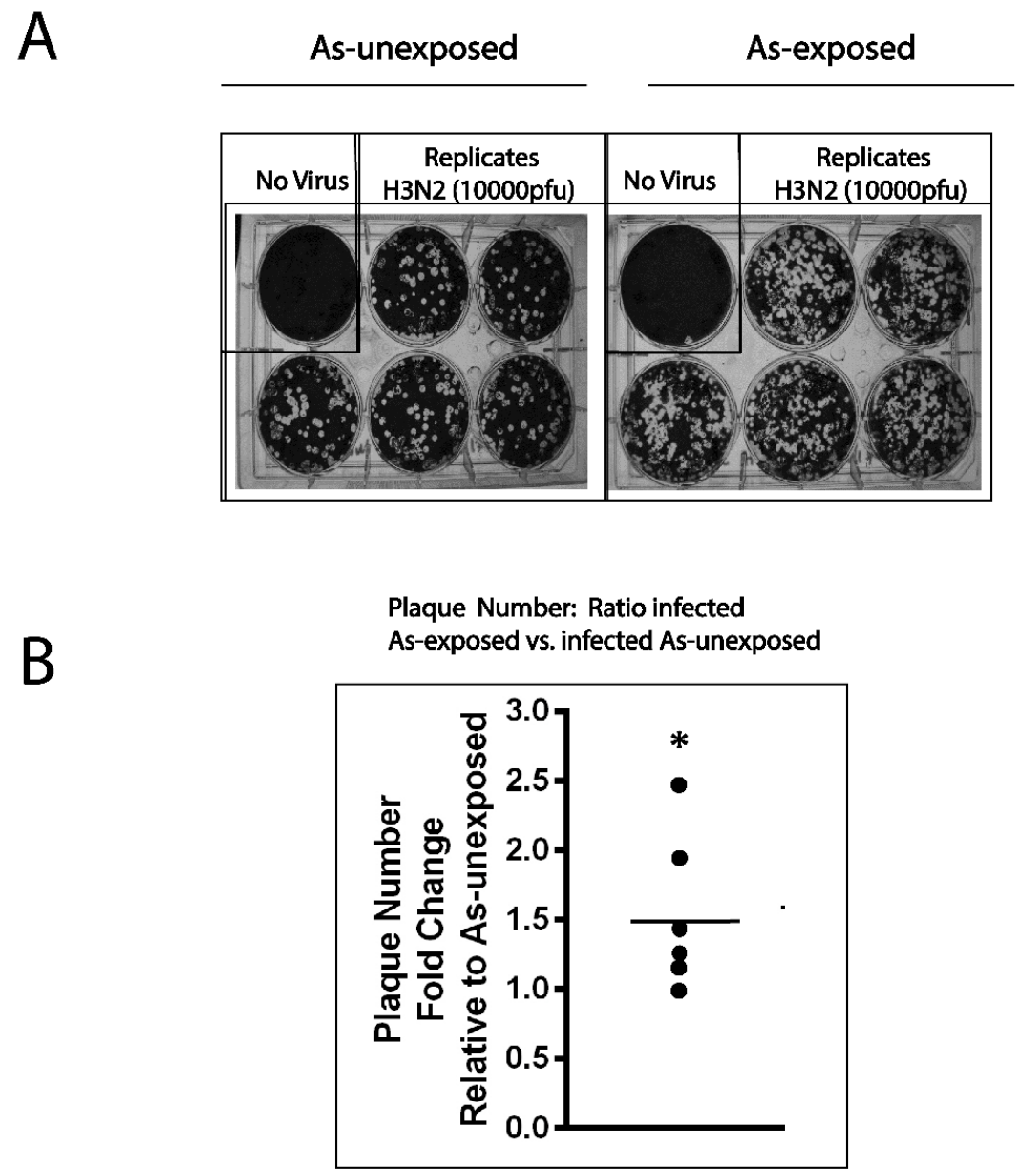

A. Plaque produced in As-unexposed and As-exposed MDCK cells at $48 \mathrm{~h}$ p.i with influenza A/Udorn/72 (H3N2) virus. B. Total plaque number in As-unexposed and Asexposed MDCK cells at 48h p.i with influenza A/Udorn/72 (H3N2) virus. Error bars represent means \pm SEM. $n=5$ (technical replicates), data from 6 independent biological replicates. One sample t-test was used to determine statistical significance between influenza A/Udorn/72 (H3N2) infected As-unexposed and As-exposed MDCK cells. *=p $<0.05$. 
Figure 13: Exposure to sodium arsenite enhanced influenza A/Udorn/72 (H3N2) virus infection in BEAS-2B cells.

A

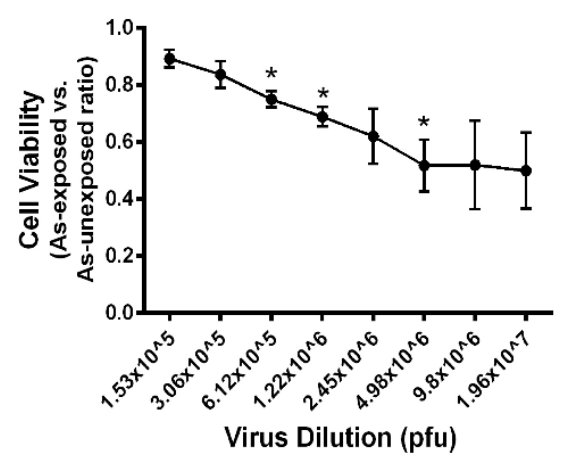

C

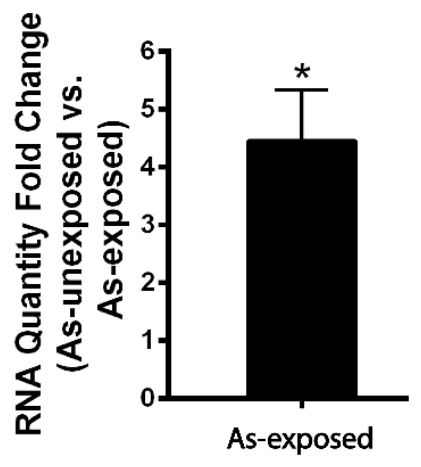

B

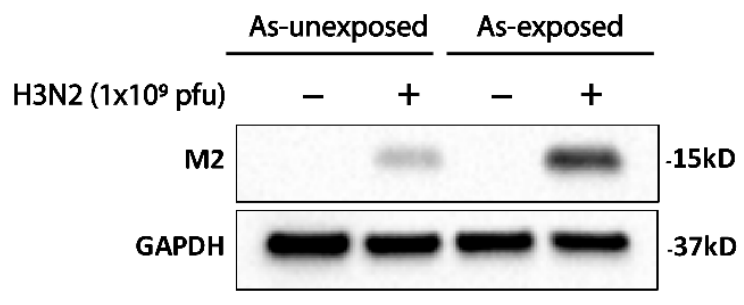

D

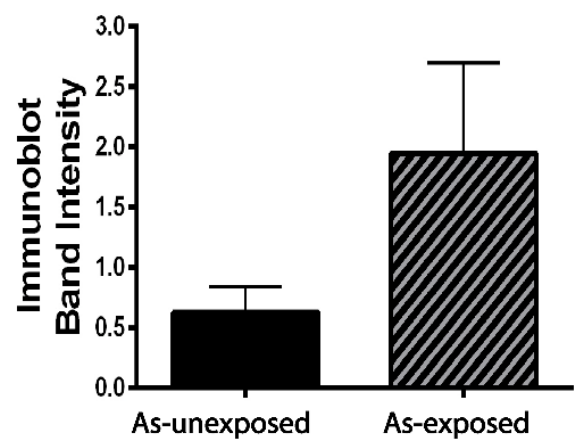

A. Cell viability in As-unexposed and As-exposed BEAS-2B cells treated with $\left(1.5 \times 10^{5}\right.$ $2.0 \times 10^{7} \mathrm{pfu}$ ) of influenza A/Udorn/72 (H3N2) for $72 \mathrm{~h}$. Error bars represent mean \pm standard error of the mean (SEM). $\mathrm{n}=6$ (technical replicates), data from 3 independent biological replicates. $*=p<0.05$. B. Representative immunoblots showing viral matrix (M2) protein in influenza A virus infected As-unexposed and As-exposed BEAS-2B cells at $48 \mathrm{~h}$ p.i. C. Viral matrix gene (M) quantity in influenza A virus infected As-unexposed and As-exposed BEAS-2B cells at $48 \mathrm{~h}$ p.i. Error bar represents mean \pm SEM. $n=4$ independent biological replicates (normalized to arsenite-unexposed BEAS-2B cells fold change $=1$ ). D. Immunoblot band intensity of M2 normalized to GAPDH. Error bars represent mean \pm SEM. $n=3$ independent biological replicates. ${ }^{*}=p<0.05$. 
Figure 14: Exposure to sodium arsenite enhanced influenza A/Udorn/72 (H3N2) virus infection in $\mathbf{A 5 4 9}$ cells.

A

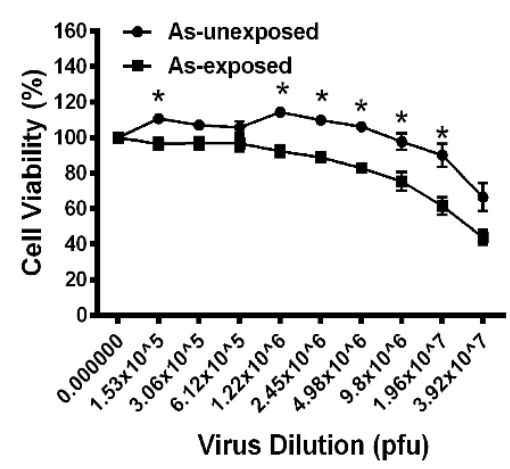

C

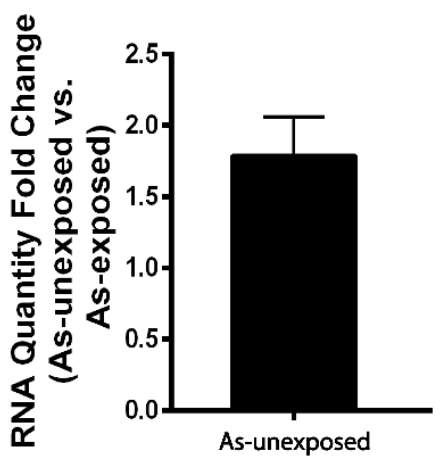

B

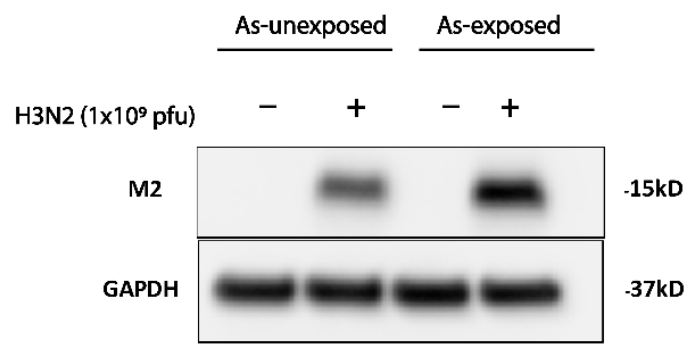

D

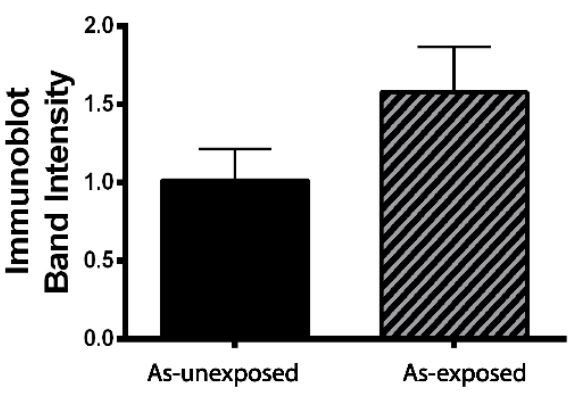

A. Cell viability in As-unexposed and As-exposed A549 cells treated with $\left(0-4.0 \times 10^{7}\right.$ $\mathrm{pfu})$ of influenza A/Udorn/72 (H3N2) for 72h. Error bars represent mean \pm standard error of the mean (SEM). $\mathrm{n}=6$ (technical replicates), data from 3 independent biological replicates. $*=p<0.05$. B. Representative immunoblots showing viral matrix (M2) protein in influenza A virus infected As-unexposed and As-exposed A549 cells at 48h p.i. C. Viral matrix gene (M) quantity in influenza A virus infected As-unexposed and Asexposed A549 cells at $48 \mathrm{~h}$ p.i. Error bar represents mean \pm SEM. $\mathrm{n}=3$ independent biological replicates (normalized to arsenite-unexposed A549 cells fold change $=1$ ). $\mathbf{D}$. Immunoblot band intensity of M2 normalized to GAPDH. Error bars represent mean \pm SEM. $\mathrm{n}=3$ independent biological replicates. 
Figure 15: Exposure to sodium arsenite enhanced influenza A/Udorn/72 (H3N2) virus attachment in BEAS-2B cells.

A

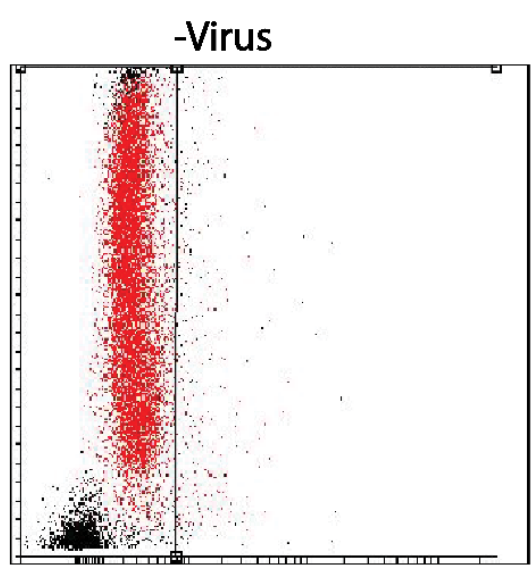

H3N2 nucleoprotein fluorescence
B

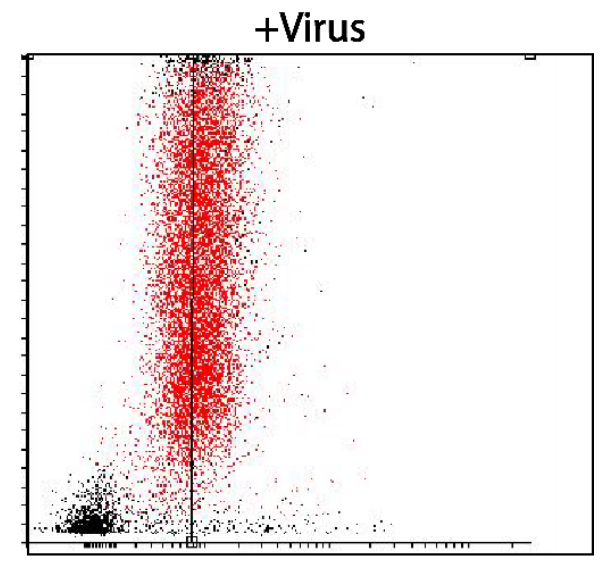

H3N2 nucleoprotein fluorescence

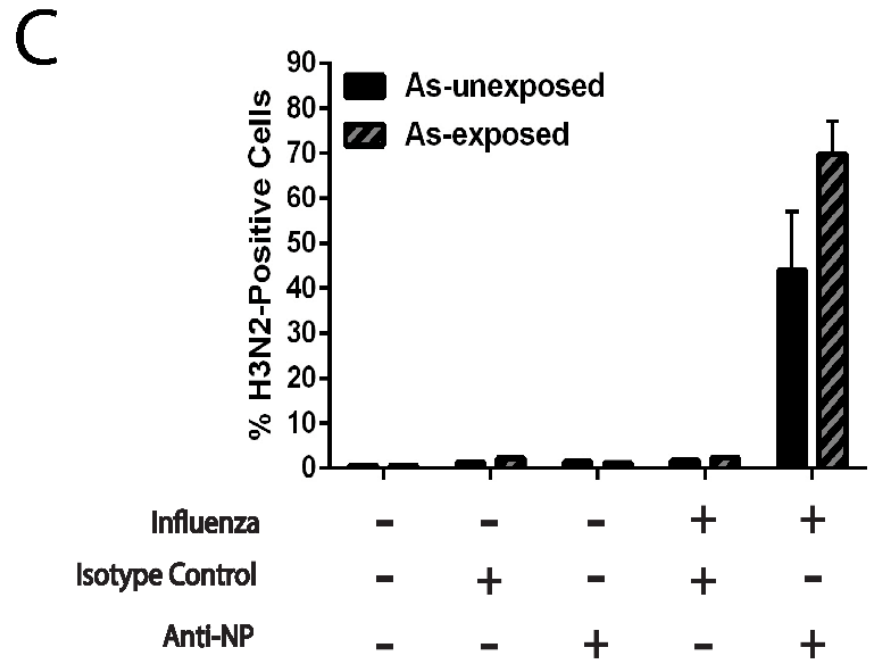

A. A representative cytometric dot plot showing H3N2 nucleoprotein fluorescence signal (x-axis) in uninfected BEAS-2B cells. B. A representative cytometric dot plot showing H3N2 nucleoprotein fluorescence signal ( $\mathrm{x}$-axis) in virus infected BEAS-2B cells C. Percentage of H3N2-positive cells in As-unexposed and Asexposed BEAS-2B cells at $2 \mathrm{~h}$ p.i. Error bars represent means \pm SEM. $\mathrm{n}=3$ independent biological replicates. 
Figure 16: Exposure to sodium arsenite increases $\alpha-2,3$-linked sialic acid quantity in BEAS-2B cells

A

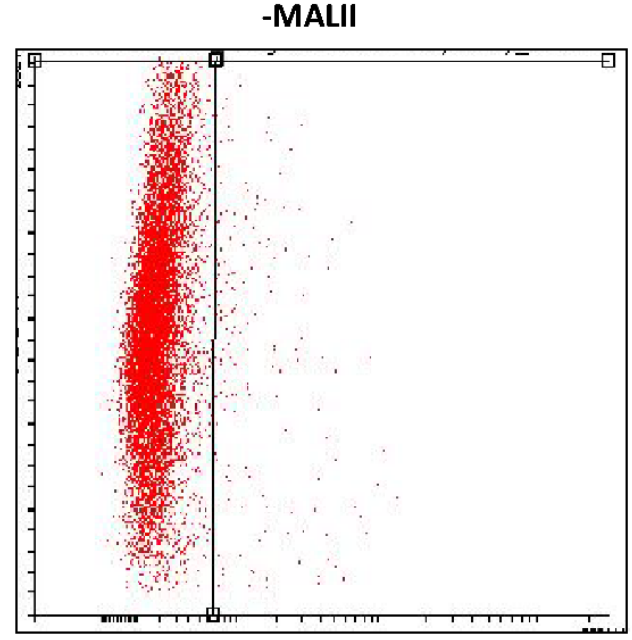

$a-2,3-$ sialic acid fluorescence

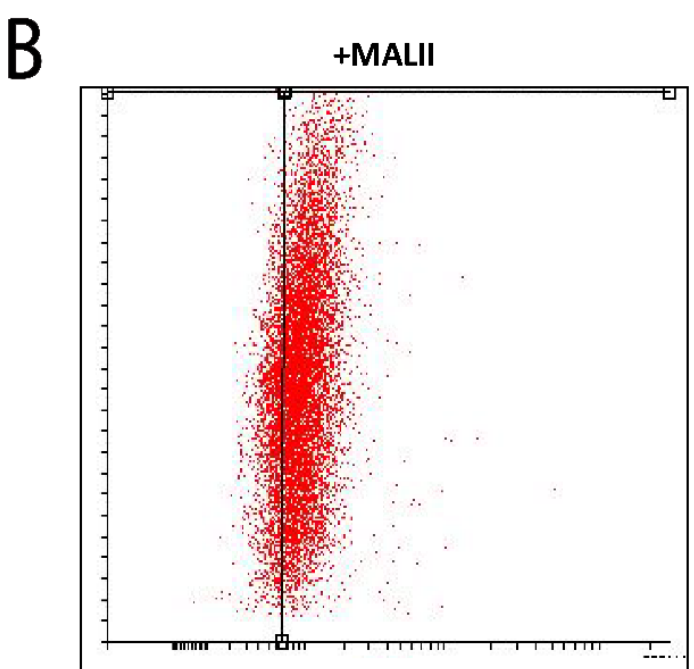

$a-2,3-$ sialic acid fluorescence

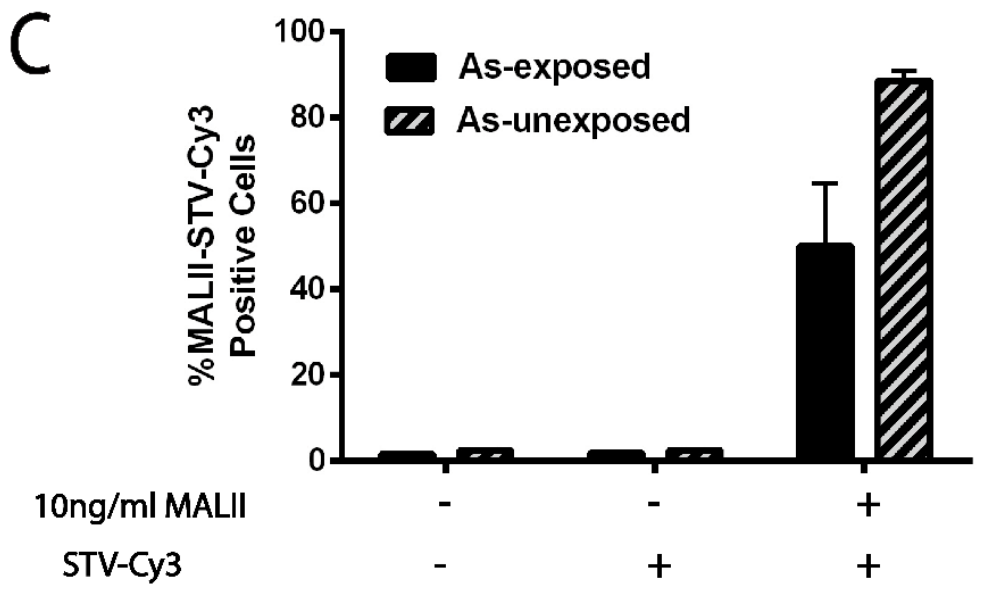

A. A representative cytometric dot plot showing $\alpha$-2,3-linked sialic acid fluorescence signal (x-axis) in MALII negative BEAS-2B cells B. A representative cytometric dot plot showing $\alpha$-2,3-linked sialic acid fluorescence signal ( $\mathrm{x}$-axis) in MALII positive BEAS2B cells C. Percentage of MALII-positive cells in As-unexposed and As-exposed BEAS$2 B$ cells. Error bars represent means \pm SEM. $n=3$ independent biological replicates. 
Figure 17: $\alpha-2,6$-linked sialic acid quantity in BEAS-2B cells

A

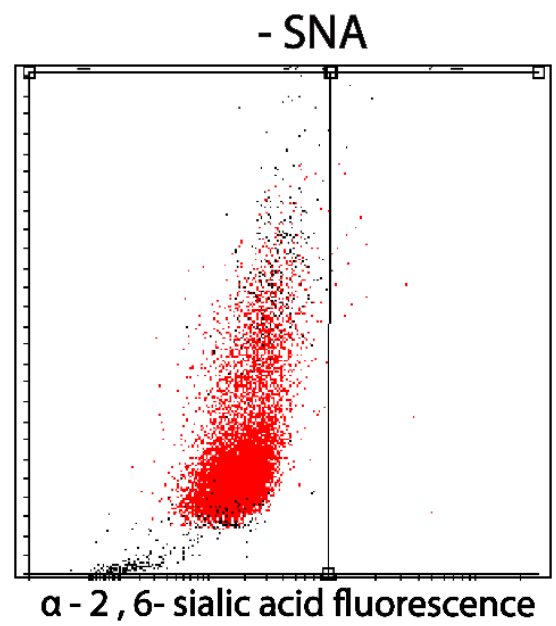

B

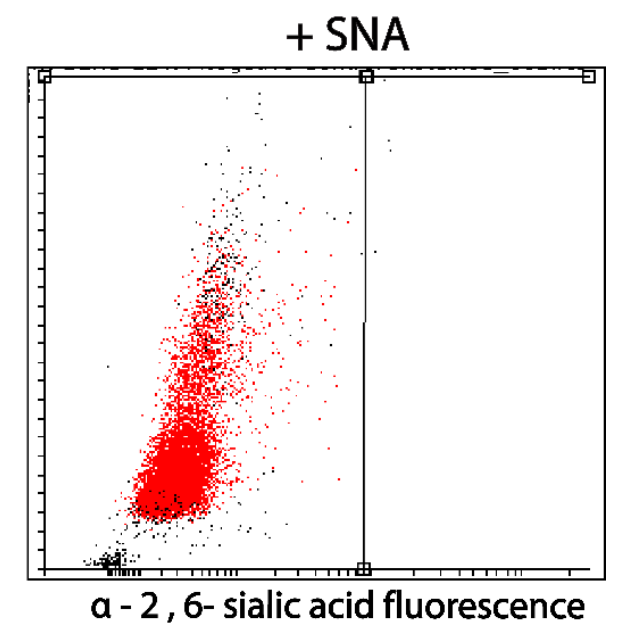

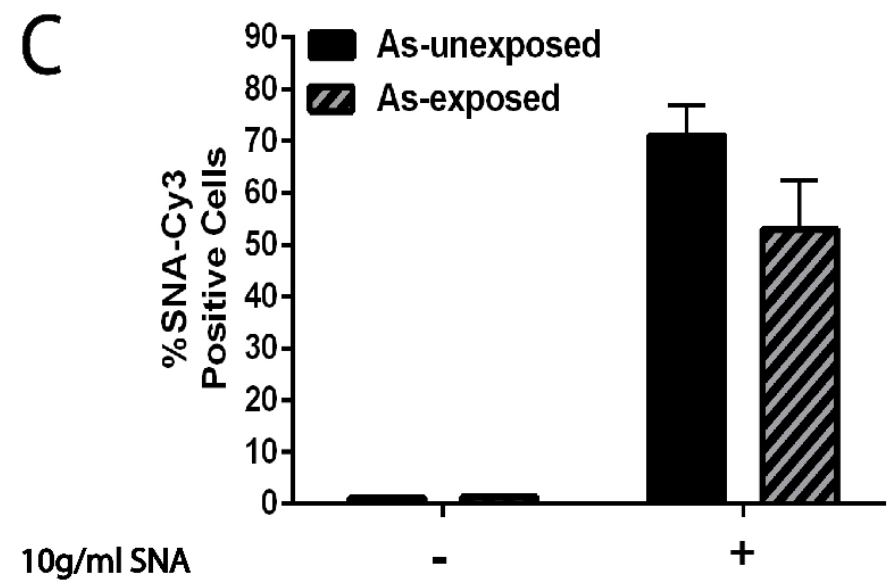

A. A representative cytometric dot plot showing $\alpha$-2,6-linked sialic acid fluorescence signal (x-axis) in SNA negative BEAS-2B cells. B. A representative cytometric dot plot showing $\alpha-2,6$-linked sialic acid fluorescence signal ( $\mathrm{x}$-axis) in SNA positive BEAS-2B cells C. Percentage of SNA-positive cells in As-unexposed and As-exposed BEAS-2B cells. Error bars represent means \pm SEM. $n=3$ independent biological replicates. 


\section{APPENDIX A: ARSENITE EXPOSURE MEDIATES INHIBITION OF PI3K/AKT SIGNALING PATHWAY IN INFLUENZA INFECTED CELLS}

The work presented in this chapter was not fully completed. However, this chapter contained valuable findings that provided insights into the molecular mechanisms mediating arsenite-enhanced influenza virus infection in vitro.

\subsection{ABSTRACT}

The phosphatidylinositol-3-kinase (PI3K)/Akt pathway has been shown to play an important role in influenza virus infection. The activation of Akt signaling pathway has been associated with enhanced influenza virus infection. Several studies show that influenza virus NS1 protein activates the PI3K/Akt signaling pathway to increase virus propagation. Here we showed that exposure to sodium arsenite inhibits the PI3K/Akt activation in influenza $\mathrm{A} / \mathrm{WSN} / 33$ virus infected MDCK cells. However, inhibition of the PI3K/Akt activity affected virus replication in the arsenite-exposed MDCK cells. Interestingly, we observed enhanced influenza virus infection in the arsenite-exposed MDCK cells. We observed a 3.0-fold decreased in phospho-Akt protein expression in the arsenite-exposed MDCK cells compared to arsenite-unexposed MDCK cells after infection with influenza virus $(24 \mathrm{~h} \mathrm{p.i).} \mathrm{This} \mathrm{data} \mathrm{suggests} \mathrm{that} \mathrm{arsenite} \mathrm{enhanced} \mathrm{influenza} \mathrm{virus}$ infection in MDCK cells could be through PI3K/Akt-independent mechanisms. 


\subsection{INTRODUCTION}

It has been reported that influenza virus interacts with numerous host cellular signaling pathways for an efficient virus replication (W. Dong et al., 2014; Watanabe, Watanabe, \& Kawaoka, 2010). Specifically, influenza virus has been shown to modulate the phosphatidylinositol-3-kinase (PI3K/Akt) signaling pathway during infection (Ehrhardt \& Ludwig, 2009). The PI3K/Akt pathway plays an important role in many cellular processes such as cell survival, cell cycle progression, cell growth, and apoptosis (Altomare \& Testa, 2005; X. Zhang, Tang, Hadden, \& Rishi, 2011). Studies show that influenza virus nonstructural protein 1 (NS1) can activate the PI3K/Akt signaling pathway to enhance virus replication (Ehrhardt et al., 2006; Li et al., 2012). This led us to investigate the status of the PI3K/Akt signaling pathway in arsenite-exposed MDCK cells after infection with influenza A/WSN/33 (H1N1) virus.

PI3K/Akt is activated through its phosphorylation at Ser473 and Thr308 by mTORC2 (mammalian target of rapamycin complex 2) and PDK1 (3'-phosphoinositide-dependent kinase 1) (Kuss-Duerkop et al., 2017; Palmieri et al., 2017). Once activated, Akt can trigger downstream activation of many substrates (Dozza, Smith, Perry, Tabaton, \& Strocchi, 2004; Fang et al., 2000; Guo, Snider, \& Chen, 2016). Interestingly, we observed low phospho-Akt protein expression in arsenite-exposed virus-infected MDCK cells relative to virus-infected control MDCK cells. This suggests that inactivation of the PI3K/Akt signaling pathway due to arsenite exposure did not inhibit influenza virus replication. 


\subsection{METHOD}

\section{Immunoblotting}

Control and arsenite-exposed cells were seeded at a density of 500,000 cells/well in 6-well plates. Forty-eight hours after seeding, cells were infected with influenza A/WSN/33 (H1N1) virus (1005 pfu) in $\mathrm{N}$-acetyl trypsin $(2 \mu \mathrm{g} / \mathrm{ml})$ containing media. The plate was further incubated at $37^{\circ} \mathrm{C}$ and $5 \% \mathrm{CO}_{2}$ atmosphere for an additional 24 hours. After incubation, cells were washed twice with cold PBS and lysed in NP40 Cells Lysis Buffer [(pH 7.4), 150mM NaCl, 50mM EDTA, 50mM Tris, and 1\% Nonidet P40 (NP40)]. Cell lysis buffer was supplemented with 1x MS-SAFE Protease and Phosphatase Inhibitor (MilliporeSigma, Darmstadt, Germany). Samples were sonicated and subjected to centrifugation at $12,000 \mathrm{rpm}$ for 20 minutes at $4^{\circ} \mathrm{C}$ to remove cell debris. After sonication, total protein in the supernatant was quantified using the Pierce $660 \mathrm{~nm}$ Protein Assay Reagent (ThermoFisher Scientific, Waltham, MA). Samples were subject to sodium dodecyl sulfate polyacrylamide gel electrophoresis (SDS-PAGE), and separated proteins were blotted on nitrocellulose membrane (Bio-Rad, Hercules, CA). Anti-GAPDH (Santa Cruz Biotechnology, Dallas, TX), Anti-Akt, and Anti-phoshoAkt (Cell Signaling Technology, Danvers, MA) antibodies were used for protein detection. All horseradish peroxidase-secondary antibodies were purchased from Santa Cruz Biotechnology (Dallas, TX). Anti-GAPDH, was used at a concentration of 1:5000. Anti-Akt, and Anti-phoshoAkt were used at a concentration 1:1000. Secondary antibodies were used at concentration of 1:5000. Anti-GAPDH antibody was used as loading control for all western blots. Immunoblots were visualized using ECL Western Blotting Substrate (ThermoFisher 
Scientific) and detected using a ChemiDoc XRS+ imaging system (Bio-Rad, Hercules, CA).

\subsection{RESULTS}

Influenza A/WSN/33 (H1N1) virus infection decreases p-Akt levels in arsenite-exposed cells

We investigated the impact of arsenite exposure on the PI3K/Akt signaling pathway in virus infected MDCK cells (24h p.i). Immunoblot analysis revealed significantly decreased (3.0-fold) levels of phospho-Akt protein in the arsenite-exposed MDCK cells relative to arsenite-unexposed MDCK cells (24 p.i) (Figure, 18). There were no significant differences observed in total Akt protein levels in arsenite-unexposed and arsenite-exposed MDCK cells. Taken together, our data suggests that influenza A/WSN/33 (H1N1) virus infection negatively impacts Akt activation in arsenite-exposed MDCK compared to unexposed MDCK.

\subsection{DISCUSSION}

In this study we showed that influenza A virus infection failed to activate the PI3K/Akt signaling pathway following chronic exposure to sodium arsenite. We observed a significant decrease (3-fold) in phospho-Akt protein expression in the arsenite-exposed virus-infected MDCK cells (24 h p.i.) (Figure 18). Akt is activated in a biphasic manner during infection. It has been reported that Akt activation is transient during the early stage of infection, followed by a sustained activation in the late stage of infection (Ehrhardt et al., 2006). We speculate that upon infection with influenza A/WSN/33 (H1N1) virus, Akt 
is activated similarly in both the arsenite-exposed and arsenite-unexposed MDCK cells to promote virus entry. However, in the late stage of infection, arsenic mediates virus-induced loss of Akt kinase activity. This suggests that in the arsenite-exposed MDCK cells, influenza virus does not require Akt activity for enhanced virus replication. We observed enhanced virus infection in the arsenite-exposed MDCK cells, suggesting that arsenite enhanced influenza virus infection is through mechanisms that are independent of the (PI3K)/Akt signaling pathway. However, more research is needed to understand the mechanisms behind arsenite-mediated inactivation of PI3K/Akt signaling pathway in influenza infected cells and its impact on virus replication.

There are some limitations that are associated with this study. First, overexpression of phospho-Akt is needed to determine whether that could reverse the enhanced virus infection observed in the arsenite-exposed MDCK cells. Second, siRNA knockdown of phospho-Akt protein expression is needed to determine if complete loss of phospho-Akt correlates with more enhanced infection in the arsenite-exposed MDCK cells. Lastly, future studies are needed to confirm these results. Nonetheless, these results provide insights into novel potential mechanisms mediating arsenite-enhanced influenza infection in vitro.

\section{Conclusion}

1. Chronic exposure of MDCK cells to sodium arsenite $(1 \mu \mathrm{M})$ correlates with decreased phospho-Akt expression. 
Figure 18 : Exposure to sodium arsenite correlates with decreased phospho-Akt expression in MDCK cells

A

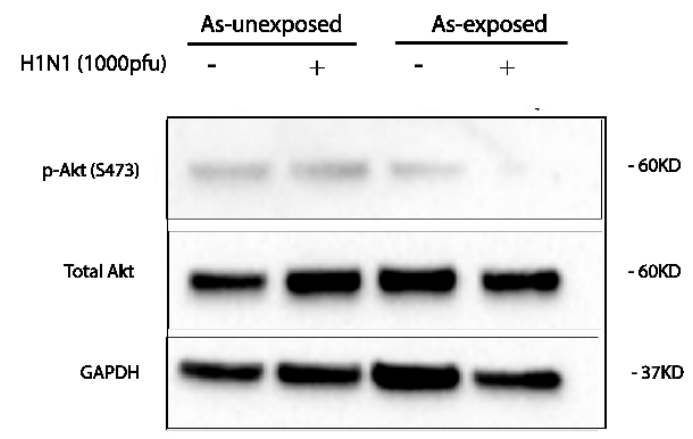

B

C
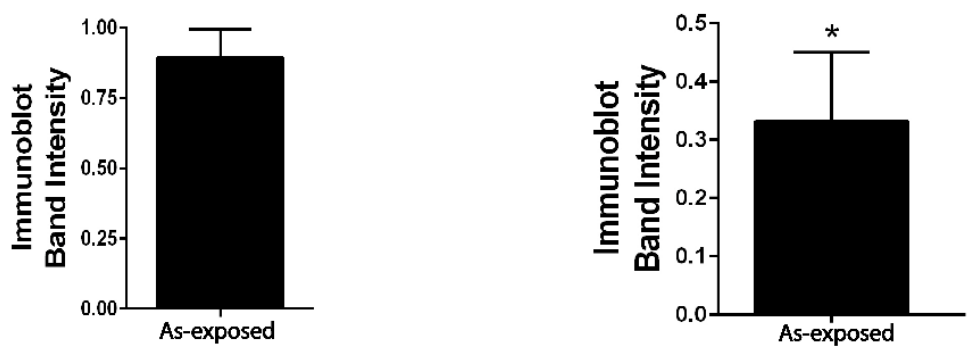

A. A representative immunoblots showing total Akt and phospo-Akt protein levels in influenza A virus infected As-unexposed and As-exposed MDCK cells. B. Immunoblots band intensity of total Akt normalized to GAPDH. Error bar represent mean \pm SEM. $n=3$ independent biological replicates. C. Immunoblots band intensity of phosphoAkt normalized to GAPDH. Error bar represent mean \pm SEM. $\mathrm{n}=3$ independent biological replicates. ${ }^{*}=\mathrm{p}<0.05$ 


\section{APPENDIX B: ARSENITE EXPOSURE INHIBITS TRIM22 AND MX1 PROTEINS EXPRESSION IN BEAS-2B CELLS}

The work presented in this chapter was not fully completed. However, this chapter contained valuable findings that provided insights into the molecular mechanisms mediating arseniteenhanced influenza virus infection in vitro.

\subsection{ABSTRACT}

Interferons (IFNs) are family of cytokines that play important roles in the innate immune defense due to their ability to induce the expression of antiviral genes. Specifically, interferons induce interferon-stimulated genes (ISGs) that can directly inhibit virus infection and replication. TBK1, Mx1 and TRIM22 have been shown to have antiviral activity against influenza viruses. In this study we demonstrated that exposure to sodium arsenite resulted in decreased (1.83-fold) TBK1 protein expression and decreased (2.85-fold) Mx1 protein expression in the arsenite-exposed BEAS-2B cells compared to arsenite-unexposed BEAS-2B cells. Furthermore, Arsenite exposure resulted in decreased (2.00-fold) TRIM22 mRNA quantity in the arsenite-exposed BEAS-2B cells. Thus, this study suggests arsenite-enhanced influenza virus infection in BEAS-2B could be in part due to disruption of the interferon response pathways.

\subsection{INTRODUCTION}

Cytokines play an important role in host cellular defense against infection, and proinflammatory cytokines are generally known to exacerbate various diseases. There are various types of cytokines, 
including proinflammatory cytokines, chemokines, anti-inflammatory cytokines, and anti-viral cytokines (J.-M. Zhang \& Jianxiong, 2009). Interferons (IFNs) are a family of cytokines that possess antiviral activities and are known to directly block virus replication. Interferon alpha (IFN$\alpha)$ and interferon beta (IFN- $\beta$ ) are known as type I interferons. Interferon gamma (INF- $\gamma$ ) and lambda interferon (INF- $\lambda$ ) are known as type II and type III interferon respectively (Ank et al., 2006; A. J. Lee \& Ashkar, 2018). Type I, and type III interferons can be secreted by all cell types, whereas type II interferons are produced by immune cells. During infection a number of viruses bind to pattern recognition receptors (PRRs) such as Toll-like receptors (TLRs), C-type lectin receptors (CLRs), retinoic acid-inducible gene-I-like receptors (RLRs), and nucleotide binding oligomerization domain-(NOD) like receptors (NLRs), leading to the production of interferons and the expression of interferon-stimulated genes (ISGs) (Jang et al., 2015). ISGs play an important role in antiviral defense against pathogens (Schneider, Chevillotte, \& Rice, 2014).

Tank-binding kinase (TBK1) is a serine/threonine kinase that is critical in the transcriptional regulation of hundreds of ISGs. DNA and RNA viruses bind to PRRs, leading to the activation of TBK1. The TBK1 protein phosphorylates interferon regulatory factors (IRFs), resulting in the activation of the antiviral state. Phosphorylation of IRF3 and IRF7 lead to the dimerization and nuclear translocation with interferon-stimulated response element (ISRE), resulting in the production of type I interferons (IFN- $\alpha$ and IFN- $\beta$ ) and the induction of ISGs (Hu et al., 2018).

In vivo and in vitro studies show that arsenic exposure downregulates the expression of type I and type II interferons and the interferon-stimulated genes such as myxovirus resistance $1(\mathrm{Mx} 1)$ protein (Dangleben, Skibola, \& Smith, 2013). Mx1 is transcriptionally induced by type I (IFN- $\alpha$ 
and IFN- $\beta$ ) and type III (IFN- $\lambda$ ) interferons (Verhelst, Parthoens, Schepens, Fiers, \& Saelens, 2012). It belongs to a family of dynamin-like GTPase that are involved in antiviral defense against infection (Haller \& Kochs, 2002). It has also been shown to inhibit influenza virus replication through the interaction with viral ribonucleoprotein (Verhelst et al., 2012). Additionally, Mx1 has been shown to block the replication of highly pathogenic influenza virus strain H5N1 in mice (Salomon et al., 2007). In zebrafish embryos, arsenite exposure has been shown to inhibit the expression of antiviral and antibacterial genes, including $i l 1 b$, tnfa, ifnphi (type 1 interferon), and $m x$ (interferon-inducible $\mathrm{Mx}$ ) gene, leading to suppression of the innate immune function (Nayak et al., 2007). Interferons are also known to induce tripartite motif (TRIM) proteins, which have been shown to inhibit the replication of several viruses. Overexpression of TRIM22 has been shown to block the replication of human immunodeficiency virus 1 (HIV-1), hepatitis B virus (HBV), and encephalomyocarditis virus (ECMV) (Bouazzaoui et al., 2006; Di Pietro et al., 2013). Furthermore, it has also been shown to inhibit influenza A (H1N1) virus replication through the degradation of virus nucleoprotein. Lastly, inhibition of TRIM22 resulted in enhanced virus replication in adenocarcinomic human alveolar basal epithelial (A549) cells (Di Pietro et al., 2013). These studies show that interferons (IFNs) and interferon-stimulated genes (ISGs) play an important role in the innate immune defense against infection. Based on these studies, we hypothesized that arsenic-induced inhibition of interferon-stimulated genes (ISGs) could enhance influenza virus infection in vitro. In this study, we demonstrated that exposure to sodium arsenite resulted in decreased expression of TRIM22 and Mx1 proteins in human bronchial lung epithelial (BEAS-2B) cells. This study may provide insights into novel molecular mechanisms through which arsenite could enhance influenza virus infection in humans. 


\subsection{METHOD}

\section{Immunoblotting}

Control and arsenite-exposed cells were seeded at a density of 500,000 cells/well in 6-well plates. Forty-eight hours after seeding, cells were infected with influenza A/Udorn/72 (H1N1) virus $\left(1 \times 10^{9} \mathrm{pfu}\right)$ in $\mathrm{N}$-acetyl trypsin $(2 \mu \mathrm{g} / \mathrm{ml})$ containing media. The plate was further incubated at $37^{\circ} \mathrm{C}$ and 5\% $\mathrm{CO}_{2}$ atmosphere for an additional 24 hours. After incubation, cells were washed twice with cold PBS and lysed in NP40 Cells Lysis Buffer [(pH 7.4), 150mM NaCl, 50mM EDTA, 50mM Tris, and 1\% Nonidet P40 (NP40)]. Cell lysis buffer was supplemented with 1x MS-SAFE Protease and Phosphatase Inhibitor (MilliporeSigma, Darmstadt, Germany). Samples were sonicated and subjected to centrifugation at $12,000 \mathrm{rpm}$ for 20 minutes at $4^{\circ} \mathrm{C}$ to remove cell debris. After sonication, total protein in the supernatant was quantified using the Pierce $660 \mathrm{~nm}$ Protein Assay Reagent (ThermoFisher Scientific, Waltham, MA). Samples were subject to sodium dodecyl sulfate polyacrylamide gel electrophoresis (SDS-PAGE), and separated proteins were blotted on nitrocellulose membrane (Bio-Rad, Hercules, CA). Anti-GAPDH (Santa Cruz Biotechnology, Dallas, TX), Anti-TBK1, and Anti-Mx1 (Santa Cruz Biotechnology, Dallas, TX) antibodies were used for protein detection. All horseradish peroxidase-secondary antibodies were purchased from Santa Cruz Biotechnology (Dallas, TX). Anti-GAPDH, was used at a concentration of 1:5000. Anti-TBK1, and Anti-Mx1 were used at a concentration 1:1000. Secondary antibodies were used at concentration of 1:5000. Anti-GAPDH antibody was used as loading control for all western blots. Immunoblots were visualized using ECL Western Blotting Substrate (ThermoFisher Scientific) and detected using a ChemiDoc XRS+ imaging system (BioRad, Hercules, CA). 
Quantitative Real-Time PCR ( $q R T-P C R)$

Arsenite-unexposed and arsenite-exposed cells were seeded in 6-well plates at the density of 500,000 cells/well. Cells were infected with influenza A/udorn/1972 (H3N2) at the concentration of $1 \times 10^{9} \mathrm{pfu}$ (plaque forming units) 48 hours after seeding. RNeasy Mini Kit (Qiagen, Valencia, CA) was used to extract the total RNA from cells, and RNA concentration was determined using NanoDrop 2000 spectrophotometer (ThermoFisher Scientific, Waltham, MA). TaqMan RNA-toCT-1-Step Kit (ThermoFisher Scientific, Waltham, MA) was used to perform quantitative realtime PCR using StepOnePlus Real Time PCR system (Life Technologies; Grand Island, NY) according to the manufacturer instructions. Each reaction contained $20 \mathrm{ng}$ of total cellular RNA. Human RPLPO (Large Ribosomal Protein) copy number was measured in each cDNA as an internal control. The following TaqMan assay IDs were used in the assay: RPLPO (Hs99999902_m1), TRIM22 (Hs01001179_ml), and Mx1 (Hs00895608_ml), which were purchased from Thermofisher Scientific (Waltham, MA). The qRT-PCR results were analyzed using $2^{-\Delta \Delta_{C}}$ method previously described by Schmittgen et al. (Livak \& Schmittgen, 2001) using StepOne Software v2.3 (Applied Biosystems; Waltham, MA).

\subsection{RESULTS}

Arsenite exposure decreased the expression of TBK1 and MxI proteins in virus infected BEAS$2 B$ Cells

Immunoblot analysis revealed significantly decreased (1.83-fold) levels of TBK1 protein in the arsenite-exposed BEAS-2B cells relative to arsenite-unexposed BEAS-2B cells at 48 hours postinfection (Figure 19A). Furthermore, we observed a significant decrease (2.85-fold) in Mx1 
protein in the arsenite-exposed BEAS-2B cells (48 h p.i) (Figure 19B). Taken together, these results showed that exposure to sodium arsenite decreased antiviral gene expression in virus infected BEAS-2B cells.

Arsenite exposure decreased MxI and TRIM22 $m R N A$ expression in virus infected BEAS-2B Cells

Mx1 and TRIM22 mRNA quantities were measured using qRT-PCR after infection with A/Udorn/1972 virus. In contrast to Mx1 protein levels, qRT-PCR analysis did not show a significant decrease in Mx1 mRNA quantity in the arsenite-exposed BEAS-2B cells. However, we observed a significant decrease (2.00-fold) in TRIM22 mRNA quantity in the arsenite-exposed BEAS-2B cells compared to arsenite-unexposed BEAS-2B cells.

\subsection{DISCUSSION}

Inhibition of interferon signaling pathways has been shown to be associated with increased susceptibility to bacterial and viral infections (Devasthanam, 2014; Stifter \& Feng, 2015). Exposure to arsenic has been linked to inhibition of interferons (IFNs) and interferon-stimulated genes (ISGs) expression (Hermann \& Kim, 2005; Lage, Nayak, \& Kim, 2006). In this study, we aimed to determine whether arsenite-enhanced influenza virus infection in BEAS-2B cells is in part due to disruption of the interferon signaling pathways. We showed that exposure to sodium arsenite resulted in a significant decrease in TBK1 protein expression in the arsenite-exposed BEAS-2B cells. Inhibition of TBK1 could impaire interferon signaling and the antiviral defense against influenza virus infection in the arsenite-exposed cells. Activation of TBK1 is linked to the expression of antiviral genes, including Mx1 expression. Therefore, we also investigated the effect 
of arsenic exposure on Mx1 protein and mRNA expression in influenza infected BEAS-2B cells. We demonstrated that exposure to sodium arsenite resulted in a significant decrease in Mx1 protein expression in arsenite-exposed BEAS-2B cells relative to arsenite-unexposed BEAS-2B cells. However, arsenite exposure did not significantly change Mx1 mRNA quantity in the arseniteexposed BEAS-2B cells. These results are consistent with previous studies showing that arsenite exposure resulted in decreased Mx1 gene expression in Zebrafish (Nayak et al., 2007).

We also investigated the effect of arsenic exposure on antiviral protein TRIM22. It has been reported that TRIM22 protein is upregulated in response to influenza A virus infections (Di Pietro et al., 2013). We showed that arsenic exposure resulted in a significant decrease in TRIM22 mRNA quantity in the arsenite-exposed BEAS-2B cells compared to arsenite-unexposed BEAS-2B cells. To our knowledge, this is the first study demonstrating that arsenite exposure can decrease the expression of TBK1 and TRIM22 expression in vitro. The reduction in TBK1, Mx1, and TRIM22 expression could impair the antiviral defense mechanisms, leading to enhanced influenza virus infection in the arsenite-exposed BEAS-2B cells. We have previously demonstrated that exposure to arsenic enhanced influenza virus infection in BEAS-2B cells. The molecular mechanisms mediating the enhances influenza virus infection in vitro is not fully understood. These data suggest that arsenic impaired interferon signaling pathways and antiviral response mechanisms may be responsible for the enhanced infection observed in the arsenite-exposed BEAS-2B cells. However, more studies are needed to uncover all the molecular mechanisms mediating arsenicenhanced influenza virus infection in vitro. 
There are some limitations that are associated with this study. First, overexpression of TBK1, Mx1 and TRIM22 are needed to determine whether that could reverse the enhanced virus infection observed in the arsenite-exposed BEAS-2B cells. Second, there are hundreds of interferonstimulated genes and proteins that possess antiviral activity. We investigated the effect of arsenite on several antiviral genes and interferons, including interferon regulatory factor-3 (IRF-3), interferon regulatory factor-7 (IRF-7), interferon-stimulated gene-15 (ISG15), interferon-beta (IFN- $\beta$ ), interferon-gamma (IFN- $\gamma$ ), tank-binding kinase 1 (TBK1), and myoxyvirus resistance 1 (Mx1). However, only TBK1, Mx1, and TRIM22 produced significant results. Therefore, future studies are needed to investigate the effect of arsenite exposure on more genes that are involved in the interferon response pathways. Nonetheless, these results provide novel insights into potential mechanisms mediating arsenic-enhanced influenza virus infection in vitro. 
Figure 19: Exposure to sodium arsenite correlates with decreased TBK1 and Mx1 protein expression in BEAS-2B cells

A
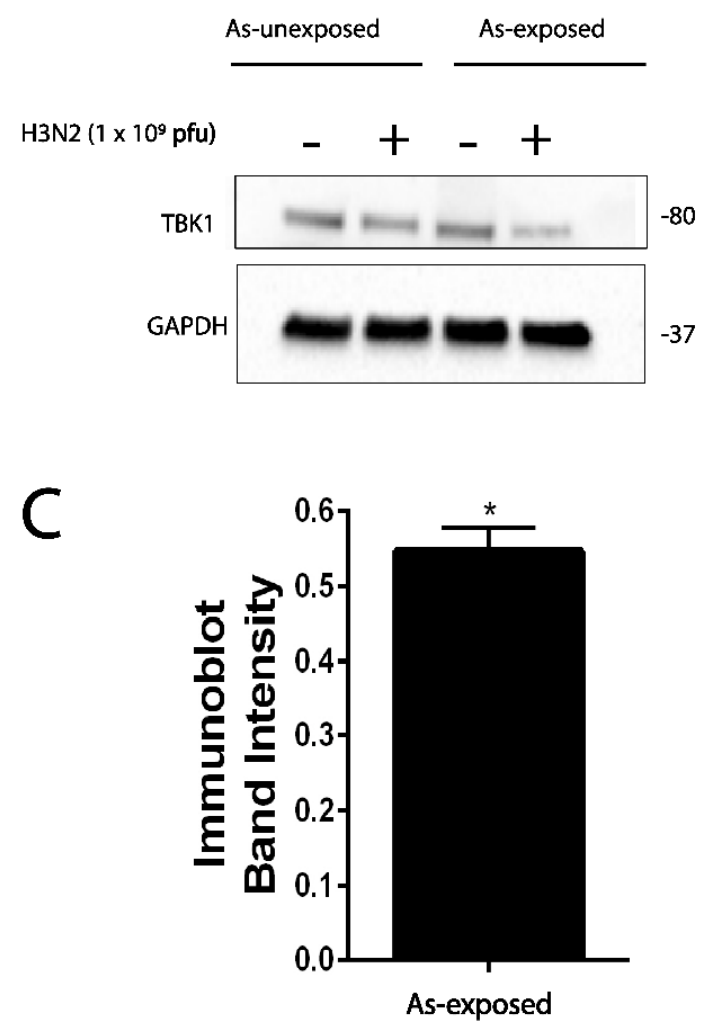

B

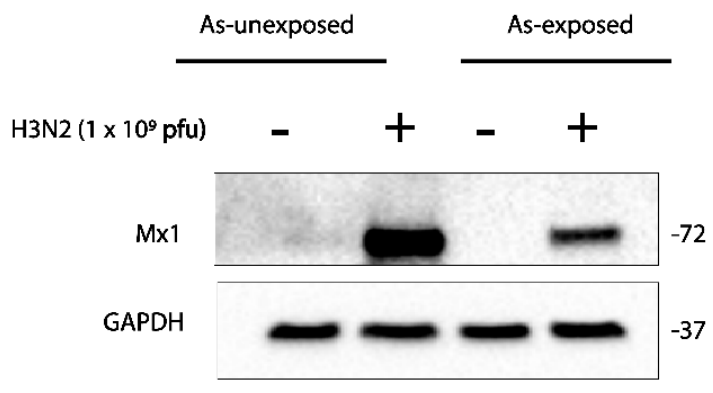

D

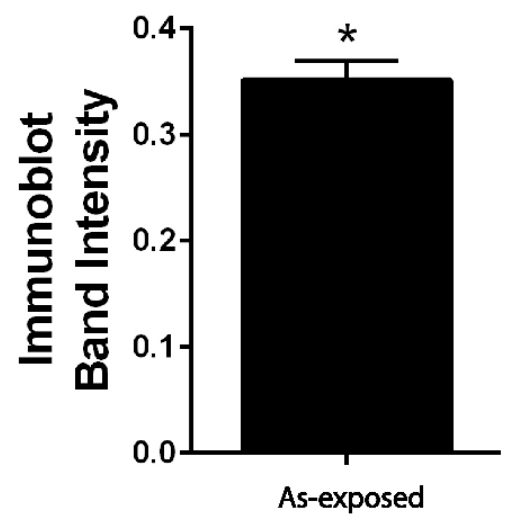

A. A representative immunoblots showing total TBK1 protein levels in influenza A virus infected As-unexposed and As-exposed BEAS-2B cells. B. A representative immunoblots showing total Mx1 protein levels in influenza A virus infected As-unexposed and Asexposed BEAS-2B cells C. Immunoblots band intensity of TBK1 normalized to GAPDH. Error bar represent mean \pm SEM. $\mathrm{n}=3$ independent biological replicates. D. Immunoblots band intensity of Mx1 normalized to GAPDH. Error bar represent mean \pm SEM. $n=3$ independent biological replicates. $*=p<0.05$ 
Figure 20: Exposure to sodium arsenite correlates with decreased TRIM22 mRNA quantity BEAS-2B cells

A
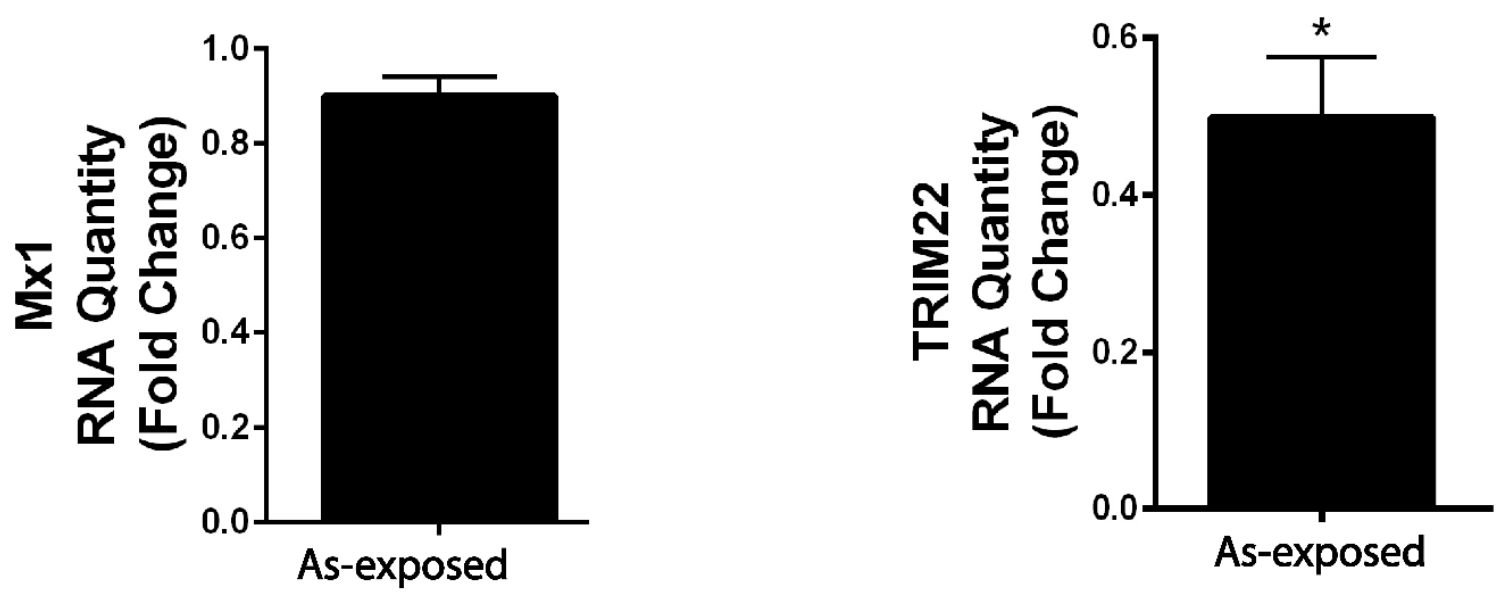

A. Mx1 quantity in influenza A virus infected As-unexposed and As-exposed BEAS-2B cells at $48 \mathrm{~h}$ p.i. Error bars represent means \pm SEM. $\mathrm{n}=3$ independent biological replicates. $\mathbf{B}$. TRIM22 quantity in influenza A virus infected As-unexposed and As-exposed BEAS-2B cells at $48 \mathrm{~h}$ p.i. Error bars represent means \pm SEM. $n=3$ independent biological replicates. Unpaired t-test was used to determine statistical significance between influenza A/Udorn/72 (H3N2) infected As-unexposed and As-exposed A549 cells. $*=p<0.05$. 
Conclusion

1. Chronic exposure of BEAS-2B cells to sodium arsenite $(1 \mu \mathrm{M})$ correlates with decreased Mx1 and TBK1 protein expression.

2. Chronic exposure of BEAS-2B cells to sodium arsenite $(1 \mu \mathrm{M})$ correlates with decreased Mx1 and TRIM22 mRNA quantity. 


\section{FUTURE DIRECTIONS}

The results from these studies provided new insights into novel molecular mechanisms mediating arsenite-enhanced influenza virus infection in vitro. However, these results also raised more questions that should be pursued in the future. Here are some recommendations for the future research directions.

\section{Determine if the machinery following virus attachment is different in control and arsenite- exposed cells post-infection.}

We did not fully assess the impact of arsenite exposure on virus internalization and replication. This can be done using influenza virus expressing red fluorescent protein to directly visualize virus internalization. Using mCherry fluorescent virus could help provide new ways to monitor influenza virus infection in vitro and in vivo. Furthermore, it could also help determine if arseniteenhanced influenza virus infection is mediated through increase virus internalization.

\section{Further characterization of sialic acids role in arsenic-enhanced influenza virus infection in MDCK Cells.}

We demonstrated that arsenite exposure increased $\alpha$-2,3-linked sialic acid expression in MDCK cells. In contrast, arsenite exposure did not change the expression of $\alpha-2,6$-linked sialic acid expression in MDCK cells. However, we did not investigate the molecular mechanisms that lead to the increased expression of $\alpha-2,3$-linked sialic acid and not $\alpha-2,6$-linked sialic acid. This could be done by investigating the effect of arsenic exposure on the key enzymes that are involved in sialic acid biosynthesis such as UDP-N-aetylglucossamine-2-epimerase, $\alpha$-2,3-sialyltransferase, 
$\alpha-2,6$-sialyltransferase, and sialidases. We did not show that siRNA knockdown of these key enzymes could affect the content of $\alpha-2,3$-linked and $\alpha-2,6$-linked sialic acids in MDCK cells. Specifically, we did not show that silencing these enzymes could block the increase in $\alpha$-2,3-linked acid and reverse the enhanced virus infection observed in the arsenite-exposed MDCK cells. This could be done to further determine the role of sialic acids in the enhancement of influenza virus infection.

Further characterization of sialic acids role in arsenic-enhanced influenza virus infection in BEAS-2B Cells.

We have also demonstrated that arsenite exposure enhanced influenza virus infection in lung relevant cell lines (BEAS-2B and A549). We do not fully understand the molecular mechanisms mediating the enhancement of influenza virus infection by arsenite in BEAS-2B and A549 cells. This could be done by investigating the effect of arsenic exposure on the key enzymes that are involved in sialic acid biosynthesis in BEAS-2B cells. Lastly, we did not investigate the effect of arsenite exposure on $\alpha-2,3$-linked and $\alpha-2,6$-linked sialic acids expression in A549 cells. It would be interesting to know how arsenite exposure modulates the expression of sialic acid content in A549 cells.

Create an $\alpha-2,3$ and $\alpha-2,6$ sialyltransferase knockdown mouse model to further determine the role of sialic acids in influenza virus infection in vivo.

It would be interesting to test the effect of arsenite exposure on sialic acid expression in vivo. Previous studies show that arsenic disrupts the innate immune system and increases susceptibility to influenza virus infection in vivo (Kozul, Ely, et al., 2009). Showing that arsenite-enhanced 
influenza virus infection in vivo is also mediated through increased sialic acids expression could be interesting.

Determine the role of arsenite exposure in decreased phospho-Akt protein expression in influenza infected cells.

We have demonstrated that arsenite exposure resulted in loss of phospho-Akt protein expression in the arsenite-exposed infected cells. It could be interesting to determine if loss of phospho-Akt was responsible for arsenite-enhanced influenza virus infection in MDCK cells. This could be done using siRNA to further knockdown phospho-Akt expression or to overexpressed phospho-Akt expression in MDCK cells.

Investigate the effect of arsenite exposure on pathogen recognition receptors. Pathogen recognition receptors (PPRs) such as toll-like receptors (TLRs) play an important role in innate immune defense and induction of the host antiviral genes against infection. It could be interesting to investigate the effect of arsenite exposure on the activation of PPRs, and the interferon signaling pathways.

\section{Characterize influenza virus infection in arsenite-exposed epithelial cells using various strains of influenza virus.}

In our study we demonstrated that arsenite exposure enhanced influenza A/WSN/33 (H1N1) infection in MDCK cells. Furthermore, we showed that exposure to sodium arsenite enhanced influenza A/Udorn/1972 (H3N2) virus infection in BEAS-2B and A549 cells. It would be 
interesting to know if this phenomenon applies to other strains of influenza viruses and other cell types.

\section{Characterize influenza virus infection in arsenite-exposed human primary epithelial cells.}

The use of primary cell model to study arsenic-enhanced influenza virus infection in vitro.

Primary cell lines directly isolated from human or animal tissues could provide more relevant results because they mimic in vivo natural cellular environment. 


\section{REFERENCES}

Air, G. M. (2006). Sequence relationships among the hemagglutinin genes of 12 subtypes of influenza A virus. Proceedings of the National Academy of Sciences, 78(12), 7639-7643. https://doi.org/10.1073/pnas.78.12.7639

Al Hajjar, S., \& McIntosh, K. (2010). The first influenza pandemic of the 21 st century. Annals of Saudi Medicine, 30(1), 1-10. https://doi.org/10.4103/0256-4947.59365

Ali, A., Avalos, R. T., Ponimaskin, E., \& Nayak, D. P. (2002). Influenza Virus Assembly: Effect of Influenza Virus Glycoproteins on the Membrane Association of M1 Protein. Journal of Virology, 74(18), 8709-8719. https://doi.org/10.1128/jvi.74.18.8709-8719.2000

Altomare, D. A., \& Testa, J. R. (2005). Perturbations of the AKT signaling pathway in human cancer. Oncogene, 24(50), 7455-7464. https://doi.org/10.1038/sj.onc.1209085

Anhlan, D., Grundmann, N., Makalowski, W., Ludwig, S., \& Scholtissek, C. (2011). Origin of the 1918 pandemic H1N1 influenza A virus as studied by codon usage patterns and phylogenetic analysis, 64-73. https://doi.org/10.1261/rna.2395211.virus

Ank, N., West, H., Bartholdy, C., Eriksson, K., Thomsen, A. R., \& Paludan, S. R. (2006). Lambda Interferon (IFN- ), a Type III IFN, Is Induced by Viruses and IFNs and Displays Potent Antiviral Activity against Select Virus Infections In Vivo. Journal of Virology, 80(9), 4501-4509. https://doi.org/10.1128/jvi.80.9.4501-4509.2006

Antman, K. H. (2004). Introduction: The History of Arsenic Trioxide in Cancer Therapy. The Oncologist, 6(90002), 1-2. https://doi.org/10.1634/theoncologist.6-suppl_2-1

Asha, K., \& Kumar, B. (2019a). Emerging Influenza D Virus Threat: What We Know so Far! Journal of Clinical Medicine, 8(2), 192. https://doi.org/10.3390/jcm8020192

Asha, K., \& Kumar, B. (2019b). Emerging Influenza D Virus Threat: What We Know so Far! 
Journal of Clinical Medicine, 8(2), 192. https://doi.org/10.3390/jcm8020192

Auewarakul, P., Suptawiwat, O., Kongchanagul, A., Sangma, C., Suzuki, Y., Ungchusak, K., ... Puthavathana, P. (2007). An Avian Influenza H5N1 Virus That Binds to a Human-Type Receptor. Journal of Virology, 81(18), 9950-9955. https://doi.org/10.1128/jvi.00468-07

Ayotte, J. D., Medalie, L., Qi, S. L., Backer, L. C., \& Nolan, B. T. (2017). Estimating the HighArsenic Domestic-Well Population in the Conterminous United States. Environmental Science and Technology, 51(21), 12443-12454. https://doi.org/10.1021/acs.est.7b02881

Aziz, A., Ullah, S. M., \& Ullah, R. (2015). Arsenic in rice grains at Sonargaon, Bangladesh. Bangladesh Journal of Botany, 44(1), 85-89. https://doi.org/10.3329/bjb.v44i1.22728

Baioni, L., Merenda, M., Chiapponi, C., Kyriakis, C. S., Mandola, M. L., Zanni, I., ... Luini, M. V. (2017). Influenza D in Italy: towards a better understanding of an emerging viral infection in swine. Scientific Reports, 7(1), 1-7. https://doi.org/10.1038/s41598-017-120123

Bals, R., \& Hiemstra, P. S. (2004). Innate immunity in the lung: How epithelial cells fight against respiratory pathogens. European Respiratory Journal, 23(2), 327-333. https://doi.org/10.1183/09031936.03.00098803

Barberis, I., Myles, P., Ault, S. K., Bragazzi, N. L., \& Martini, M. (2016). History and evolution of influenza control through vaccination: from the first monovalent vaccine to universal vaccines. Journal of Preventive Medicine and Hygiene, 57(3), E115-E120. Retrieved from http://www.ncbi.nlm.nih.gov/pubmed/27980374\%0Ahttp://www.pubmedcentral.nih.gov/art iclerender.fcgi?artid=PMC5139605

Baris, D., Waddell, R., Beane Freeman, L. E., Schwenn, M., Colt, J. S., Ayotte, J. D., ... Silverman, D. T. (2016). Elevated Bladder Cancer in Northern New England: The Role of 
Drinking Water and Arsenic. Journal of the National Cancer Institute, 108(9), 1-9. https://doi.org/10.1093/jnci/djw099

Berg, M., Zheng, Q., Zhang, Q., Johnson, C. A., Sun, G., Xue, H., \& Rodriguez-Lado, L. (2013). Groundwater Arsenic Contamination Throughout China. Science, 341(6148), 866-868. https://doi.org/10.1126/science. 1237484

Berkes, J., Viswanathan, V. K., Savkovic, S. D., \& Hecht, G. (2003). Intestinal epithelial responses to enteric pathogens: Effects on the tight junction barrier, ion transport, and inflammation. Gut, 52(3), 439-451. https://doi.org/10.1136/gut.52.3.439

Biere, B., Bauer, B., \& Schweiger, B. (2010). Differentiation of influenza b virus lineages yamagata and victoria by real-time PCR. Journal of Clinical Microbiology, 48(4), 14251427. https://doi.org/10.1128/JCM.02116-09

Bodewes, R., Morick, D., de Mutsert, G., Osinga, N., Bestebroer, T., van der Vliet, S., ... Osterhaus, A. D. M. E. (2013). Recurring influenza B virus infections in seals. Emerging Infectious Diseases, 19(3), 511-512. https://doi.org/10.3201/eid1903.120965

Borkenhagen, L. K., Fieldhouse, J. K., Gray, G. C., Zemke, J., Bailey, E. S., Zhang, D., \& Choi, J. Y. (2018). The continual threat of influenza virus infections at the human-animal interface. Evolution, Medicine, and Public Health, 2018(1), 192-198. https://doi.org/10.1093/emph/eoy013

Bos, M. E. H., te Beest, D. E., van Boven, M., van Beest Holle, M. R. R., Meijer, A., Bosman, A., ... Stegeman, A. (2010). High Probability of Avian Influenza Virus (H7N7) Transmission from Poultry to Humans Active in Disease Control on Infected Farms. The Journal of Infectious Diseases, 201(9), 1390-1396. https://doi.org/10.1086/651663

Bouazzaoui, A., Kreutz, M., Eisert, V., Dinauer, N., Heinzelmann, A., Hallenberger, S., ... von 
Briesen, H. (2006). Stimulated trans-acting factor of $50 \mathrm{kDa}$ (Staf50) inhibits HIV-1 replication in human monocyte-derived macrophages. Virology, 356(1-2), 79-94. https://doi.org/10.1016/j.virol.2006.07.025

Bouvier, N. M., \& Palese, P. (2008). The biology of influenza viruses. Vaccine, 26(SUPPL. 4), 49-53. https://doi.org/10.1016/j.vaccine.2008.07.039

Brauer, R., Chen, P., Brauer, R., \& Chen, P. (2016). Influenza leaves a TRAIL to pulmonary edema Find the latest version : Influenza leaves a TRAIL to pulmonary edema, 126(4), 1245-1247. https://doi.org/10.1172/JCI86802.reduce

Bresalier, M. (2012). Uses of a pandemic: Forging the identities of influenza and virus research in interwar Britain. Social History of Medicine, 25(2), 400-424. https://doi.org/10.1093/shm/hkr162

Butt, K. M., Smith, G. J. D., Chen, H., Zhang, L. J., Leung, Y. H. C., Xu, K. M., ... Guan, Y. (2005). Human infection with an avian H9N2 influenza A virus in Hong Kong in 2003. Journal of Clinical Microbiology, 43(11), 5760-5767. https://doi.org/10.1128/JCM.43.11.5760-5767.2005

C., F., \& A.M., S. (2006). Arsenic exposure and its impact on health in Chile. Journal of Health, Population and Nutrition, 24(2), 164-175. https://doi.org/10.2307/23499354

Caini, S., Kroneman, M., Wiegers, T., El Guerche-Séblain, C., \& Paget, J. (2018). Clinical characteristics and severity of influenza infections by virus type, subtype, and lineage: A systematic literature review. Influenza and Other Respiratory Viruses, 12(6), 780-792. https://doi.org/10.1111/irv.12575

Calderón-Garciduenãs, L., Vojdani, A., Blaurock-Busch, E., Busch, Y., Friedle, A., Franco-Lira, M., ... D'Angiulli, A. (2015). Air pollution and children: Neural and tight junction 
antibodies and combustion metals, the role of barrier breakdown and brain immunity in neurodegeneration. Journal of Alzheimer's Disease, 43(3), 1039-1058.

https://doi.org/10.3233/JAD-141365

Centres, W. H. O. C., Cc, W. H. O., July, R., \& Rt-pcr, C. (2017). WHO information for the molecular detection of influenza viruses, (May 2015).

Chakraborti, D., Mahmudur, M., Mukherjee, A., Rahman, M., Morshed, S., Islam, T., \& Sorif, S. (2015). Journal of Trace Elements in Medicine and Biology Groundwater arsenic contamination in Bangladesh -21 Years of research. Journal of Trace Elements in Medicine and Biology, 31, 237-248. https://doi.org/10.1016/j.jtemb.2015.01.003

Checconi, P., Sgarbanti, R., Celestino, I., Limongi, D., Amatore, D., Iuvara, A., ... Nencioni, L. (2013). The environmental pollutant cadmium promotes influenza virus replication in MDCK cells by altering their redox state. International Journal of Molecular Sciences, 14(2), 4148-4162. https://doi.org/10.3390/ijms 14024148

Chen, Y., Graziano, J. H., Parvez, F., Liu, M., Slavkovich, V., Kalra, T., ... Ahsan, H. (2011). Arsenic exposure from drinking water and mortality from cardiovascular disease in Bangladesh: Prospective cohort study. Bmj, 342(7806), 1-11. https://doi.org/10.1136/bmj.d2431

Cheng, H. Y., Li, P., David, M., Smithgall, T. E., Feng, L., \& Lieberman, M. W. (2004). Arsenic inhibition of the JAK-STAT pathway. Oncogene, 23(20), 3603-3612. https://doi.org/10.1038/sj.onc.1207466

Chung, J. R., Flannery, B., Thompson, M. G., Gaglani, M., Jackson, M. L., Monto, A. S., ... Fry, A. M. (2016). Seasonal Effectiveness of Live Attenuated and Inactivated Influenza Vaccine. Pediatrics, 137(2), e20153279-e20153279. https://doi.org/10.1542/peds.2015-3279 
Chung, J. Y., Yu, S. Do, \& Hong, Y. S. (2014). Environmental source of arsenic exposure. Journal of Preventive Medicine and Public Health, 47(5), 253-257. https://doi.org/10.3961/jpmph.14.036

Clara, M., Achaval, F., Arbiza, J., Blanc, A., Ruchansky, D., \& Le Bas, A. (2013). Serologic Evidence of Influenza A and B Viruses in South American Fur Seals (Arctocephalus australis). Journal of Wildlife Diseases, 45(2), 519-521. https://doi.org/10.7589/0090-355845.2.519

Cockburn, W. C., Delon, P. J., \& Ferreira, W. (1969). Origin and progress of the 1968-69 Hong Kong influenza epidemic. Bulletin of the World Health Organization, 41(3), 345-348.

Cooper, J. R., Abdullatif, M. B., Burnett, E. C., Kempsell, K. E., Conforti, F., Tolley, H., ... Davies, D. E. (2016). Long term culture of the a549 cancer cell line promotes multilamellar body formation and differentiation towards an alveolar type II Pneumocyte phenotype. PLoS ONE, 11(10), 1-20. https://doi.org/10.1371/journal.pone.0164438

Dangleben, N. L., Skibola, C. F., \& Smith, M. T. (2013). Arsenic immunotoxicity: A review. Environmental Health: A Global Access Science Source, 12(1), 1. https://doi.org/10.1186/1476-069X-12-73

Davoli, M., De Sario, M., Michelozzi, P., D’Ippoliti, D., Scortichini, M., \& Santelli, E. (2015). Arsenic in Drinking Water and Mortality for Cancer and Chronic Diseases in Central Italy, 1990-2010. Plos One, 10(9), e0138182. https://doi.org/10.1371/journal.pone.0138182

De Graaf, M., \& Fouchier, R. A. M. (2014). Role of receptor binding specificity in influenza A virus transmission and pathogenesis. EMBO Journal, 33(8), 823-841. https://doi.org/10.1002/embj.201387442

De Vlugt, C., Sikora, D., \& Pelchat, M. (2018). Insight into Influenza: A Virus Cap-Snatching. 
Viruses, 10(11), 641. https://doi.org/10.3390/v10110641

Devasthanam, A. S. (2014). Mechanisms underlying the inhibition of interferon signaling by viruses. Virulence, 5(2), 270-277. https://doi.org/10.4161/viru.27902

Di Pietro, A., Kajaste-Rudnitski, A., Oteiza, A., Nicora, L., Towers, G. J., Mechti, N., \& Vicenzi, E. (2013). TRIM22 Inhibits Influenza A Virus Infection by Targeting the Viral Nucleoprotein for Degradation. Journal of Virology, 87(8), 4523-4533. https://doi.org/10.1128/jvi.02548-12

Dong, J., \& Su, S. Y. (2009). The association between arsenic and children's intelligence: A meta-analysis. Biological Trace Element Research, 129(1-3), 88-93. https://doi.org/10.1007/s12011-008-8298-1

Dong, W., Wei, X., Zhang, F., Hao, J., Huang, F., Zhang, C., \& Liang, W. (2014). A dual character of flavonoids in influenza A virus replication and spread through modulating cellautonomous immunity by MAPK signaling pathways. Scientific Reports, 4, 1-12. https://doi.org/10.1038/srep07237

Dotis, J., \& Roilides, E. (2009). H1N1 influenza A infection. Hippokratia, 13(3), 135-8. Retrieved from http://www.ncbi.nlm.nih.gov/pubmed/19918299\%0Ahttp://www.pubmedcentral.nih.gov/art iclerender.fcgi?artid=PMC2765289

Dou, D., Revol, R., Östbye, H., Wang, H., \& Daniels, R. (2018). Influenza A virus cell entry, replication, virion assembly and movement. Frontiers in Immunology, 9(JUL), 1-17. https://doi.org/10.3389/fimmu.2018.01581

Doyle, D. (2009). Notoriety to respectability: A short history of arsenic prior to its present day use in haematology. British Journal of Haematology, 145(3), 309-317. 
https://doi.org/10.1111/j.1365-2141.2009.07623.x

Doyle, T. J., Goodin, K., \& Hamilton, J. J. (2013). Maternal and Neonatal Outcomes among Pregnant Women with 2009 Pandemic Influenza A(H1N1) Illness in Florida, 2009-2010: A Population-Based Cohort Study. PLoS ONE, 8(10), 2009-2010. https://doi.org/10.1371/journal.pone.0079040

Dozza, B., Smith, M. A., Perry, G., Tabaton, M., \& Strocchi, P. (2004). Regulation of glycogen synthase kinase- $3 \beta$ by products of lipid peroxidation in human neuroblastoma cells. Journal of Neurochemistry, 89(5), 1224-1232. https://doi.org/10.1111/j.1471-4159.2004.02413.x

Duarte, A. A. L. S., Cardoso, S. J. A., \& Alçada, A. J. (2009). Emerging and innovative techniques for arsenic removal applied to a small water supply system. Sustainability, 1(4), 1288-1304. https://doi.org/10.3390/su1041288

Ducatez, M. F., Pelletier, C., \& Meyer, G. (2015). Influenza d virus in cattle, France, 2011-2014. Emerging Infectious Diseases, 21(2), 368-371. https://doi.org/10.3201/eid2102.141449

Ehrhardt, C., \& Ludwig, S. (2009). A new player in a deadly game: Influenza viruses and the PI3K/Akt signalling pathway. Cellular Microbiology, 11(6), 863-871. https://doi.org/10.1111/j.1462-5822.2009.01309.x

Ehrhardt, C., Marjuki, H., Wolff, T., Nürnberg, B., Planz, O., Pleschka, S., \& Ludwig, S. (2006). Bivalent role of the phosphatidylinositol-3-kinase (PI3K) during influenza virus infection and host cell defence. Cellular Microbiology, 8(8), 1336-1348. https://doi.org/10.1111/j.1462-5822.2006.00713.x

Elderfield, R. A., Koutsakos, M., Frise, R., Bradley, K., Ashcroft, J., Miah, S., ... Barclay, W. S. (2016). NB protein does not affect influenza B virus replication in vitro and is not required for replication in or transmission between ferrets. Journal of General Virology, 97(3), 593- 
601. https://doi.org/10.1099/jgv.0.000386

Epperson, W. B., Olivier, A. K., Ferguson, L., Genova, S., Smith, D. R., Webby, R. J., ... Wan, X.-F. (2016). Pathogenesis of Influenza D Virus in Cattle. Journal of Virology, 90(12), 5636-5642. https://doi.org/10.1128/jvi.03122-15

Fabian, P., McDevitt, J. J., DeHaan, W. H., Fung, R. O. P., Cowling, B. J., Chan, K. H., ,.. Milton, D. K. (2008). Influenza Virus in Human Exhaled Breath: An Observational Study. PLoS ONE, 3(7), e2691. https://doi.org/10.1371/journal.pone.0002691

Faita, F., Cori, L., Bianchi, F., \& Andreassi, M. G. (2013). Arsenic-induced genotoxicity and genetic susceptibility to arsenic-related pathologies. International Journal of Environmental Research and Public Health, 10(4), 1527-1546. https://doi.org/10.3390/ijerph10041527

Fang, X., Yu, S. X., Lu, Y., Bast, R. C., Woodgett, J. R., \& Mills, G. B. (2000). Phosphorylation and inactivation of glycogen synthase kinase 3 by protein kinase A. Proceedings of the National Academy of Sciences, 97(22), 11960-11965. https://doi.org/10.1073/pnas.220413597

Farzan, S. F., Li, Z., Korrick, S. A., Spiegelman, D., Enelow, R., Nadeau, K., ... Karagas, M. R. (2016). Infant infections and respiratory symptoms in relation to in utero arsenic exposure in a U.S. cohort. Environmental Health Perspectives, 124(6), 840-847. https://doi.org/10.1289/ehp.1409282

Fischer, W. A., Gong, M., Bhagwanjee, S., \& Sevransky, J. (2014). Global burden of Influenza: Contributions from Resource Limited and Low-Income Settings. Global Heart, 9(3), 32536. https://doi.org/10.1016/j.gheart.2014.08.004

Flanagan, S. V., Johnston, R. B., \& Zheng, Y. (2012). Aarsenic dans l'eau des puits tubulaires au Bangladesh: Impacts sanitaires et économiques, et implications en vue de sa réduction. 
Bulletin of the World Health Organization, 90(11), 839-846.

https://doi.org/10.2471/BLT.11.101253

Food and Drug Administration (FDA). (2013). Draft Guidance for Industry Arsenic in Apple Juice : Action Level Draft Guidance. U.S. Department of Health and Human Services Food and Drug Administration Center for Food Safety and Applied Nutrition, (July 2013).

Frieden, T. R., Harold Jaffe, D. W., Director for Science James Stephens, A. W., Thacker, S. B., Casey, C. G., Holtzman, D., ... Sheryl Lyss, W. B. (2010). Centers for Disease Control and Prevention Morbidity and Mortality Weekly. Mmwr, 59(33), 1976-2007. Retrieved from https://www.cdc.gov/mmwr/pdf/wk/mm5933.pdf

Fukushima, K., Takahashi, T., Ito, S., Takaguchi, M., Takano, M., Kurebayashi, Y., ... Suzuki, T. (2014). Terminal sialic acid linkages determine different cell infectivities of human parainfluenza virus type 1 and type 3. Virology, 464-465(1), 424-431. https://doi.org/10.1016/j.virol.2014.07.033

Gamblin, S. J., \& Skehel, J. J. (2010). Influenza hemagglutinin and neuraminidase membrane glycoproteins. Journal of Biological Chemistry, 285(37), 28403-28409. https://doi.org/10.1074/jbc.R110.129809

Ganapathy, S., Liu, J., Xiong, R., Yu, T., \& Makriyannis, A. (2018). Chronic low dose arsenic exposure preferentially perturbs mitotic phase of the cell cycle. Genes \& Cancer, 10 . https://doi.org/10.18632/genesandcancer.185

García-Esquinas, E., Pollán, M., Umans, J. G., Francesconi, K. A., Goessler, W., Guallar, E., ... Navas-Acien, A. (2013). Arsenic exposure and cancer mortality in a US-based prospective cohort: the strong heart study. Cancer Epidemiology, Biomarkers \& Prevention : A Publication of the American Association for Cancer Research, Cosponsored by the 
American Society of Preventive Oncology, 22(11), 1944-53. https://doi.org/10.1158/10559965.EPI-13-0234-T

García-Sastre, A. (2010). Influenza virus receptor specificity: Disease and transmission. American Journal of Pathology, 176(4), 1584-1585. https://doi.org/10.2353/ajpath.2010.100066

Gaush, C. R., \& Smith, T. F. (1968). Replication and Plaque Assay of Influenza Virus in an Established Line of Canine Kidney Cells. Applied Microbiology, 16(4), 588-594.

Gazdar, A. F., Girard, L., Lockwood, W. W., Lam, W. L., \& Minna, J. D. (2010). Lung cancer cell lines as tools for biomedical discovery and research. Journal of the National Cancer Institute, 102(17), 1310-1321. https://doi.org/10.1093/jnci/djq279

George, C. M., Brooks, W. A., Graziano, J. H., Nonyane, B. A. S., Hossain, L., Goswami, D., ... O’ Brien, K. L. (2015). Arsenic exposure is associated with pediatric pneumonia in rural Bangladesh: a case control study. Environmental Health, 14(1), 83. https://doi.org/10.1186/s12940-015-0069-9

Gera, R., Singh, V., Mitra, S., Sharma, A. K., Singh, A., Dasgupta, A., .. Ghosh, D. (2017). Arsenic exposure impels CD4 commitment in thymus and suppress T cell cytokine secretion by increasing regulatory T cells. Scientific Reports, 7(1), 1-13. https://doi.org/10.1038/s41598-017-07271-z

Ghosh, J., \& Sil, P. C. (2015). Mechanism for Arsenic-Induced Toxic Effects. Handbook of Arsenic Toxicology. Elsevier Inc. https://doi.org/10.1016/B978-0-12-418688-0.00008-3

Ghosh, N., Das, S., Sil, A., Lahiri, S., Smith, A. H., Hira-Smith, M., ... Haque, R. (2005). Decrements in Lung Function Related to Arsenic in Drinking Water in West Bengal, India. American Journal of Epidemiology, 162(6), 533-541. https://doi.org/10.1093/aje/kwi236 
Golovkine, G., Faudry, E., Bouillot, S., Elsen, S., Attrée, I., \& Huber, P. (2016). Pseudomonas aeruginosa Transmigrates at Epithelial Cell-Cell Junctions, Exploiting Sites of Cell Division and Senescent Cell Extrusion. PLoS Pathogens, 12(1), 1-22. https://doi.org/10.1371/journal.ppat.1005377

Gomez-Puertas, P., Albo, C., Perez-Pastrana, E., Vivo, A., \& Portela, A. (2002). Influenza Virus Matrix Protein Is the Major Driving Force in Virus Budding. Journal of Virology, 74(24), 11538-11547. https://doi.org/10.1128/jvi.74.24.11538-11547.2000

Gonzalez, S., \& Ortin, J. (1999). Characterization of Influenza Virus PB1 Protein Binding to Viral RNA: Two Separate Regions of the Protein Contribute to the Interaction Domain. Journal of Virology, 73(1), 631-637.

Grais, R. F., Viboud, C., Lafont, B. A. P., Miller, M. A., \& Simonsen, L. (2005). Multinational Impact of the 1968 Hong Kong Influenza Pandemic: Evidence for a Smoldering Pandemic. The Journal of Infectious Diseases, 192(2), 233-248. https://doi.org/10.1086/431150

Guha Mazumder, D., \& Dasgupta, U. B. (2011). Chronic arsenic toxicity: Studies in West Bengal, India. Kaohsiung Journal of Medical Sciences, 27(9), 360-370. https://doi.org/10.1016/j.kjms.2011.05.003

Guo, X., Snider, W. D., \& Chen, B. (2016). Gsk3 $\beta$ regulates AKT-induced central nervous system axon regeneration via an $\mathrm{eIF} 2 \mathrm{~B} \varepsilon$-dependent, mTORC1-independent pathway. ELife, 5(MARCH2016), 1-18. https://doi.org/10.7554/eLife.11903

H.-R., G., N.-S., W., H., H., \& R.R., M. (2004). Cell type specificity of lung cancer associated with arsenic ingestion. Cancer Epidemiology Biomarkers and Prevention, 13(4), 638-643. Retrieved from http://www.embase.com/search/results?subaction=viewrecord\&from=export\&id=L3873790 
1\%0Ahttp://sfx.library.uu.nl/utrecht?sid=EMBASE\&issn=10559965\&id=doi:\&atitle=Cell+ type + specificity + of + lung + cancer + associated + with + arsenic + ingestion\&stitle $=$ Cancer + Epid emiol.+B

Ha, Y., Stevens, D. J., Skehel, J. J., \& Wiley, D. C. (2002). X-ray structures of H5 avian and H9 swine influenza virus hemagglutinins bound to avian and human receptor analogs. Proceedings of the National Academy of Sciences, 98(20), 11181-11186. https://doi.org/10.1073/pnas.201401198

Hale, B. G., Randall, R. E., Ortin, J., \& Jackson, D. (2008). The multifunctional NS1 protein of influenza A viruses. Journal of General Virology, 89(10), 2359-2376. https://doi.org/10.1099/vir.0.2008/004606-0

Haller, O., \& Kochs, G. (2002). <Traffic 2002 Haller.pdf>, (1), 710-717.

Hanika, A., Larisch, B., Steinmann, E., Schwegmann-Weßels, C., Herrler, G., \& Zimmer, G. (2005). Use of influenza C virus glycoprotein HEF for generation of vesicular stomatitis virus pseudotypes. Journal of General Virology, 86(5), 1455-1465. https://doi.org/10.1099/vir.0.80788-0

Hatta, M., Goto, H., \& Kawaoka, Y. (2004). Influenza B Virus Requires BM2 Protein for Replication. Journal of Virology, 78(11), 5576-5583. https://doi.org/10.1128/jvi.78.11.5576-5583.2004

Hatta, M., \& Kawaoka, Y. (2003). The NB Protein of Influenza B Virus Is Not Necessary for Virus Replication In Vitro. Journal of Virology, 77(10), 6050-6054. https://doi.org/10.1128/jvi.77.10.6050-6054.2003

Hayden, F. G., Vrooman, P. S., Barbarash, R., Bettis, R., Ward, P., \& Mills, R. G. (2000). Efficacy and Safety of the Oral Neuraminidase Inhibitor Oseltamivir in Treating Acute 
Influenza, 283(8).

He, W., Greenwell, R. J., Brooks, D. M., Calderón-Garcidueñas, L., Beall, H. D., \& Coffin, J. D. (2007). Arsenic exposure in pregnant mice disrupts placental vasculogenesis and causes spontaneous abortion. Toxicological Sciences, 99(1), 244-253. https://doi.org/10.1093/toxsci/kfm162

Hemmati, A. A., Alboghobeish, S., \& Ahangarpour, A. (2018). Chronic exposure to high fat diet exacerbates arsenic-induced lung damages in male mice: Possible role for oxidative stress. Monaldi Archives for Chest Disease, 88(1), 61-66.

https://doi.org/10.4081/monaldi.2018.903

Henderson, M. W., Madenspacher, J. H., Whitehead, G. S., Thomas, S. Y., Aloor, J. J., Gowdy, K. M., \& Fessler, M. B. (2017). Effects of orally ingested arsenic on respiratory epithelial permeability to bacteria and small molecules in mice. Environmental Health Perspectives, 125(9), 1-11. https://doi.org/10.1289/EHP1878

Hermann, A. C., \& Kim, C. H. (2005). Effects of arsenic on zebrafish innate immune system. Marine Biotechnology, 7(5), 494-505. https://doi.org/10.1007/s10126-004-4109-7

Hinman, A. R., \& Craven, R. B. (1978). Its History and for This Year' s Influenza Season. World Health, (December 2010).

Holmes, E. C., Zhan, Y., Wu, S., Lam, T. T.-Y., Guan, W., Pan, S., .. Tan, Y. (2013). Differing Epidemiological Dynamics of Influenza B Virus Lineages in Guangzhou, Southern China, 2009-2010. Journal of Virology, 87(22), 12447-12456. https://doi.org/10.1128/jvi.01039-13

Hong, Y.-S., Song, K.-H., \& Chung, J.-Y. (2014). Health effects of chronic arsenic exposure. $J$ Prev Med Public Health, 47(5), 245-52. https://doi.org/10.3961/jpmph.14.035

Hu, Y. W., Zhang, J., Wu, X. M., Cao, L., Nie, P., \& Chang, M. X. (2018). TANK-binding 
kinase 1 (TBK1) isoforms negatively regulate type I interferon induction by inhibiting TBK1-IRF3 interaction and IRF3 phosphorylation. Frontiers in Immunology, 9(JAN), 1-17. https://doi.org/10.3389/fimmu.2018.00084

Huang, J., Tong, D., Kim, C., Gu, L., Li, G.-M., \& Ortega, J. (2015). Arsenic Inhibits DNA Mismatch Repair by Promoting EGFR Expression and PCNA Phosphorylation. Journal of Biological Chemistry, 290(23), 14536-14541. https://doi.org/10.1074/jbc.m115.641399 Hubaux, R., Becker-Santos, D. D., Enfield, K. S. S., Rowbotham, D., Lam, S., Lam, W. L., \& Martinez, V. D. (2013). Molecular features in arsenic-induced lung tumors. Molecular Cancer, 12(1), 1-11. https://doi.org/10.1186/1476-4598-12-20

Hughes, M. F., Beck, B. D., Chen, Y., Lewis, A. S., \& Thomas, D. J. (2011). Arsenic exposure and toxicology: A historical perspective. Toxicological Sciences, 123(2), 305-332. https://doi.org/10.1093/toxsci/kfr184

Hundal, H. S., Singh, K., \& Singh, D. (2009). Arsenic content in ground and canal waters of Punjab, North-West India. Environmental Monitoring and Assessment, 154(1-4), 393-400. https://doi.org/10.1007/s10661-008-0406-3

Hussain, M., Galvin, H. D., Haw, T. Y., Nutsford, A. N., \& Husain, M. (2017). Drug resistance in influenza a virus: The epidemiology and management. Infection and Drug Resistance, 10(April), 121-134. https://doi.org/10.2147/IDR.S105473

Ibricevic, A., Pekosz, A., Walter, M. J., Newby, C., Battaile, J. T., Brown, E. G., ... Brody, S. L. (2006). Influenza virus receptor specificity and cell tropism in mouse and human airway epithelial cells. Journal of Virology, 80(15), 7469-80. https://doi.org/10.1128/JVI.02677-05 Ilyushina, N. A., Ikizler, M. R., Kawaoka, Y., Rudenko, L. G., Treanor, J. J., Subbarao, K., \& Wright, P. F. (2012). Comparative Study of Influenza Virus Replication in MDCK Cells 
and in Primary Cells Derived from Adenoids and Airway Epithelium. Journal of Virology, 86(21), 11725-11734. https://doi.org/10.1128/JVI.01477-12

Itagaki, T., Katsushima, N., Nishimura, H., Kitaoka, S., Matsuzaki, Y., Sakamoto, M., ... Nagai, Y. (2006). Clinical Features of Influenza C Virus Infection in Children. The Journal of Infectious Diseases, 193(9), 1229-1235. https://doi.org/10.1086/502973

Ito, T., \& Kawaoka, Y. (2000). Host-range barrier of influenza A viruses. Veterinary Microbiology, 74(1-2), 71-75. https://doi.org/10.1016/S0378-1135(00)00167-X

Ito, T., Suzuki, Y., Takada, A., Kawamoto, A., Otsuki, K., Masuda, H., ... Kawaoka, Y. (1997). Differences in sialic acid-galactose linkages in the chicken egg amnion and allantois influence human influenza virus receptor specificity and variant selection. Journal of Virology, 71(4), 3357-62. Retrieved from http://www.ncbi.nlm.nih.gov/pubmed/9060710\%0Ahttp://www.pubmedcentral.nih.gov/arti clerender.fcgi?artid=PMC191479

Jamieson, D. J., Honein, M. A., Rasmussen, S. A., Williams, J. L., Swerdlow, D. L., Biggerstaff, M. S., .. Wu, K. H. (2009). H1N1 2009 influenza virus infection during pregnancy in the USA. The Lancet, 374(9688), 451-458. https://doi.org/10.1016/S0140-6736(09)61304-0

Jang, J. H., Shin, H. W., Lee, J. M., Lee, H. W., Kim, E. C., \& Park, S. H. (2015). An Overview of Pathogen Recognition Receptors for Innate Immunity in Dental Pulp. Mediators of Inflammation, 2015. https://doi.org/10.1155/2015/794143

Jaspers, I., Ciencewicki, J. M., Zhang, W., Brighton, L. E., Carson, J. L., Beck, M. A., \& Madden, M. C. (2005). Diesel exhaust enhances influenza virus infections in respiratory epithelial cells. Toxicological Sciences, 85(2), 990-1002.

https://doi.org/10.1093/toxsci/kfi141 
Jiang, W. M., Wang, S. C., Peng, C., Yu, J. M., Zhuang, Q. Y., Hou, G. Y., ... Chen, J. M. (2014). Identification of a potential novel type of influenza virus in Bovine in China. Virus Genes, 49(3), 493-496. https://doi.org/10.1007/s11262-014-1107-3

Johnson, N. P. A. S., \& Mueller, J. (2007). Updating the Accounts: Global Mortality of the 19181920 \&quot;Spanish\&quot; Influenza Pandemic. Bulletin of the History of Medicine, 76(1), 105-115. https://doi.org/10.1353/bhm.2002.0022

Kesic, M. J., Meyer, M., Bauer, R., \& Jaspers, I. (2012). Exposure to ozone modulates human airway protease/antiprotease balance contributing to increased influenza a infection. PLoS ONE, 7(4), 1-12. https://doi.org/10.1371/journal.pone.0035108

Kilbourne, E. D. (2006). Influenza Pandemics of the 20th Century-Volume 12, Number 1January 2006-Emerging Infectious Disease journal-CDC, 12(1), 9-14.

Kile, M. L., Andres Houseman, E., Baccarelli, A. A., Quamruzzaman, Q., Rahman, M., Mostofa, G., ... Christiani, D. C. (2014). Effect of prenatal arsenic exposure on DNA methylation and leukocyte subpopulations in cord blood. Epigenetics, 9(5), 774-782. https://doi.org/10.4161/epi.28153

Kimble, B., Nieto, G. R., \& Perez, D. R. (2010). Characterization of influenza virus sialic acid receptors in minor poultry species. Virology Journal, 7(1), 365. https://doi.org/10.1186/1743-422X-7-365

Knight, M., Pierce, M., Seppelt, I., Kurinczuk, J. J., Spark, P., Brocklehurst, P., ... Sullivan, E. (2011). Critical illness with AH1N1v influenza in pregnancy: A comparison of two population-based cohorts. BJOG: An International Journal of Obstetrics and Gynaecology, 118(2), 232-239. https://doi.org/10.1111/j.1471-0528.2010.02736.x

Kozul, C. D., Ely, K. H., Enelow, R. I., \& Hamilton, J. W. (2009). Low-dose arsenic 
compromises the immune response to influenza $\mathrm{A}$ infection in vivo. Environmental Health Perspectives, 117(9), 1441-1447. https://doi.org/10.1289/ehp.0900911

Kozul, C. D., Hampton, T. H., Davey, J. C., Gosse, J. A., Nomikos, A. P., Eisenhauer, P. L., ... Hamilton, J. W. (2009). Chronic exposure to arsenic in the drinking water alters the expression of immune response genes in mouse lung. Environmental Health Perspectives, 117(7), 1108-1115. https://doi.org/10.1289/ehp.0800199

Kozul, C. D., Nomikos, A. P., Hampton, T. H., Warnke, L. A., Gosse, J. A., Davey, J. C., ... Hamilton, J. W. (2008). Laboratory diet profoundly alters gene expression and confounds genomic analysis in mouse liver and lung. Chemico-Biological Interactions, 173(2), 12940. https://doi.org/10.1016/j.cbi.2008.02.008

Kozul CD, E. K. E. R. H. J. (2009). Low-Dose Arsenic Compromises the Immune Response to Influenza A Infection in Vivo. Environ Health Perspect. 2009 Sep;117(9):1441-7. Doi: 10.1289/Ehp.0900911. Epub 2009 May 20., 117(9), 1441-7. Retrieved from http://www.ncbi.nlm.nih.gov/pmc/articles/PMC2737023/

Kulshrestha, A. (2014). Arsenic-induced abnormalities in glucose metabolism: Biochemical basis and potential therapeutic and nutritional interventions. World Journal of Translational Medicine, 3(2), 96. https://doi.org/10.5528/wjtm.v3.i2.96

Kumar, A., Rahman, M. S., Iqubal, M. A., Ali, M., Niraj, P. K., Anand, G., ... Ghosh, A. K. (2016). Ground water arsenic contamination: A local survey in India. International Journal of Preventive Medicine, 2016August(August). https://doi.org/10.4103/2008-7802.188085

Kumar, S., Brown, A., \& Tchounwou, P. B. (2018). Trisenox disrupts MDM2-DAXX-HAUSP complex and activates p53, cell cycle regulation and apoptosis in acute leukemia cells. Oncotarget, 9(69), 33138-33148. https://doi.org/10.18632/oncotarget.26025 
Kunisaki, K. M., \& Janoff, E. N. (2009). Influenza in immunosuppressed populations: a review of infection frequency, morbidity, mortality, and vaccine responses. The Lancet Infectious Diseases, 9(8), 493-504. https://doi.org/10.1016/S1473-3099(09)70175-6

Kuo, C., Moon, K. A., Wang, S., Silbergeld, E., \& Navas-acien, A. (2013). The Association of Arsenic Metabolism with Cancer, Cardiovascular Disease, and Diabetes : A Systematic Review of the Epidemiological Evidence. https://doi.org/10.1289/EHP577

Kuss-Duerkop, S. K., Wang, J., Mena, I., White, K., Metreveli, G., Sakthivel, R., ... Fontoura, B. M. A. (2017). Influenza virus differentially activates mTORC1 and mTORC2 signaling to maximize late stage replication. PLoS Pathogens (Vol. 13). https://doi.org/10.1371/journal.ppat.1006635

Lage, C. R., Nayak, A., \& Kim, C. H. (2006). Arsenic ecotoxicology and innate immunity. Integrative and Comparative Biology, 46(6), 1040-1054. https://doi.org/10.1093/icb/icl048

Lai, M. W. (2005). Acute Arsenic Poisoning in Two Siblings. Pediatrics, 116(1), 249-257. https://doi.org/10.1542/peds.2004-1957

Lau, A., Whitman, S. A., Jaramillo, M. C., \& Zhang, D. D. (2013). Arsenic-Mediated Activation of the Nrf2-Keap1 Antioxidant Pathway. Journal of Biochemical and Molecular Toxicology, 27(2), 99-105. https://doi.org/10.1002/jbt.21463

Lee, A. J., \& Ashkar, A. A. (2018). The dual nature of type I and type II interferons. Frontiers in Immunology, 9(SEP), 1-10. https://doi.org/10.3389/fimmu.2018.02061

Lee, E., Gribble, M. O., Best, L. G., Kuo, C.-C., Goessler, W., Umans, J. G., ... Navas-Acien, A. (2015). Arsenic Exposure, Arsenic Metabolism, and Incident Diabetes in the Strong Heart Study. Diabetes Care, 38(April), dc141641. https://doi.org/10.2337/dc14-1641

Lee, P. C., Ho, I. C., \& Lee, T. C. (2005). Oxidative stress mediates sodium arsenite-induced 
expression of heme oxygenase-1, monocyte chemoattractant protein-1, and interleukin-6 in vascular smooth muscle cells. Toxicological Sciences, 85(1), 541-550. https://doi.org/10.1093/toxsci/kfi101

Li, W., Wang, G., Zhang, H., Shen, Y., Dai, J., Wu, L., ... Li, K. (2012). Inability of NS1 protein from an $\mathrm{H} 5 \mathrm{~N} 1$ influenza virus to activate PI3K/Akt signaling pathway correlates to the enhanced virus replication upon PI3K inhibition. Veterinary Research, 43(1), 1-12. https://doi.org/10.1186/1297-9716-43-36

Lin, S. C., Kappes, M. A., Chen, M. C., Lin, C. C., \& Wang, T. T. (2017). Distinct susceptibility and applicability of MDCK derivatives for influenza virus research. PLoS ONE, 12(2), 112. https://doi.org/10.1371/journal.pone.0172299

Lin, Y., Zhuang, L., Yi, H., Xu, L., Huang, H., He, D., ... Wu, L. (2018). Embryonic protective role of folate in arsenic-induced cardiac malformations in rats, 11(4), 1946-1955.

Littauer, E. Q., Esser, E. S., Antao, O. Q., Vassilieva, E. V., Compans, R. W., \& Skountzou, I. (2017). H1N1 influenza virus infection results in adverse pregnancy outcomes by disrupting tissue-specific hormonal regulation. PLoS Pathogens, 13(11), 1-27. https://doi.org/10.1371/journal.ppat.1006757

Liu, J., \& Waalkes, M. P. (2008). Liver is a target of arsenic carcinogenesis. Toxicological Sciences, 105(1), 24-32. https://doi.org/10.1093/toxsci/kfn120

Livak, K. J., \& Schmittgen, T. D. (2001). Analysis of Relative Gene Expression Data Using Real- Time Quantitative PCR and the 2 X 00 C T Method, 408, 402-408. https://doi.org/10.1006/meth.2001.1262

Long, J. C. D., \& Fodor, E. (2016). The PB2 Subunit of the Influenza A Virus RNA Polymerase 
Is Imported into the Mitochondrial Matrix. Journal of Virology, 90(19), 8729-8738. https://doi.org/10.1128/jvi.01384-16

Luna, A. L., Soto-Peña, G. A., Vega, L., Cebrián, M. E., López-Carrillo, L., Conde, P., ... Bastida, M. (2006). Assessment of lymphocyte subpopulations and cytokine secretion in children exposed to arsenic. The FASEB Journal, 20(6), 779-781. https://doi.org/10.1096/fj.05-4860fje

Luo, X., Guo, L., Zhang, J., Xu, Y., Gu, W., Feng, L., \& Wang, Y. (2017). Tight Junction Protein Occludin Is a Porcine Epidemic Diarrhea Virus Entry Factor. Journal of Virology, 91(10), 1-14. https://doi.org/10.1128/jvi.00202-17

Ma, W., Kahn, R. E., \& Richt, J. A. (2009). The pig as a mixing vessel for influenza viruses... $J$ Mol Genet Med, 3(1), 158-166. Retrieved from www.google.org

MacDonald, N., \& Bortolussi, R. (2009). Protecting young babies from influenza. Paediatrics and Child Health, 14(9), 612-614. https://doi.org/10.1093/pch/14.9.612

Maelfait, J., Roose, K., Vereecke, L., Mc Guire, C., Sze, M., Schuijs, M. J., ... van Loo, G. (2016). A20 Deficiency in Lung Epithelial Cells Protects against Influenza A Virus Infection. PLoS Pathogens, 12(1), 1-17. https://doi.org/10.1371/journal.ppat.1005410

Mair, C. M., Ludwig, K., Herrmann, A., \& Sieben, C. (2014). Receptor binding and pH stability - How influenza A virus hemagglutinin affects host-specific virus infection. Biochimica et Biophysica Acta - Biomembranes, 1838(4), 1153-1168. https://doi.org/10.1016/j.bbamem.2013.10.004

Manzoor, R., Igarashi, M., \& Takada, A. (2017). Influenza A virus M2 protein: Roles from ingress to egress. International Journal of Molecular Sciences, 18(12), 1-16. https://doi.org/10.3390/ijms18122649 
Mao, H., Tu, W., Qin, G., Law, H. K. W., Sia, S. F., Chan, P.-L., ... Lau, Y.-L. (2009). Influenza Virus Directly Infects Human Natural Killer Cells and Induces Cell Apoptosis. Journal of Virology, 83(18), 9215-9222. https://doi.org/10.1128/JVI.00805-09

Marcason, W. (2015). What Are the Current Findings Concerning Arsenic in Foods? Journal of the Academy of Nutrition and Dietetics, 115(6), 1028.

https://doi.org/10.1016/j.jand.2015.04.011

Marshall, V., \& Baylor, N. W. (2011). Food and Drug Administration Regulation and Evaluation of Vaccines. Pediatrics, 127(Supplement), S23-S30. https://doi.org/10.1542/peds.2010$1722 \mathrm{e}$

Martinez, V. D., Vucic, E. A., Becker-Santos, D. D., Gil, L., \& Lam, W. L. (2011). Arsenic exposure and the induction of human cancers. Journal of Toxicology, 2011. https://doi.org/10.1155/2011/431287

Matrosovich, M. N., Matrosovich, T. Y., Gray, T., Roberts, N. A., \& Klenk, H.-D. (2004). Human and avian influenza viruses target different cell types in cultures of human airway epithelium. Proceedings of the National Academy of Sciences, 101(13), 4620-4624. https://doi.org/10.1073/pnas.0308001101

Matsuoka, Y., Matsumae, H., Katoh, M., Eisfeld, A. J., Neumann, G., Hase, T., ... Kawaoka, Y. (2013). A comprehensive map of the influenza A virus replication cycle. BMC Systems Biology, 7(1), 1. https://doi.org/10.1186/1752-0509-7-97

McAuley, J. L., Zhang, K., \& McCullers, J. A. (2009). The Effects of Influenza A Virus PB1-F2 Protein on Polymerase Activity Are Strain Specific and Do Not Impact Pathogenesis. Journal of Virology, 84(1), 558-564. https://doi.org/10.1128/jvi.01785-09

McCarthy, A. A., Crépin, T., Hart, D. J., Ruigrok, R. W. H., Bouvier, D., Baudin, F., ... Cusack, 
S. (2009). The cap-snatching endonuclease of influenza virus polymerase resides in the PA subunit. Nature, 458(7240), 914-918. https://doi.org/10.1038/nature07745

Mccauley, J. W., \& Mahy, B. W. J. (1983). REVIEW ARTICLE Structure and function of the influenza virus genome. Biochem J., 211, 281-294.

Mckimm-Breschkin, J. L. (2013). Influenza neuraminidase inhibitors: Antiviral action and mechanisms of resistance. Influenza and Other Respiratory Viruses, 7(1 SUPPL.1), 25-36. https://doi.org/10.1111/irv.12047

Milton, A. H., Hasan, Z., Rahman, A., \& Rahman, M. (2001). Chronic arsenic poisoning and respiratory effects in Bangladesh. Journal of Occupational Health, 43(3), 136-140. https://doi.org/10.1539/joh.43.136

Minatel, B. C., Sage, A. P., Anderson, C., Hubaux, R., Marshall, E. A., Lam, W. L., \& Martinez, V. D. (2018). Environmental arsenic exposure: From genetic susceptibility to pathogenesis. Environment International, 112(December 2017), 183-197. https://doi.org/10.1016/j.envint.2017.12.017

Mohammed Abdul, K. S., Jayasinghe, S. S., Chandana, E. P. S., Jayasumana, C., \& De Silva, P. M. C. S. (2015). Arsenic and human health effects: A review. Environmental Toxicology and Pharmacology, 40(3), 828-846. https://doi.org/10.1016/j.etap.2015.09.016

Moon, K. (Department of E. H. S., Exposure, A., \& Review, S. (2013). NIH Public Access, 14(6), 542-555. https://doi.org/10.1007/s11883-012-0280-x.Arsenic

Morens, D. M., \& Fauci, A. S. (2007). The 1918 Influenza Pandemic: Insights for the 21st Century. The Journal of Infectious Diseases, 195(7), 1018-1028. https://doi.org/10.1086/511989

Morens, D. M., Taubenberger, J. K., Harvey, H. A., \& Memoli, M. J. (2010). The 1918 influenza 
pandemic: Lessons for 2009 and the future. Critical Care Medicine, 38(SUPPL. 4). https://doi.org/10.1097/CCM.0b013e3181ceb25b

Mostofa, G., Christiani, D. C., Rahman, M. L., Ahmed, S. M., Joya, S. A., Ibn Hasan, M. O. S., ... Kile, M. L. (2018). A Prospective Cohort Study Examining the Associations of Maternal Arsenic Exposure With Fetal Loss and Neonatal Mortality. American Journal of Epidemiology, 188(2), 347-354. https://doi.org/10.1093/aje/kwy243

Muraki, Y., Okuwa, T., Himeda, T., Hongo, S., \& Ohara, Y. (2013). Effect of Cysteine Mutations in the Extracellular Domain of CM2 on the Influenza C Virus Replication. PLoS ONE, 8(4). https://doi.org/10.1371/journal.pone. 0060510

Nair, H., Brooks, W. A., Katz, M., Roca, A., Berkley, J. A., Madhi, S. A., ... Campbell, H. (2011). Global burden of respiratory infections due to seasonal influenza in young children: A systematic review and meta-analysis. The Lancet, 378(9807), 1917-1930. https://doi.org/10.1016/S0140-6736(11)61051-9

Namgung, U., \& Xia, Z. (2000). Arsenite-induced apoptosis in cortical neurons is mediated by cJun N-terminal protein kinase 3 and p38 mitogen-activated protein kinase. The Journal of Neuroscience: The Official Journal of the Society for Neuroscience, 20(17), 6442-51. Retrieved from http://www.ncbi.nlm.nih.gov/pubmed/10964950

NARUKAWA, T., IWAI, T., \& CHIBA, K. (2018). Determination of Inorganic Arsenic in Grape Products Using HPLC-ICP-MS. Analytical Sciences, 34(6), 687-691. https://doi.org/10.2116/analsci.18sbp02

Nayak, A. S., Lage, C. R., \& Kim, C. H. (2007). Effects of low concentrations of arsenic on the innate immune system of the zebrafish (Danio Rerio). Toxicological Sciences, 98(1), 118124. https://doi.org/10.1093/toxsci/kfm072 
Ni, F., Kondrashkina, E., \& Wang, Q. (2013). Structural basis for the divergent evolution of influenza B virus hemagglutinin. Virology, 446(1-2), 112-122. https://doi.org/10.1016/j.virol.2013.07.035

Nicholls, J. M., Bourne, A. J., Chen, H., Guan, Y., \& Peiris, J. M. (2007). Sialic acid receptor detection in the human respiratory tract: Evidence for widespread distribution of potential binding sites for human and avian influenza viruses. Respiratory Research, 8, 1-10. https://doi.org/10.1186/1465-9921-8-73

Nichols, L., Saunders, R., \& Knollmann, F. D. (2012). Causes of death of patients with lung cancer. Archives of Pathology and Laboratory Medicine, 136(12), 1552-1557. https://doi.org/10.5858/arpa.2011-0521-OA

O’Neill, R. E., Talon, J., \& Palese, P. (1998). The influenza virus NEP (NS2 protein) mediates the nuclear export of viral ribonucleoproteins. EMBO Journal, 17(1), 288-296. https://doi.org/10.1093/emboj/17.1.288

Ohnuki, Y., Ke, Y., Gerwin, B. I., Lechner, J. F., \& Harris, C. C. (1993). Development of Tumorigenicity in Simian Virus 40-immortalized Human Bronchial Epithelial Cell Lines. Cancer Research, 53(5), 985-991.

Oner, A. F., Dogan, N., Gasimov, V., Adisasmito, W., Coker, R., Chan, P. K. S., .. Dreyer, N. A. (2012). H5N1 avian influenza in children. Clinical Infectious Diseases, 55(1), 26-32. https://doi.org/10.1093/cid/cis295

Otte, J., Roland-Holst, D., Pfeiffer, D., Soares-Magalhaes, R., Rushton, J., Graham, J., \& Silbergeld, E. (2007). Industrial livestock production and global health risks. Pro-Poor Livsetock Policy Initiative, (07), 1-21. Retrieved from http://www.dfid.gov.uk/r4d/PDF/Outputs/Livestock/PPLPIrep- 
hpai_industrialisationrisks.pdf

Palmieri, M., Lotfi, P., Bajaj, L., Bondar, V. V., Sanagasetti, D., Sardiello, M., ... Wu, S. M. (2017). MTORC1-independent TFEB activation via Akt inhibition promotes cellular clearance in neurodegenerative storage diseases. Nature Communications, 8(May 2016). https://doi.org/10.1038/ncomms14338

Pappas, G., Kiriaze, I. J., \& Falagas, M. E. (2008). Insights into infectious disease in the era of Hippocrates. International Journal of Infectious Diseases, 12(4), 347-350. https://doi.org/10.1016/j.ijid.2007.11.003

Parsons, P. J., Johnson, D. N., Seino, S., Hara, M., Ye, H., Ruiz, D., ... Kamau, W. (2017). Arsenic exposure induces glucose intolerance and alters global energy metabolism. American Journal of Physiology-Regulatory, Integrative and Comparative Physiology, 314(2), R294-R303. https://doi.org/10.1152/ajpregu.00522.2016

Parvez, F., Chen, Y., Brandt-Rauf, P. W., Bernard, A., Dumont, X., Slavkovich, V., ... Ahsan, H. (2008). Nonmalignant respiratory effects of chronic arsenic exposure from drinking water among never-smokers in Bangladesh. Environmental Health Perspectives, 116(2), 190-195. https://doi.org/10.1289/ehp.9507

Parvez, F., Chen, Y., Yunus, M., Olopade, M., Segers, S., Slavkovich, V., ... Ahsan, H. (2013). Arsenic exposure and impaired lung function findings from a large population-based prospective cohort study. American Journal of Respiratory and Critical Care Medicine, 188(7), 813-819. https://doi.org/10.1164/rccm.201212-2282OC

Peiris, J. S. M., De Jong, M. D., \& Guan, Y. (2007). Avian influenza virus (H5N1): A threat to human health. Clinical Microbiology Reviews, 20(2), 243-267. https://doi.org/10.1128/CMR.00037-06 
Peltola, V., Ziegler, T., \& Ruuskanen, O. (2003). Influenza A and B Virus Infections in Children. Clinical Infectious Diseases, 36(3), 299-305. https://doi.org/10.1086/345909

Pesola, G. R., Parvez, F., Chen, Y., Ahmed, A., Hasan, R., \& Ahsan, H. (2012). Arsenic exposure from drinking water and dyspnoea risk in Araihazar, Bangladesh: A populationbased study. European Respiratory Journal, 39(5), 1076-1083.

https://doi.org/10.1183/09031936.00042611

Pica, N., \& Bouvier, N. M. (2012). Environmental factors affecting the transmission of respiratory viruses. Current Opinion in Virology, 2(1), 90-95. https://doi.org/10.1016/j.coviro.2011.12.003

Potter, C. W. (2001). A history of influenza. Journal of Applied Microbiology, 91(4), 572-579. https://doi.org/10.1046/j.1365-2672.2001.01492.x

Proença-Módena, J. L., Macedo, I. S., \& Arruda, E. (2007). H5N1 avian influenza virus: an overview. Brazilian Journal of Infectious Diseases, 11(1), 125-133. https://doi.org/10.1590/s1413-86702007000100027

Putila, J. J., \& Guo, N. L. (2011). Association of arsenic exposure with lung cancer incidence rates in the United States. PLoS ONE, 6(10), 1-7. https://doi.org/10.1371/journal.pone.0025886

Qi, J., Chen, X., Shi, Y., Gao, G. F., Diaz, S., Song, H., ... Khedri, Z. (2016). An Open Receptor-Binding Cavity of Hemagglutinin-Esterase-Fusion Glycoprotein from NewlyIdentified Influenza D Virus: Basis for Its Broad Cell Tropism. PLOS Pathogens, 12(1), e1005411. https://doi.org/10.1371/journal.ppat.1005411

Racaniello, V. R., \& Palese, P. (1979). Isolation of influenza C virus recombinants. Journal of Virology, 32(3), 1006-14. Retrieved from 
http://www.ncbi.nlm.nih.gov/pubmed/513198\%0Ahttp://www.pubmedcentral.nih.gov/articl erender.fcgi?artid=PMC525950

Rahman, A., Vahter, M., Ekström, E. C., \& Persson, L. Å. (2011). Arsenic exposure in pregnancy increases the risk of lower respiratory tract infection and diarrhea during infancy in Bangladesh. Environmental Health Perspectives, 119(5), 719-724. https://doi.org/10.1289/ehp.1002265

Rahman, A., Vahter, M., Smith, A. H., Nermell, B., Yunus, M., El Arifeen, S., ... Ekström, E. C. (2009). Arsenic exposure during pregnancy and size at birth: A prospective cohort study in Bangladesh. American Journal of Epidemiology, 169(3), 304-312. https://doi.org/10.1093/aje/kwn332

Ramsey, K. A., Bosco, A., Mckenna, K. L., Carter, K. W., Elliot, J. G., Berry, L. J., ... Zosky, G. R. (2013). In utero exposure to arsenic alters lung development and genes related to immune and mucociliary function in mice. Environmental Health Perspectives, 121(2), 244-250. https://doi.org/10.1289/ehp.1205590

Ramsey, K. A., Foong, R. E., Sly, P. D., Larcombe, A. N., \& Zosky, G. R. (2013). Early life arsenic exposure and acute and long-term responses to influenza A infection in mice. Environmental Health Perspectives, 121(10), 1187-1193.

https://doi.org/10.1289/ehp.1306748

Reddel, R. R., Ke, Y., Gerwin, B. I., McMenamin, M. G., Lechner, J. F., Su, R. T., ... Harris, C. C. (1988). Transformation of Human Bronchial Epithelial Cells by Infection with SV40 or Adenovirus-12 SV40 Hybrid Virus, or Transfection via Strontium Phosphate Coprecipitation with a Plasmid Containing SV40 Early Region Genes. Cancer Research, 48(7), 1904-1909. 
Reimann, C., Matschullat, J., Birke, M., \& Salminen, R. (2009). Arsenic distribution in the environment: The effects of scale. Applied Geochemistry, 24(7), 1147-1167. https://doi.org/10.1016/j.apgeochem.2009.03.013

Rossman, J. S., \& Lamb, R. A. (2011). Influenza virus assembly and budding. Virology, 411(2), 229-236. https://doi.org/10.1016/j.virol.2010.12.003

Rozo, M., \& Gronvall, G. K. (2015). The Reemergent 1977 H1N1 Strain and the Gain-ofFunction Debate. MBio, 6(4), 1-6. https://doi.org/10.1128/mbio.01013-15

Ruf, B. R., \& Knuf, M. (2014). The burden of seasonal and pandemic influenza in infants and children. European Journal of Pediatrics, 173(3), 265-276. https://doi.org/10.1007/s00431013-2023-6

Salomon, R., Staeheli, P., Kochs, G., Yen, H. L., Franks, J., Rehg, J. E., ... Hoffmann, E. (2007). Mx1 gene protects mice against the highly lethal human H5N1 influenza virus. Cell Cycle, 6(19), 2417-2421. https://doi.org/10.4161/cc.6.19.4779

Samji, T. (2008). T . sonic 850 Firmware update note, 82, 2008.

Saunders-Hastings, P., \& Krewski, D. (2016). Reviewing the History of Pandemic Influenza: Understanding Patterns of Emergence and Transmission. Pathogens, 5(4), 66. https://doi.org/10.3390/pathogens5040066

Schanzer, D. L., Langley, J. M., \& Tam, T. W. S. (2007). Influenza-Attributed Hospitalization Rates Among Pregnant Women in Canada 1994-2000. Journal of Obstetrics and Gynaecology Canada, 29(8), 622-629. https://doi.org/10.1016/S1701-2163(16)32559-2

Schneider, W. M., Chevillotte, M. D., \& Rice, C. M. (2014). Interferon-Stimulated Genes: A Complex Web of Host Defenses. Annual Review of Immunology, 32(1), 513-545. https://doi.org/10.1146/annurev-immunol-032713-120231 
Schnell, D., Mayaux, J., de Bazelaire, C., Legoff, J., Feuillet, S., Scieux, C., ... Azoulay, É. (2010). Risk factors for pneumonia in immunocompromised patients with influenza. Respiratory Medicine, 104(7), 1050-1056. https://doi.org/10.1016/j.rmed.2010.01.021

Scull, M. A., \& Rice, C. M. (2010). A big role for small RNAs in influenza virus replication. Proceedings of the National Academy of Sciences, 107(25), 11153-11154. https://doi.org/10.1073/pnas.1006673107

Seleka, M., Treurnicht, F. K., Tempia, S., Hellferscee, O., Mtshali, S., Cohen, A. L., ... Venter, M. (2017). Epidemiology of influenza B/Yamagata and B/Victoria lineages in South Africa, 2005-2014. PLoS ONE, 12(5), 2005-2014. https://doi.org/10.1371/journal.pone.0177655

Seo, S. H., \& Webster, R. G. (2002). Tumor necrosis factor alpha exerts powerful anti-influenza virus effects in lung epithelial cells. Journal of Virology, 76(3), 1071-6. https://doi.org/10.1128/JVI.76.3.1071

Shen, S., Li, X., Cullen, W. R., Weinfeld, M., \& Le, X. C. (2013). Arsenic Binding to Proteins.

Sheng, Z., Hause, B. M., Chakravarty, S., Collin, E. A., Li, F., Webby, R. J., .. Liu, R. (2013). Isolation of a Novel Swine Influenza Virus from Oklahoma in 2011 Which Is Distantly Related to Human Influenza C Viruses. PLoS Pathogens, 9(2), e1003176. https://doi.org/10.1371/journal.ppat.1003176

Sherwood, C. L., Clark Lantz, R., \& Boitano, S. (2013). Chronic arsenic exposure in nanomolar concentrations compromises wound response and intercellular signaling in airway epithelial cells. Toxicological Sciences, 132(1), 222-234. https://doi.org/10.1093/toxsci/kfs331

Sherwood, C. L., Liguori, A. E., Olsen, C. E., Lantz, R. C., Burgess, J. L., \& Boitano, S. (2013). Arsenic compromises conducting airway epithelial barrier properties in primary mouse and immortalized human cell cultures. PLoS ONE, 8(12), 1-10. 
https://doi.org/10.1371/journal.pone.0082970

Shi, Y., Wu, Y., Zhang, W., Qi, J., \& Gao, G. F. (2014). Enabling the ' host jump ': structural determinants of receptor-binding specificity in influenza A viruses. Nature Publishing Group, 12(12), 822-831. https://doi.org/10.1038/nrmicro3362

Shooshtary, S., Behtash, S., \& Nafisi, S. (2015). Arsenic trioxide binding to serum proteins. Journal of Photochemistry and Photobiology B: Biology, 148, 31-36. https://doi.org/10.1016/j.jphotobiol.2015.03.001

Short, K. R., Kasper, J., Van Der Aa, S., Andeweg, A. C., Zaaraoui-Boutahar, F., Goeijenbier, M., ... Kuiken, T. (2016). Influenza virus damages the alveolar barrier by disrupting epithelial cell tight junctions. European Respiratory Journal, 47(3), 954-966. https://doi.org/10.1183/13993003.01282-2015

Shortridge, K. F., Gao, P., Guan, Y., Ito, T., Kawaoka, Y., Markwell, D., ... Webster, R. G. (2000). Interspecies transmission of influenza viruses: H5N1 virus and a Hong Kong SAR perspective. Veterinary Microbiology, 74(1-2), 141-147. https://doi.org/10.1016/S0378$1135(00) 00174-7$

Shrivastava, B. K. (2016). Policy intervention for arsenic mitigation in Drinking Water in rural habitations in India: Achievements and challenges. Journal of Water and Health, 14(5), 827-838. https://doi.org/10.2166/wh.2016.014

Smed-Sörensen, A., Chalouni, C., Chatterjee, B., Cohn, L., Blattmann, P., Nakamura, N., ... Mellman, I. (2012). Influenza a virus infection of human primary dendritic cells impairs their ability to cross-present antigen to CD8 T cells. PLoS Pathogens, 8(3). https://doi.org/10.1371/journal.ppat.1002572

Smith, A. H., Goycolea, M., Haque, R., \& Biggs, M. Lou. (1998). ! Region II. American Journal 
of Epidemiology, 147(7), 660-669. https://doi.org/10.1111/j.1468-2478.2011.00666.x

Smith, A. H., Hopenhayn-Rich, C., Bates, M. N., Goeden, H. M., Hertz-Picciotto, I., Duggan, H. M., ... Smith, M. T. (1992). Cancer risks from arsenic in drinking water. In Environmental Health Perspectives (Vol. 97, pp. 259-267). https://doi.org/10.1289/ehp.9297259

Smith, A. H., Lingas, E. O., \& Rahman, M. (2000). Contamination of Drinking Water by Arsenic in Bangladesh : A Public Health Emergency . Bulletin of the World Health Organization 78: Contamination of drinking-water by arsenic in Bangladesh : a public health emergency. World Health Organization. Bulletin of the World Health Organization, 78(August 2016), 1093. https://doi.org/10.1590/S0042-96862000000900005

Smith, A. H., Marshall, G., Roh, T., Ferreccio, C., Liaw, J., \& Steinmaus, C. (2018). Lung, bladder, and kidney cancer mortality 40 years after arsenic exposure reduction. Journal of the National Cancer Institute, 110(3), 241-249. https://doi.org/10.1093/jnci/djx201

Smith, A. H., Marshall, G., Yuan, Y., Ferreccio, C., Liaw, J., von Ehrenstein, O., ... Selvin, S. (2006). Increased mortality from lung cancer and bronchiectasis in young adults after exposure to arsenic in utero and in early childhood. Environmental Health Perspectives, 114(8), 1293-1296. https://doi.org/10.1289/ehp.8832

Smith, A. H., Yunus, M., Khan, A. F., Ercumen, A., Yuan, Y., Smith, M. H., ... Steinmaus, C. (2013). Chronic respiratory symptoms in children following in utero and early life exposure to arsenic in drinking water in Bangladesh. International Journal of Epidemiology, 42(4), 1077-1086. https://doi.org/10.1093/ije/dyt120

Stegemann-Koniszewski, S., Jeron, A., Gereke, M., Geffers, R., Kröger, A., \& Gunzer, M. (2016). Alveolar Type II Epithelial Cells Contribute to the Anti-Influenza A Virus Response in the Lung by Integrating Pathogen- and Microenvironmen-Derived Signals. 
MBio, 7(3), 1-11. https://doi.org/10.1128/mBio.00276-16.Editor

Steinmaus, C. M., Ferreccio, C., Romo, J. A., Yuan, Y., Cortes, S., Marshall, G., ... Smith, A. H. (2013). Drinking water arsenic in northern chile: High cancer risks 40 years after exposure cessation. Cancer Epidemiology Biomarkers and Prevention, 22(4), 623-630. https://doi.org/10.1158/1055-9965.EPI-12-1190

Steinmaus, C., Marshall, G., Bates, M., Liaw, J., Ferreccio, C., Smith, A. H., \& Yuan, Y. (2009). Kidney Cancer Mortality. Epidemiology, 21(1), 103-108. https://doi.org/10.1097/ede.0b013e3181c21e46

Stencel-Baerenwald, J. E., Reiss, K., Reiter, D. M., Stehle, T., \& Dermody, T. S. (2014). The sweet spot: Defining virus-sialic acid interactions. Nature Reviews Microbiology, 12(11), 739-749. https://doi.org/10.1038/nrmicro3346

Stewart, C. E., Torr, E. E., Mohd Jamili, N. H., Bosquillon, C., \& Sayers, I. (2012). Evaluation of Differentiated Human Bronchial Epithelial Cell Culture Systems for Asthma Research. Journal of Allergy, 2012(di), 1-11. https://doi.org/10.1155/2012/943982

Stifter, S. A., \& Feng, C. G. (2015). Interfering with Immunity: Detrimental Role of Type I IFNs during Infection. The Journal of Immunology, 194(6), 2455-2465. https://doi.org/10.4049/jimmunol.1402794

Stiver, G. (2003). The treatment of influenza with antiviral drugs. Cmaj, 168(1), 49-57.

Sufiawati, I., \& Tugizov, S. M. (2014). HIV-associated disruption of tight and adherens junctions of oral epithelial cells facilitates HSV-1 infection and spread. PLOS ONE, 9(2). https://doi.org/10.1371/journal.pone.0088803

Sun, H., Qu, L., Gu, M., Yang, Y., Jiang, L., Sun, W., ... Gao, Y. (2017). Sodium ArseniteInduced Learning and Memory Impairment Is Associated with Endoplasmic Reticulum 
Stress-Mediated Apoptosis in Rat Hippocampus. Frontiers in Molecular Neuroscience, 10(September), 1-14. https://doi.org/10.3389/fnmol.2017.00286

Sung, T. C., Huang, J. W., Guo, H. R., \& Su, S. Bin. (2015). Association between arsenic exposure and diabetes: A meta-analysis. BioMed Research International, 2015, 17-22. https://doi.org/10.1155/2015/368087

Swamy, M., Jamora, C., Havran, W., \& Hayday, A. (2010). Epithelial decision makers: In search of the "epimmunome." Nature Immunology, 11(8), 656-665. https://doi.org/10.1038/ni.1905

Sweet, C., Jakeman, K. J., Leone, A., Russell, S., \& Tisdale, M. (2012). Efficacy of 2'-deoxy-2'fluororibosides against influenza A and B viruses in ferrets. Antimicrobial Agents and Chemotherapy, 38(8), 1864-1867. https://doi.org/10.1128/aac.38.8.1864

Takashita, E., Hongo, S., Matsuzaki, Y., Noda, T., Furukawa, T., Sugawara, K., ... Shimotai, Y. (2010). Role of the CM2 Protein in the Influenza C Virus Replication Cycle. Journal of Virology, 85(3), 1322-1329. https://doi.org/10.1128/jvi.01367-10

Tan, S. Y., \& Tatsumura, Y. (2015). Alexander Fleming (1881-1955): Discoverer of penicillin. Singapore Medical Journal, 56(7), 366-367. https://doi.org/10.11622/smedj.2015105

Taubenberger, J. K., \& Morens, D. M. (2006). Taubenberger_EmergInfectDis_2006, 12(1), 1522. https://doi.org/10.3201/eid1201.050979

Taubenberger, J. K., \& Morens, D. M. (2008). The pathology of influenza virus infections. Annual Review of Pathology, 3, 499-522. https://doi.org/10.1146/annurev.pathmechdis.3.121806.154316

Tolins, M., Ruchirawat, M., \& Landrigan, P. (2014). The developmental neurotoxicity of arsenic: Cognitive and behavioral consequences of early life exposure. Annals of Global 
Health, 80(4), 303-314. https://doi.org/10.1016/j.aogh.2014.09.005

Tournel, G., Houssaye, C., Humbert, L., Dhorne, C., Gnemmi, V., Bécart-Robert, A., ... Lhermitte, M. (2011). Acute Arsenic Poisoning: Clinical, Toxicological, Histopathological, and Forensic Features. Journal of Forensic Sciences, 56(SUPPL. 1). https://doi.org/10.1111/j.1556-4029.2010.01581.x

Tyler, C. R., \& Allan, A. M. (2014). The Effects of Arsenic Exposure on Neurological and Cognitive Dysfunction in Human and Rodent Studies: A Review. Current Environmental Health Reports, 1(2), 132-147. https://doi.org/10.1007/s40572-014-0012-1

Uddin, R., \& Huda, N. H. (2011). Arsenic poisoning in Bangladesh. Oman Medical Journal, 26(3), 207. https://doi.org/10.5001/omj.2011.51

van de Sandt, C. E., Dou, Y. Y., Vogelzang-van Trierum, S. E., Westgeest, K. B., Pronk, M. R., Osterhaus, A. D. M. E., ... Hillaire, M. L. B. (2015). Influenza B virus-specific CD8+ Tlymphocytes strongly cross-react with viruses of the opposing influenza B lineage. Journal of General Virology, 96(8), 2061-2073. https://doi.org/10.1099/vir.0.000156

Van Halem, D., Bakker, S. A., Amy, G. L., \& Van Dijk, J. C. (2009). Arsenic in drinking water: A worldwide water quality concern for water supply companies. Drinking Water Engineering and Science, 2(1), 29-34. https://doi.org/10.5194/dwes-2-29-2009

Vanleuven, J. T., Ridenhour, B. J., Gonzalez, A. J., Miller, R., \& Miura, T. A. (2017). Lung epithelial cells have virus-specific and shared gene expression responses to infection by diverse respiratory viruses, $1-18$. https://doi.org/10.1371/journal.pone.0178408

Vareille, M., Kieninger, E., Edwards, M. R., \& Regamey, N. (2011a). The airway epithelium: Soldier in the fight against respiratory viruses. Clinical Microbiology Reviews, 24(1), 210229. https://doi.org/10.1128/CMR.00014-10 
Vareille, M., Kieninger, E., Edwards, M. R., \& Regamey, N. (2011b). The airway epithelium: Soldier in the fight against respiratory viruses. Clinical Microbiology Reviews, 24(1), 210229. https://doi.org/10.1128/CMR.00014-10

Varki, N. M., \& Varki, A. (2007). Diversity in cell surface sialic acid presentations: Implications for biology and disease. Laboratory Investigation, 87(9), 851-857. https://doi.org/10.1038/labinvest.3700656

Verhelst, J., Parthoens, E., Schepens, B., Fiers, W., \& Saelens, X. (2012). Interferon-Inducible Protein Mx1 Inhibits Influenza Virus by Interfering with Functional Viral Ribonucleoprotein Complex Assembly. Journal of Virology, 86(24), 13445-13455. https://doi.org/10.1128/jvi.01682-12

Virus, O. F. I. (1938). Serological races, 293-314.

Wade, T. J., Xia, Y., Mumford, J., Wu, K., Le, X. C., Sams, E., \& Sanders, W. E. (2015). Cardiovascular disease and arsenic exposure in Inner Mongolia, China: A case control study. Environmental Health: A Global Access Science Source, 14(1), 1-10. https://doi.org/10.1186/s12940-015-0022-y

Wang, M., \& Veit, M. (2016). Hemagglutinin-esterase-fusion (HEF) protein of influenza C virus. Protein and Cell, 7(1), 28-45. https://doi.org/10.1007/s13238-015-0193-x

Wang, Q. Q., Wang, C., Jiang, Y. H., Khairul, I., \& Naranmandura, H. (2017). Metabolism, toxicity and anticancer activities of arsenic compounds. Oncotarget, 8(14), 23905-23926. https://doi.org/10.18632/oncotarget.14733

Warne, R., Baldwin, D. M., Muiru, G., Bussiere, D. E., Ma, X., Xie, L., ... Zaror, I. (2017). Structural basis for therapeutic inhibition of influenza A polymerase PB2 subunit. Scientific Reports, 7(1), 1-8. https://doi.org/10.1038/s41598-017-09538-x 
Watanabe, T., Watanabe, S., \& Kawaoka, Y. (2010). Cellular networks involved in the influenza virus life cycle. Cell Host and Microbe, 7(6), 427-439. https://doi.org/10.1016/j.chom.2010.05.008

Webster, R. G., \& Govorkova, E. A. (2014). Continuing challenges in influenza. Annals of the New York Academy of Sciences, 1323(1), 115-139. https://doi.org/10.1111/nyas. 12462

Wei, C., Boyington, J. C., Dai, K., Houser, K. V, Melissa, B., Kong, W., ... Nabel, G. J. (2011). Cross-Neutralization of 1918 and 2009 Influenza Viruses: Role of Glycan in Viral Evolution and Vaccine Design. Science Translational Medicine, 2(24), 1-16. https://doi.org/10.1126/scitranslmed.3000799.Cross-Neutralization

Wei, S., Zhang, H., \& Tao, S. (2019). A review of arsenic exposure and lung cancer. Toxicology Research. https://doi.org/10.1039/c8tx00298c

Weir, J. P., \& Gruber, M. F. (2016). An overview of the regulation of influenza vaccines in the United States. Influenza and Other Respiratory Viruses, 10(5), 354-360. https://doi.org/10.1111/irv.12383

Whitley, R. J., \& Monto, A. S. (2006). Prevention and Treatment of Influenza in High-Risk Groups: Children, Pregnant Women, Immunocompromised Hosts, and Nursing Home Residents. The Journal of Infectious Diseases, 194(s2), S133-S138. https://doi.org/10.1086/507548

Whitsett, J. A. (2018). Airway epithelial differentiation and mucociliary clearance. Annals of the American Thoracic Society, 15, S143-S148. https://doi.org/10.1513/AnnalsATS.201802$128 \mathrm{AW}$

Who. (2011). Manual for the laboratory diagnosis and virological surveillance of influenza. World Health Organization 2011, 153. 
Wollina, U. (2016). Arsenic and skin cancer - case report with chemoprevention. Our Dermatology Online, 7(2), 172-175. https://doi.org/10.7241/ourd.20162.46

Wong, S. S. Y., \& Yuen, K. (2006). Avian Influenza Virus Infections in Humans. Chest, 129(1), 156-168. https://doi.org/10.1378/chest.129.1.156

Wu, D.-D., Lau, A. T. Y., Yu, F.-Y., Cai, N.-L., Dai, L.-J., Ok Kim, M., ... Xu, Y.-M. (2017). Extracellular signal-regulated kinase 8-mediated NF- $\mathrm{kB}$ activation increases sensitivity of human lung cancer cells to arsenic trioxide. Oncotarget, 8(30), 49144-49155. https://doi.org/10.18632/oncotarget.17100

Wu, X., Wu, X., Sun, Q., Zhang, C., Yang, S. Y., Li, L., \& Jia, Z. (2017). Progress of small molecular inhibitors in the development of anti-influenza virus agents. Theranostics, 7(4), 826-845. https://doi.org/10.7150/thno.17071

Xu, R., McBride, R., Nycholat, C. M., Paulson, J. C., \& Wilson, I. A. (2011). Structural Characterization of the Hemagglutinin Receptor Specificity from the 2009 H1N1 Influenza Pandemic. Journal of Virology, 86(2), 982-990. https://doi.org/10.1128/jvi.06322-11

Yapijakis, C. (2009). Hippocrates of Kos, the father of clinical medicine, and asclepiades of Bithynia, the father of molecular medicine. In Vivo, 23(4), 507-514.

Yoshida, H., Sakoda, Y., Endo, M., Motoshima, M., \& Yoshino, F. (2011). Evaluation of the Reverse Transcription Loop-Mediated Isothermal Amplification ( RT-LAMP) as a Screening Method for the Detection of Influenza Viruses in the Fecal Materials of Water Birds.

Yu, G., Sun, D., \& Zheng, Y. (2007). Health effects of exposure to natural arsenic in groundwater and coal in China: An overview of occurrence. Environmental Health Perspectives, 115(4), 636-642. https://doi.org/10.1289/ehp.9268 
Zamarin, D., Ortigoza, M. B., \& Palese, P. (2006). Influenza A Virus PB1-F2 Protein Contributes to Viral Pathogenesis in Mice. Journal of Virology, 80(16), 7976-7983. https://doi.org/10.1128/jvi.00415-06

Zeinvand-Lorestani, M., Kalantari, H., Khodayar, M. J., Teimoori, A., Saki, N., Ahangarpour, A., ... Alboghobeish, S. (2018). Autophagy upregulation as a possible mechanism of arsenic induced diabetes. Scientific Reports, 8(1), 1-15. https://doi.org/10.1038/s41598018-30439-0

Zhang, J.-M., \& Jianxiong, A. (2009). Cytokines, Inflammation and Pain. Int Anesthesiol Clin., 69(2), 482-489. https://doi.org/10.1097/AIA.0b013e318034194e.Cytokines

Zhang, X., Tang, N., Hadden, T. J., \& Rishi, A. K. (2011). Akt, FoxO and regulation of apoptosis. Biochimica et Biophysica Acta - Molecular Cell Research, 1813(11), 1978-1986. https://doi.org/10.1016/j.bbamcr.2011.03.010

Zhao, F., \& Klimecki, W. T. (2015). Culture conditions profoundly impact phenotype in BEAS2B, a human pulmonary epithelial model. Journal of Applied Toxicology, 35(8), 945-951. https://doi.org/10.1002/jat.3094

Zhao, F., Malm, S. W., Hinchman, A. N., Li, H., Beeks, C. G., \& Klimecki, W. T. (2014). Arsenite-induced pseudo-hypoxia results in loss of anchorage-dependent growth in BEAS2B pulmonary epithelial cells. PLoS ONE, 9(12), 1-16. https://doi.org/10.1371/journal.pone.0114549

Zhong, M., Huang, Z., Wang, L., Lin, Z., Cao, Z., Li, X., ... Ma, X. (2018). Malignant Transformation of Human Bronchial Epithelial Cells Induced by Arsenic through STAT3/miR-301a/SMAD4 Loop. Scientific Reports, 8(1), 1-12. https://doi.org/10.1038/s41598-018-31516-0 